\title{
The phylogenetic nomenclature of ornithischian dinosaurs
}

\author{
Daniel Madzia ${ }^{\text {Corresp., } 1}$, Victoria M. Arbour ${ }^{2}$, Clint A. Boyd ${ }^{3}$, Andrew A. Farke ${ }^{4}$, Penélope Cruzado-Caballero ${ }^{5,6,7,8}$, \\ David C Evans ${ }^{9}$ \\ ${ }^{1}$ Department of Evolutionary Paleobiology, Institute of Paleobiology, Polish Academy of Sciences, Warsaw, Poland \\ 2 Department of Knowledge, Royal BC Museum, Victoria, BC, Canada \\ 3 North Dakota Geological Survey, Bismarck, ND, USA \\ 4 Raymond M. Alf Museum of Paleontology at The Webb Schools, Claremont, CA, USA \\ 5 Área de Paleontología, Departamento de Biología Animal, Edafología y Geología, Universidad de La Laguna, Santa Cruz de Tenerife, Spain \\ 6 Instituto de Investigación en Paleobiología y Geología (IIPG), Universidad Nacional de Río Negro, Río Negro, Argentina \\ 7 Instituto de Investigación en Paleobiología y Geología (IIPG), Consejo Nacional de Investigaciones Científicas y Tecnológicas (CONICET), Río Negro, \\ Argentina \\ ${ }^{8}$ Grupo Aragosaurus-IUCA, Área de Paleontología, Departamento de Ciencias de la Tierra, Universidad de Zaragoza, Zaragoza, Spain \\ 9 Department of Natural History, Royal Ontario Museum, Toronto, ON, Canada \\ Corresponding Author: Daniel Madzia \\ Email address: daniel.madzia@gmail.com
}

Ornithischians form a large clade of globally distributed Mesozoic dinosaurs, and one of their three major radiations. Throughout their evolutionary history, exceeding 134 million years, ornithischians evolved considerable morphological disparity, expressed especially through the cranial and osteodermal features of their most distinguishable representatives. The nearly two-century-long research history on ornithischians has resulted in the recognition of numerous diverse lineages, many of which have been named. Following the formative publications establishing the theoretical foundation of phylogenetic nomenclature throughout the 1980s and 1990s, many of the proposed names of ornithischian clades were provided with phylogenetic definitions. Some of these definitions have proven useful and have not been changed, beyond the way they were formulated, since their introduction. Some names, however, have multiple definitions, making their application ambiguous. Recent implementation of the International Code of Phylogenetic Nomenclature (ICPN, or PhyloCode) offers the opportunity to explore the utility of previously proposed definitions of established taxon names. Since the Articles of the ICPN are not to be applied retroactively, all phylogenetic definitions published prior to its implementation remain informal (and ineffective) in the light of the Code. Here, we revise the nomenclature of ornithischian dinosaur clades; we revisit 76 preexisting ornithischian clade names, review their recent and historical use, and formally establish their phylogenetic definitions. Additionally, we introduce five new clade names; two for robustly supported clades of later-diverging hadrosaurids and ceratopsians, one uniting 
heterodontosaurids and genasaurs, and two for clades of nodosaurids. Our study marks a key step towards a formal phylogenetic nomenclature of ornithischian dinosaurs. 


\section{The phylogenetic nomenclature of ornithischian dinosaurs}

2

3 Daniel Madzia ${ }^{1,}$, Victoria M. Arbour ${ }^{2}$, Clint A. Boyd ${ }^{3}$, Andrew A. Farke ${ }^{4}$, Penélope Cruzado-

4 Caballero $^{5,6,7,8}$, David C. Evans ${ }^{9}$

5

6

7

8

\section{${ }^{1}$ Department of Evolutionary Paleobiology, Institute of Paleobiology, Polish Academy of Sciences, Warsaw, Poland} ${ }^{2}$ Department of Knowledge, Royal BC Museum, Victoria, BC, Canada

${ }^{3}$ North Dakota Geological Survey, Bismarck, ND, USA

${ }^{4}$ Raymond M. Alf Museum of Paleontology at The Webb Schools, Claremont, CA, USA

${ }^{5}$ Area de Paleontología, Departamento de Biología Animal, Edafología y Geología, Universidad de La Laguna, Santa Cruz de Tenerife, Spain

${ }^{6}$ Instituto de Investigación en Paleobiología y Geología (IIPG), Universidad Nacional de Río Negro, Río Negro, Argentina

${ }^{7}$ Instituto de Investigación en Paleobiología y Geología (IIPG), Consejo Nacional de Investigaciones Científicas y Tecnológicas (CONICET), Río Negro, Argentina

${ }^{8}$ Grupo Aragosaurus-IUCA, Área de Paleontología, Departamento de Ciencias de la Tierra, Universidad de Zaragoza, Zaragoza, Spain

${ }^{9}$ Department of Natural History, Royal Ontario Museum, Toronto, ON, Canada

*Corresponding author: daniel.madzia@gmail.com

Abstract. Ornithischians form a large clade of globally distributed Mesozoic dinosaurs, and one of their three major radiations. Throughout their evolutionary history, exceeding 134 million years, ornithischians evolved considerable morphological disparity, expressed especially through the cranial and osteodermal features of their most distinguishable representatives. The nearly two-century-long research history on ornithischians has resulted in the recognition of numerous diverse lineages, many of which have been named. Following the formative publications establishing the theoretical foundation of phylogenetic nomenclature throughout the 1980s and 1990s, many of the proposed names of ornithischian clades were provided with phylogenetic definitions. Some of these definitions have proven useful and have not been changed, beyond the way they were formulated, since their introduction. Some names, however, have multiple definitions, making their application ambiguous. Recent implementation of the International Code of Phylogenetic Nomenclature (ICPN, or PhyloCode) offers the opportunity to explore the 
utility of previously proposed definitions of established taxon names. Since the Articles of the $I C P N$ are not to be applied retroactively, all phylogenetic definitions published prior to its implementation remain informal (and ineffective) in the light of the Code. Here, we revise the nomenclature of ornithischian dinosaur clades; we revisit 76 preexisting ornithischian clade names, review their recent and historical use, and formally establish their phylogenetic definitions. Additionally, we introduce five new clade names; two for robustly supported clades of later-diverging hadrosaurids and ceratopsians, one uniting heterodontosaurids and genasaurs, and two for clades of nodosaurids. Our study marks a key step towards a formal phylogenetic nomenclature of ornithischian dinosaurs.

Keywords: Phylogenetic nomenclature, phylogenetic definition, PhyloCode, International Code of Phylogenetic Nomenclature, Ornithischia, Dinosauria.

\section{Introduction}

The ornithischian, or 'bird-hipped', dinosaurs were a species-rich clade of Mesozoic archosaurs that first appeared in the Triassic (e.g., Langer \& Ferigolo, 2013; Cabreira et al., 2016; Pacheco et al., 2019; Desojo et al., 2020; Müller \& Garcia, 2020) or the earliest Jurassic (Agnolín \& Rozadilla 2018; Baron, 2019) and died out during the Cretaceous/Paleogene extinction event (e.g., Brusatte et al., 2015). Throughout their $>134$ million-year-long evolutionary history, ornithischians achieved global distribution (Weishampel et al., 2004; Boyd, 2015), evolved considerable taxic diversity (Tennant et al., 2018), and an apparent morphological disparity, expressed through their markedly different body sizes (Benson et al., 2018) and especially the 'exaggerated' structures of the crania and osteodermal armor of some of their most distinctive members (e.g., Brown, 2017; Stubbs et al., 2019).

Here, we provide a nomenclatural revision of ornithischian dinosaur clades. Following the pivotal, early formative publications establishing the theoretical foundation of the phylogenetic nomenclature in the 1980s and early 1990s (e.g., Ghiselin, 1984; Gauthier, 1986; Rowe, 1987; de Queiroz, 1988; Estes et al., 1988; Gauthier et al., 1988; de Queiroz and Gauthier, 1990, 1992, 1994), many names of the ornithischian clades were provided phylogenetic definitions (e.g., Padian \& May, 1993; Currie \& Padian, 1997; Sereno, 1998; Sereno, 1999), some of which have 
64 proven useful and have not been changed, beyond the way they were formulated, since their 65 introduction.

66 The implementation of the International Code of Phylogenetic Nomenclature, or the PhyloCode

67 (de Queiroz \& Cantino, 2020), an evolution-based system for naming organisms, hereafter 68 abbreviated and referred to as $\operatorname{ICPN}$ (accessible at http://phylonames.org/code/), and parallel publication of Phylonyms: A Companion to the PhyloCode (de Queiroz et al., 2020), offers the opportunity to consider the utility of previously proposed phylogenetic definitions of established taxon names and, in appropriate cases, formalize their use, as specified by the Articles of the $I C P N$.

Recent studies have thoroughly assessed the use of clade names applied to some ornithischian lineages, mostly early-diverging neornithischians and ornithopods (Boyd, 2015; Madzia et al., 2018; Herne et al., 2019; Madzia et al., 2020). However, the Articles of the $I C P N$ are not to be applied retroactively (ICPN: Preamble 6, see also Art. 7.1). As such, all these efforts remain informal and ineffective in the light of the Code.

We formalize some of the nomenclatural acts of previous studies and introduce phylogenetic definitions for 81 names of ornithischian dinosaur clades. Specifically, we provide formal phylogenetic definitions for the following 76 preexisting taxon names: Ankylopollexia, Ankylosauria, Ankylosauridae, Ankylosaurinae, Ankylosaurini, Aralosaurini, Brachylophosaurini, Camptosauridae, Centrosaurinae, Centrosaurini, Cerapoda, Ceratopsia, Ceratopsidae, Ceratopsoidea, Chaoyangsauridae, Chasmosaurinae, Clypeodonta, Coronosauria, Dryomorpha, Dryosauridae, Edmontosaurini, Elasmaria, Eucentrosaura, Euhadrosauria, Euiguanodontia, Euornithopoda, Eurypoda, Genasauria, Hadrosauridae, Hadrosauriformes, Hadrosaurinae, Hadrosauroidea, Hadrosauromorpha, Heterodontosauridae, Huayangosauridae, Hypsilophodontia, Hypsilophodontidae, Iguanodontia, Iguanodontidae, Jeholosauridae, Kritosaurini, Lambeosaurinae, Lambeosaurini, Leptoceratopsidae, Marginocephalia, Nasutoceratopsini, Neoceratopsia, Neoiguanodontia, Neornithischia, Nodosauridae, Nodosaurinae, Ornithischia, Ornithopoda, Orodrominae, Pachycephalosauria, Pachycephalosauridae, Pachycephalosaurinae, Pachycephalosaurini, Pachyrhinosaurini, Pachyrostra, Parasaurolophini, Polacanthinae, Protoceratopsidae, Rhabdodontidae, Rhabdodontomorpha, Saurolophinae, Saurolophini, Shamosaurinae, Stegosauria, Stegosauridae, Styracosterna, Thescelosauridae, Thescelosaurinae, Thyreophora, 
Triceratopsini, and Tsintaosaurini. These names cover all major ornithischian clades and the vast majority of their subclades for which taxon names were used and defined in the past. Additionally, we introduce five new clade names: Corythosauria, for the node uniting lambeosaurin and parasaurolophin lambeosaurines, Euceratopsia, for the node uniting leptoceratopsid and coronosaur ceratopsians, Saphornithischia, for the node uniting heterodontosaurids and genasaurs, and Panoplosaurini and Struthiosaurini for clades of laterdiverging nodosaurids.

\section{Institutional abbreviations}

CMN, Canadian Museumof Nature, Ottawa, Canada; CPC, Colección Paleontológica de Coahuila, Museo del Desierto, Saltillo, Mexico; GPDM, Great Plains Dinosaur Museum, Malta, Montana, USA; MOR, Museum of the Rockies, Bozeman, Montana, USA; PASAC, Paleontological Association of Sabinas, Coahuila, Mexico; ROM, Royal Ontario Museum, Department of Natural History, Toronto, Canada; UTEP, Centennial Museum and Chihuahuan Desert Gardens, University of Texas at El Paso, Texas, USA; ZPAL, Institute of Paleobiology, Polish Academy of Sciences, Warsaw, Poland.

\section{Methods}

\section{Protocol}

In order to be formally established under the $I C P N$, clade names must comply especially with the provisions of Articles 7 and 9-11 of the Code (ICPN: Art. 7.2d). These Articles are fully followed here. The entries, provided in 'Phylogenetic nomenclature of ornithischian clades' below, partly follow the scheme used in Phylonyms (de Queiroz et al., 2020); they include the following sub-sections: 'Definition', 'Reference phylogeny', 'Composition', 'Synonyms', and 'Comments'. The sub-sections 'Diagnostic apomorphies' and 'Etymology', as used in Phylonyms, have been omitted. Note that detailed discussion of apomorphies is not strictly required by the Code, and inclusion of a reference phylogeny alone is sufficient (ICPN: Art. 9.13). Recent assessments of the phylogenetic relationships of numerous taxa, particularly those nested near the basal neornithischian-ornithopod transition, but also within some major clades, such as ornithopods, currently provide conflicting results (e.g., Norman, 2015; Han et al., 2018; Madzia et al., 2018; Andrzejewski et al., 2019; Herne et al., 2019; Párraga \& Prieto-Márquez, 
126 2019; Dieudonné et al., 2020; Yang et al., 2020; Barta \& Norell, 2021; Černý et al., 2021). It is

127 extremely difficult, and perhaps impossible at the moment, to list unambiguous diagnostic

128 apomorphies for many clades that have long been associated with widely-used names, and

129 detailed discussion would be far beyond the scope of the paper. Instead, emphasis was placed on

130 using definitions that are reflective of all currently inferred phylogenies. In turn, 'Etymology'

131 was omitted because all but five of the clade names that are established in the present study are

132 preexisting (Art. 6.2 of the ICPN). The only reason for discussing the etymological origin of

133 taxon names would be to provide arguments for the inclusion of certain internal specifiers (e.g.,

134 within the context of Art. 11.10 of the $I C P N)$. With that respect, relevant comments are provided

135 in the 'Comments' sub-section of the name entries. The five new clade names introduced in the

136 present study are provided with their etymologies. Additionally, owing to the fact that the

137 phylogenetic relationships of ornithischian dinosaurs are intensively researched, each clade name

138 entry could be supplemented with numerous reference phylogenies. Rather than list all of the

139 relevant phylogeny reconstructions available, we decided to refer to a subset of the more recent

140 tree topologies that justify the 'conversion' of the taxon name in accordance with the ICPN.

141 We have not followed any strict approach while selecting primary reference phylogenies. Instead

142 of providing references to studies that represent, for example, the most recent iterations of some

143 datasets, we preferred to refer to studies that we have been either directly involved in, and are

144 therefore familiar with the original data used for phylogeny inference, or consider to cover

145 relevant data sampling.

146 With respect to the clade 'Composition', we list only those subtaxa that are included in the

147 primary reference phylogeny. It is essential to realize that some of the clades for which names

148 are provided have insufficiently explored origins and their basal branching is expressed through

149 polytomies (this applies especially, but not exclusively, to non-hadrosaurid ornithopod

150 subclades). In such cases, the actual extent may not be certain and some of the taxa listed in the

151 'Composition' subsection may in fact fall outside the clades. Note also that some of the selected

152 primary reference phylogenies do not show the placements of all taxa used as specifiers in the

153 definitions of the names to be defined. In such cases, the phylogenetic positions of these

154 specifiers are discussed in the 'Comments'.

155 We also realize that the list of taxon names provided in 'Synonyms' is not exhaustive and does

156 not list all historically used approximate synonyms. When discussing names that may be 
157 considered synonymous with those whose application is preferred here, we have focused

158 especially on those names that have been used for the same or very similar contents in recent years, or those that have been used interchangeably with those that we define (e.g.,

160 Iguanodontidae and Iguanodontoidea, Thescelosauridae and Parksosauridae). Therefore, the 161 names that have not been in use for a long time were mostly omitted.

162 Further, Article 8.1 of the $I C P N$ states that, "[i]n order for a name to be established under [the $163[C P N]$, the name and other required information must be submitted to the registration database 164 for phylogenetically defined names (see Art. 22.2). A name may be submitted to the database prior to acceptance for publication, but it is given only a temporary registration number at that time. The registration number will become permanent after the author notifies the database that the paper or book in which the name will appear has been published, provides a full reference to the publication, and confirms that the definition in the database is identical to that in the publication". We have therefore registered all names, whose phylogenetic definitions are established in the present study, to the database of phylogenetically defined names, the RegNum (ICPN: Art. 22; Appendix A), and obtained registration numbers that are included in the clade name entries.

173 Finally, we follow the $I C P N$ in that all scientific names are italicized (ICPN: Recommendation 174 6.1A.) and that names are attributed to the earliest author(s) to spell them rather than according to the Principle of Coordination (ICPN: Note 9.15A.3).

\section{Phylogenetic definitions}

The names of ornithischian clades are defined using the following two types of definitions: (a) minimum-clade definition, known previously as 'node-based' definition (ICPN: Art. 9.5) and (b) maximum-clade definition, known previously as 'branch-based' or 'stem-based' definition (ICPN: Art. 9.6). We refer to the aforementioned Articles of the ICPN for details.

Adopted conventions for abbreviated definitions. We abbreviate the definitions using the following conventions (as per Notes 9.4.1 and 11.12.1 of the $I C P N$ ): $\max =$ the largest; $\min =$ the smallest; $\nabla=$ clade; ()$=$ containing; $\&=$ and; $\vee=$ or; $\sim=$ but not (in trivial maximum-clade definitions) or it does not (while using a qualifying clause) $\mid=$ on the condition that. See also Note 9.6.2 of the ICPN for explanation of differences between the use of ' $\&$ ' and ' $\mathrm{V}$ ' in the 
188

definitions. Additionally, we apply the set theory symbols $\in$, that means "belongs to", and $\notin$, meaning "not element of", to indicate that a name is applied within or outside another clade, respectively (see Euornithopoda, Jeholosauridae, Orodrominae, and Polacanthinae for some examples).

Selection of specifiers. Specifiers are selected following Art. 11 of the $I C P N$. Numerous names pertaining to ornithischian clades have been informally defined in the past and these definitions can still be considered applicable. We have attempted to formalize most of these definitions, providing only the changes that were necessary to reflect all currently inferred phylogenies and to comply with the Articles of the ICPN. However, in some cases we have decided to replace certain specifiers with taxa that we consider to be more appropriate candidates. For example, we have replaced Parasaurolophus walkeri Parks, 1922 in some definitions with Iguanodon bernissartensis Boulenger in Beneden, 1881 (designated as the type species of Iguanodon Mantell, 1825 by the International Commission on Zoological Nomenclature [ICZN, 2000]), provided that this taxon has always been considered part of the clade (when selected as an internal specifier) or outside the clade (when selected as an external specifier) whose name is being defined. I. bernissartensis is known based on multiple complete or near-complete individuals of different ontogenetic stages and is being extensively researched (e.g., Norman, 1980; Verdú et al., 2017). It has also been frequently used as the specifier in previous, informal phylogenetic definitions, and was recently included as the internal specifier of Dinosauria (Langer et al., 2020). It is further essential to note that some taxa had to be used as internal specifiers despite their suggested dubious taxonomic status. For example, Ceratops montanus Marsh, 1888 is the name-bearer of Ceratopsia, Ceratopsoidea, Ceratopsidae, and Ceratopsinae (the last name is not converted to a clade name in the present study). At the same time, however, the taxon is generally considered to lack diagnostic features and is commonly treated a nomen dubium (e.g., Dodson et al., 2004; Mallon et al., 2016). Following Article 11.10 of the ICPN (which specifies that "[w]hen a clade name is converted from a preexisting name that is typified under a rank-based code or is a new or converted name derived from the stem of a typified name, the definition of the clade name must use the type species of that preexisting typified name or of the genus name from which it is derived (or the type specimen of that species) as an internal 
218 specifier.”), Ceratops montanus must be the internal specifier (or among the internal specifiers)

219 in the definitions of the names in question.

220

\section{Phylogenetic nomenclature of ornithischian clades}

222 For the sake of clarity, all clade names are provided in alphabetical order. The definitions are 223 summarized in Table 1. The extent of all clade names is further depicted on Fig. 1. that shows 224 the relationships of taxa included in the present study as specifiers (both, internal as well as 225 external) and additionally on Figs 2, 3, and 4 that represent selected ornithischian-wide 226 phylogenies published within recent years: Madzia et al. (2018: Fig. 4B), Dieudonné et al. (2020:

227 Figs 1 and 2), and Yang et al. (2020: Fig. 12).

228

229

[Table 1 here]

230

231

232

Ankylopollexia Sereno, 1986 [converted clade name]

233

Registration number: 585

Definition. The smallest clade containing Camptosaurus dispar (Marsh, 1879) and Iguanodon bernissartensis Boulenger in Beneden, 1881. This is a minimum-clade definition. Abbreviated definition: $\min \nabla$ (Camptosaurus dispar [Marsh, 1879] \& Iguanodon bernissartensis Boulenger in Beneden, 1881).

Reference phylogeny. Figure 12 of Madzia et al. (2020) is treated here as the primary reference phylogeny. Additional reference phylogenies include Figure 3 of Madzia et al. (2018), Figure 20 of Verdú et al. (2018), Figure 11 of McDonald et al. (2021), Figure 11 of Santos-Cubedo et al. (2021), and Figure 9 of Verdú et al. (2020).

Composition. The clade Ankylopollexia comprises Camptosaurus dispar and members of the clade Styracosterna.

Synonyms. No other taxon names are currently in use for the same or approximate clade.

Comments. The name Ankylopollexia has been (informally) defined before by Sereno (1998: 62) who applied the minimum-clade definition and used Camptosaurus and Parasaurolophus as the internal specifiers. Since the name has traditionally been used in the exact sense, we apply it to the same clade, but prefer to use Iguanodon bernissartensis as the second internal specifier rather than P. walkeri because the name Ankylopollexia was formed after the stiff cone-shaped thumb 
249 that characterizes Iguanodon-grade ornithopods. The inclusion of a different internal specifier

250 does not change the extent of Ankylopollexia under any of the published phylogeny inferences.

251 Also, even though the name derives from an apomorphy, it was never used for an apomorphy-

252 based clade.

253

254 Ankylosauria Osborn, 1923 [converted clade name]

255 Registration number: 588

256 Definition. The largest clade containing Ankylosaurus magniventris Brown, 1908 but not

257 Stegosaurus stenops Marsh, 1887. This is a maximum-clade definition. Abbreviated definition:

$258 \max \nabla$ (Ankylosaurus magniventris Brown, 1908 Stegosaurus stenops Marsh, 1887).

259 Reference phylogeny. Figure 11 of Arbour and Currie (2016) is treated here as the primary 260 reference phylogeny. Additional reference phylogenies include Figure 3 of Thompson et al.

261 (2012), Figure 1 of Arbour et al. (2016), Figure 3 of Brown et al. (2017), and Figure 26 of

262 Wiersma \& Irmis (2018).

263 Composition. Under the primary reference phylogeny, Ankylosauria comprises Minmi sp. (=

264 Kunbarrasaurus ieversi), Mymoorapelta maysi, and members of the clades Ankylosauridae and 265 Nodosauridae.

266 Synonyms. The name Ankylosauromorpha Carpenter, 2001 has been recently used under an

267 alternative systematic scheme for the same branch as Ankylosauria, as defined herein (Norman,

268 2021; see 'Discussion'). No other taxon names are currently in use for the same or approximate 269 clade.

270 Comments. The name Ankylosauria has been (informally) defined before (Carpenter, 1997;

271 Sereno, 1998; Sereno, 2005). These definitions were maximum-clade and used Ankylosaurus

272 (Carpenter, 1997; Sereno, 1998) or Ankylosaurus magniventris (Sereno, 2005) as the internal

273 specifier and Stegosaurus (Carpenter, 1997; Sereno, 1998) or Stegosaurus stenops (Sereno,

274 2005) as the external specifier. Since Ankylosauria has been 'traditionally' used in this sense

275 (though, see also 'Discussion'), we formalize this definition. Note that Norman (2021) recently

276 provided two phylogenetic definitions for Ankylosauria, a maximum-clade and a minimum-

277 clade. In the maximum-clade definition Norman (2021) used Euoplocephalus and Edmontonia as

278 the internal specifiers and Scelidosaurus as the external specifier, while in the minimum-clade

279 definition the use of the name was anchored on Euoplocephalus and Edmontonia. See

Peer] reviewing PDF | (2021:04:60693:1:1:NEW 8 Sep 2021) 
280 'Discussion' for additional comments. Note that the external specifier Stegosaurus stenops is not 281 included in the primary reference phylogeny. From the taxa analyzed by Arbour \& Currie 282 (2016), S. stenops is most closely related to Huayangosaurus taibaii (see, e.g., Maidment et al., 283 2020).

Ankylosauridae Brown, 1908 [converted clade name]

286 Registration number: 589

287 Definition. The largest clade containing Ankylosaurus magniventris Brown, 1908 but not 288 Nodosaurus textilis Marsh, 1889. This is a maximum-clade definition. Abbreviated definition: $289 \max \nabla$ (Ankylosaurus magniventris Brown, 1908 Nodosaurus textilis Marsh, 1889).

290 Reference phylogeny. Figure 11 of Arbour \& Currie (2016) is treated here as the primary 291 reference phylogeny. Additional reference phylogenies include Figure 3 of Thompson et al.

292 (2012), Figure 1 of Arbour et al. (2016), Figure 3 of Brown et al. (2017), Figure 26 of Wiersma 293 \& Irmis (2018), and Figure 9 of Zheng et al. (2018).

294 Composition. Under the primary reference phylogeny, Ankylosauridae comprises Ahshislepelta 295 minor, Aletopelta coombsi, Cedarpelta bilbeyhallorum, Chuanqilong chaoyangensis, Gastonia

296 burgei, Liaoningosaurus paradoxus, and members of the clades Shamosaurinae and 297 Ankylosaurinae.

298 Synonyms. No other taxon names are currently in use for the same or approximate clade.

299 Comments. The name Ankylosauridae has been (informally) defined before by Sereno (1998, 300 2005) who applied a maximum-clade definition and used Ankylosaurus magniventris as the 301 internal specifier and Panoplosaurus mirus as the external specifier. Considering that 302 Ankylosauridae has been traditionally used as a sister taxon to Nodosauridae (see, e.g., 303 Thompson et al., 2012 for details), we use a definition that incorporates Nodosaurus textilis as 304 the external specifier. Note that $N$. textilis is not included in the primary reference phylogeny. 305 Both, A. magniventris and N. textilis were analyzed by, and their relationship is indicated in, 306 Rivera-Sylva et al. (2018a).

307 
310 Definition. The largest clade containing Ankylosaurus magniventris Brown, 1908 but not

311 Shamosaurus scutatus Tumanova, 1983. This is a maximum-clade definition. Abbreviated

312 definition: $\max \nabla$ (Ankylosaurus magniventris Brown, 1908 Shamosaurus scutatus Tumanova, 313 1983).

314 Reference phylogeny. Figure 11 of Arbour and Currie (2016) is treated here as the primary 315 reference phylogeny. Additional reference phylogenies include Figure 3 of Thompson et al.

316 (2012), Figure 1 of Arbour et al. (2016), Figure 8 of Arbour \& Evans (2017), Figure 26 of

317 Wiersma \& Irmis (2018), and Figure 9 of Zheng et al. (2018).

318 Composition. Under the primary reference phylogeny, Ankylosaurinae comprises Crichtonpelta

319 benxiensis, Pinacosaurus spp., Saichania chulsanensis, Tarchia kielanae, Tsagantegia

320 longicranialis, Zaraapelta nomadis, 'Zhejiangosaurus luoyangensis', and members of the clade 321 Ankylosaurini.

322 Synonyms. No other taxon names are currently in use for the same or approximate clade.

323 Comments. The name Ankylosaurinae was (informally) defined before (Sereno, 1998; Sereno,

324 2005; Vickaryous et al., 2004). All these definitions were maximum-clade and used

325 Ankylosaurus (Sereno, 1998) or Ankylosaurus magniventris (Sereno, 2005; Vickaryous et al.,

326 2004) as the internal specifiers and Minmi paravertebra and Shamosaurus scutatus (Sereno,

327 1998), Gargoyleosaurus parkpinorum, Minmi paravertebra, and Shamosaurus scutatus (Sereno,

328 2005) or only Shamosaurus scutatus (Vickaryous et al., 2004) as the external specifiers. Owing

329 to the dubious taxonomic status of ' $M$. paravertebra' (Arbour \& Currie, 2016) and non-

330 ankylosaurid affinities of G. parkpinorum (e.g., Arbour \& Currie, 2016; Rivera-Sylva et al.,

331 2018a; Wiersma \& Irmis, 2018; Zheng et al., 2018), we formalize the definition of Vickaryous et

332 al. (2004) in that we use a single external specifier (Shamosaurus scutatus).

333

334 Ankylosaurini Arbour \& Currie, 2016 [converted clade name]

335 Registration number: 592

336 Definition. The largest clade containing Ankylosaurus magniventris Brown, 1908 but not

337 Pinacosaurus grangeri Gilmore, 1933 and Saichania chulsanensis Maryańska, 1977. This is a

338 maximum-clade definition. Abbreviated definition: max $\nabla$ (Ankylosaurus magniventris Brown,

3391908 Pinacosaurus grangeri Gilmore, 1933 \& Saichania chulsanensis Maryańska 1977).

Peer] reviewing PDF | (2021:04:60693:1:1:NEW 8 Sep 2021) 
340 Reference phylogeny. Figure 11 of Arbour \& Currie (2016) is treated here as the primary

341 reference phylogeny. Additional reference phylogenies include Figure 1 of Arbour et al. (2016),

342 Figure 8 of Arbour \& Evans (2017), Figure 26 of Wiersma \& Irmis (2018), and Figure 9 of

343 Zheng et al. (2018).

344 Composition. Under the primary reference phylogeny, Ankylosaurini comprises Ankylosaurus

345 magniventris, Anodontosaurus lambei, Dyoplosaurus acutosquameus, Euoplocephalus tutus,

346 Nodocephalosaurus kirtlandensis, Scolosaurus cutleri, Talarurus plicatospineus, and Ziapelta

347 sanjuanensis.

348 Synonyms. No other taxon names are currently in use for the same or approximate clade.

349 Comments. The name Ankylosaurini was first (informally) defined by Arbour \& Currie (2016)

350 who applied the maximum-clade definition and used Ankylosaurus magniventris as the internal

351 specifier and Pinacosaurus grangeri and Saichania chulsanensis as the external specifiers. The

352 name was used for a clade that largely includes later-diverging North American ankylosaurines,

353 many of which were previously synonymized with Euoplocephalus tutus (Arbour \& Currie

354 2013), although under some topologies the name may be more restricted in its use (Thompson et

355 al., 2012).

356

357

358

Aralosaurini Prieto-Márquez et al., 2013 [converted clade name]

359

Registration number: 593

359

Definition. The largest clade containing Aralosaurus tuberiferus Rozhdestvensky, 1968 and

360

Canardia garonnensis Prieto-Márquez et al., 2013 but not Lambeosaurus lambei Parks, 1923,

361

Parasaurolophus walkeri Parks, 1922, and Tsintaosaurus spinorhinus Young, 1958. This is a

362

maximum-clade definition. Abbreviated definition: max $\nabla$ (Aralosaurus tuberiferus

363

Rozhdestvensky, 1968 \& Canardia garonnensis Prieto-Márquez et al., $2013 \sim$ Lambeosaurus

364

lambei Parks, 1923 \& Parasaurolophus walkeri Parks, 1922 \& Tsintaosaurus spinorhinus

365

Young, 1958).

366 Reference phylogeny. Figure 25 of Prieto-Márquez et al. (2013) is treated here as the primary

367

reference phylogeny. Additional reference phylogeny includes Figure 11 of McDonald et al.

368 (2021).

369 Composition. Under the primary reference phylogeny, Aralosaurini comprises Aralosaurus

370 tuberiferus and Canardia garonnensis. 
371 Synonyms. No other taxon names are currently in use for the same or approximate clade.

372 Comments. The name was first (informally) defined by Prieto-Márquez et al. (2013) who

373 applied the minimum-clade definition and used Aralosaurus tuberiferus and Canardia

374 garonnensis as the internal specifiers. Following such definition, however, Aralosaurini would

375 cover the entire lambeosaurine branch under some topologies that include both of the internal

376 specifiers (Kobayashi et al., 2019; Prieto-Márquez et al., 2019; Zhang et al., 2019; Gates et al.,

377 2021; Kobayashi et al., 2021; Longrich et al., 2021), or would even comprise the same contents

378 as Euhadrosauria (Ramírez-Velasco et al., 2021). Recently, however, McDonald et al. (2021)

379 inferred Aralosaurini as delimited by Prieto-Márquez et al. (2013). Therefore, we define the

380 name but make it inapplicable under a subset of recent phylogenies.

381

382 Brachylophosaurini Gates et al., 2011 [converted clade name]

383 Registration number: 594

384 Definition. The largest clade containing Brachylophosaurus canadensis Sternberg, 1953 but not

385 Edmontosaurus regalis Lambe, 1917, Hadrosaurus foulkii Leidy, 1858, Kritosaurus navajovius

386 Brown, 1910, and Saurolophus osborni Brown, 1912. This is a maximum-clade definition.

387 Abbreviated definition: $\max \nabla$ (Brachylophosaurus canadensis Sternberg, 1953

388 Edmontosaurus regalis Lambe, 1917 \& Hadrosaurus foulkii Leidy, 1858 \& Kritosaurus

389 navajovius Brown, 1910 \& Saurolophus osborni Brown, 1912).

390 Reference phylogeny. Figure 18 of Prieto-Márquez et al. (2020) is treated here as the primary

391 reference phylogeny. Additional reference phylogenies include Figure 5 of Kobayashi et al.

392 (2019), Figure 11 of Prieto-Márquez et al. (2019), Figure 9 of Zhang et al. (2019), Figure 5 of

393 Zhang et al. (2020), Figure 7 of Kobayashi et al. (2021), and Figure 10 of Longrich et al. (2021).

394 Composition. Under the primary reference phylogeny, Brachylophosaurini comprises

395 Acristavus gagslarsoni, Brachylophosaurus canadensis, Maiasaura peeblesorum, and

396 Probrachylophosaurus bergei (erroneously named 'Probrachylophosaurus canadensis' in the

397 primary reference phylogeny).

398 Synonyms. The name Maiasaurini Sereno, 2005 is an approximate synonym of

399 Brachylophosaurini. To our knowledge, the name was used only in two recent papers

400 (McFeeters et al., 2021a; McFeeters et al., 2021b) that attributed the name to Horner (1992).

401 However, this attribution was due to the adherence of the authors to the Principle of 
402 Coordination, as Horner (1992) used the name Maiasaurinae. Nevertheless, all recent

403 phylogenetic studies consistently use Brachylophosaurini (e.g., Freedman Fowler \& Horner, 404 2015; Cruzado-Caballero \& Powell, 2017; Xing et al., 2017; Kobayashi et al., 2019; Zhang et al., 405 2019; Prieto-Márquez et al., 2020; Zhang et al., 2020; Kobayashi et al., 2021; McDonald et al., 406 2021). No other taxon names are currently in use for the same or approximate clade.

407 Comments. The name Brachylophosaurini has been (informally) defined before (Gates et al., 408 2011; Freedman Fowler \& Horner, 2015). These definitions were maximum-clade and used 409 Brachylophosaurus, Maiasaura, and Acristavus (Gates et al., 2011) or Brachylophosaurus, 410 Probrachylophosaurus, Maiasaura, and Acristavus (Freedman Fowler \& Horner, 2015) as the 411 internal specifiers and Gryposaurus and Saurolophus as the external specifiers. The composition 412 of Brachylophosaurini and the relationships of the clade to other hadrosaurids have been stable 413 across studies since the introduction of the name. Therefore, using more than one internal 414 specifier is unnecessary. We use a definition that ensures Brachylophosaurini does not cover 415 taxa 'traditionally' comprised within Edmontosaurini, Kritosaurini, and Saurolophini.

416

417 Camptosauridae Marsh, 1885 [converted clade name]

418 Registration number: 595

419 Definition. The largest clade containing Camptosaurus dispar (Marsh, 1879) but not Iguanodon 420 bernissartensis Boulenger in Beneden, 1881. This is a maximum-clade definition. Abbreviated 421 definition: max $\nabla$ (Camptosaurus dispar [Marsh, 1879] Iguanodon bernissartensis Boulenger 422 in Beneden, 1881).

423 Reference phylogeny. Figure 13 of Madzia et al. (2020) is treated here as the primary reference 424 phylogeny. Additional reference phylogenies include Figure 20 of Verdú et al. (2018), Figure 11 425 of Santos-Cubedo et al. (2021), and Figure 9 of Verdú et al. (2020).

426 Composition. Under the primary reference phylogeny, Camptosauridae comprises

427 Camptosaurus dispar and Cumnoria prestwichii. Under alternative hypotheses, however, 428 Camptosauridae includes only a single unequivocal member, Camptosaurus dispar (e.g., Madzia 429 et al., 2020: Fig. 12).

430 Synonyms. No other taxon names are currently in use for the same or approximate clade.

431 Comments. The name Camptosauridae was first (informally) defined by Sereno (1998: 62) who 432 used the maximum-clade definition and selected Camptosaurus as the internal specifier and 
433 Parasaurolophus as the external specifier. We prefer to use Iguanodon bernissartensis as the

434 external specifier to maintain the 'node-branch triplet' ('node-stem triplet' of Sereno [1998: 52-

435 54]) comprising Ankylopollexia, Camptosauridae, and Styracosterna (all formally defined in the

436 present paper). The inclusion of a different external specifier does not change the extent of

437 Camptosauridae under any of the published phylogeny inferences.

438

439

Centrosaurinae Lambe, 1915 [converted clade name]

440 Registration number: 596

441 Definition. The largest clade containing Centrosaurus apertus Lambe, 1905 but not

442 Chasmosaurus belli (Lambe, 1902) and Triceratops horridus Marsh, 1889. This is a maximum-

443 clade definition. Abbreviated definition: $\max \nabla$ (Centrosaurus apertus Lambe, 1905

444 Chasmosaurus belli [Lambe, 1902] \& Triceratops horridus Marsh, 1889).

445 Reference phylogeny. Figure 9 of Chiba et al. (2018) is treated here as the primary reference

446 phylogeny. Additional reference phylogenies include Figure 10 of Ryan et al. (2017), Figure 13

447 of Dalman et al. (2018), Figure 10 of Wilson et al. (2020), Figure 4 of Yu et al. (2020), and

448 Figure 23 of Dalman et al. (2021).

449 Composition. Under the primary reference phylogeny, Centrosaurinae comprises

450 Albertaceratops nesmoi, Diabloceratops eatoni, Machairoceratops cronusi, Medusaceratops

451 lokii, Sinoceratops zhuchengensis, Wendiceratops pinhornensis, Xenoceratops foremostensis,

452 and members of the clades Eucentrosaura and Nasutoceratopsini.

453 Synonyms. No other taxon names are currently in use for the same or approximate clade.

454 Although Ceratops montanus may fall within the largest clade containing Centrosaurus apertus

455 but not Chasmosaurus belli and Triceratops horridus as well, the name Ceratopsinae Abel, 1919

456 has not been associated with the same contents as Centrosaurinae in the past. Therefore,

457 Ceratopsinae is not considered to be an approximate synonym of Centrosaurinae. In any case,

458 C. montanus does not seem to be diagnostic beyond Ceratopsidae at present (Dodson et al.,

459 2004; Mallon et al., 2016). Therefore, its position within the clade is uncertain. Lucas et al.

460 (2016: 202) have argued that Pachyrhinosaurinae Huene, 1950 has priority over Centrosaurinae

461 under the Article 61 of the ICZN (ICZN, 1999). However, the name Pachyrhinosaurinae has not

462 been used in the literature recently and even Lucas et al. (2016) used Centrosaurinae for the

463 clade in question.

Peer] reviewing PDF | (2021:04:60693:1:1:NEW 8 Sep 2021) 
464 Comments. The name Centrosaurinae has been (informally) defined before (Sereno, 1998;

465 Dodson et al., 2004; Sereno, 2005). These definitions were maximum-clade and used

466 Pachyrhinosaurus (Sereno, 1998), Centrosaurus (Dodson et al., 2004), or Centrosaurus apertus

467 (Sereno, 2005) as the internal specifier and Triceratops (Sereno, 1998; Dodson et al., 2004) or

468 Triceratops horridus (Sereno, 2005) as the external specifier. We apply the name

469 Centrosaurinae for the same known contents; adopting the mandatory Centrosaurus apertus as

470 the internal specifier and Chasmosaurus belli and Triceratops horridus as the external specifiers.

471

472 Centrosaurini Ryan et al., 2017 [converted clade name]

473 Registration number: 687

474 Definition. The largest clade containing Centrosaurus apertus Lambe, 1905 but not

475 Pachyrhinosaurus canadensis Sternberg, 1950. This is a maximum-clade definition. Abbreviated

476 definition: $\max \nabla$ (Centrosaurus apertus Lambe, $1905 \sim$ Pachyrhinosaurus canadensis

477 Sternberg, 1950).

478 Reference phylogeny. Figure 9 of Chiba et al. (2018) is treated here as the primary reference

479 phylogeny. Additional reference phylogenies include Figure 7 of Tykoski \& Fiorillo (2012),

480 Figure 10 of Ryan et al. (2017), Figure 13 of Dalman et al. (2018), and Figure 23 of Dalman et

481 al. (2021).

482 Composition. Under the primary reference phylogeny, Centrosaurini comprises Centrosaurus

483 apertus, Coronosaurus brinkmani, Rubeosaurus ovatus (?= Styracosaurus albertensis; see

484 Holmes et al., 2020), Spinops sternbergorum, and Styracosaurus albertensis. Under an

485 alternative hypothesis, Centrosaurini includes only a single unequivocal member, Centrosaurus

486 apertus (Wilson et al., 2020: Fig. 10). However, a Bayesian analysis of the same matrix and

487 published in the same study reconstructed Centrosaurini to comprise Centrosaurus apertus,

488 Coronosaurus brinkmani, and Spinops sternbergorum (Wilson et al., 2020: Fig. 9).

489 Synonyms. No other taxon names are currently in use for the same or approximate clade.

490 Comments. The name was first (informally) defined by Ryan et al. (2017) who applied the

491 maximum-clade definition and used Centrosaurus apertus as the internal specifier and

492 Pachyrhinosaurus canadensis as the external specifier. We formalize this definition.

493

494 Cerapoda Sereno, 1986 [converted clade name]

Peer] reviewing PDF | (2021:04:60693:1:1:NEW 8 Sep 2021) 
495

496

497

498

499

500

501

502

503

504

505

506

507

508

509

510

511

512

513

514

515

516

517

518

519

520

\section{1}

522

523

524

\section{Registration number: 597}

Definition. The smallest clade containing Iguanodon bernissartensis Boulenger in Beneden, 1881, Pachycephalosaurus wyomingensis (Gilmore, 1931), and Triceratops horridus Marsh, 1889. This is a minimum-clade definition. Abbreviated definition: $\min \nabla$ (Iguanodon bernissartensis Boulenger in Beneden, 1881 \& Pachycephalosaurus wyomingensis [Gilmore, 1931] \& Triceratops horridus Marsh, 1889).

Reference phylogeny. Figure 4 of Madzia et al. (2018) is treated here as the primary reference phylogeny. Additional reference phylogenies include Figure 16 of Han et al. (2018), Figure 25 of Herne et al. (2019), Figure 1 of Dieudonné et al. (2020), and Figure 57 of Barta \& Norell (2021).

Composition. Under the primary reference phylogeny, Cerapoda comprises members of the clades Ornithopoda and Marginocephalia.

Synonyms. No other taxon names are currently in use for the same or approximate clade.

Comments. The name Cerapoda has been (informally) defined before (Weishampel, 2004;

Butler et al., 2008). Both types of definitions, minimum-clade as well as maximum-clade, have been proposed for the name. Weishampel (2004) preferred a maximum-clade definition and used

Triceratops as the internal specifier and Ankylosaurus as the external specifier, while Butler et al. (2008) applied a minimum-clade definition, using Triceratops horridus and Parasaurolophus walkeri as the internal specifiers. Subsequent authors followed the latter definition (Boyd, 2015; Madzia et al., 2018; Herne et al., 2019; Yang et al., 2020). We apply a minimum-clade definition as well and use Iguanodon bernissartensis, Pachycephalosaurus wyomingensis, and Triceratops horridus as the internal specifiers. Note that the internal specifiers Pachycephalosaurus wyomingensis and Triceratops horridus are not included in the primary reference phylogeny. The former belongs to Pachycephalosauria (see, e.g., Dieudonné et al., 2020), while the latter is part of Ceratopsia (e.g., Morschhauser et al., 2019), both within Marginocephalia that is indicated on Figure 4 of Madzia et al. (2018).

\section{Ceratopsia Marsh, 1890 [converted clade name]}

\section{Registration number: 598}

Definition. The largest clade containing Ceratops montanus Marsh, 1888 and Triceratops horridus Marsh, 1889 but not Pachycephalosaurus wyomingensis (Gilmore, 1931). This is a 
525 maximum-clade definition. Abbreviated definition: max $\nabla$ (Ceratops montanus Marsh, 1888 \&

526 Triceratops horridus Marsh, 1889 Pachycephalosaurus wyomingensis [Gilmore, 1931]).

527 Reference phylogeny. Figure 10 of Morschhauser et al. (2019) is treated here as the primary

528 reference phylogeny. Additional reference phylogenies include Figure 16 of Han et al. (2018),

529 Figure S1 of Knapp et al. (2018), Figure 1 of Dieudonné et al. (2020), Figure 3 of Yu et al.

530 (2020), and Figure 4 of Yu et al. (2020).

531 Composition. Under the primary reference phylogeny, Ceratopsia comprises Psittacosaurus

532 spp. and members of the clades Chaoyangsauridae and Neoceratopsia.

533 Synonyms. No other taxon names are currently in use for the same or approximate clade.

534 Comments. The name Ceratopsia has been (informally) defined before (Dodson, 1997; Sereno,

535 1998; Sereno, 2005). These definitions were maximum-clade and used Ceratopsidae (Dodson,

536 1997), Triceratops (Sereno, 1998), or Triceratops horridus (Sereno, 2005) as the internal

537 specifiers and Pachycephalosauridae (Dodson, 1997), Pachycephalosaurus (Sereno, 1998), or

538 Pachycephalosaurus wyomingensis, Heterodontosaurus tucki, Hypsilophodon foxii, and

539 Ankylosaurus magniventris (Sereno, 2005) as the external specifiers. Even though the position of

540 Hypsilophodon foxii and Heterodontosaurus tucki is indeed somewhat unstable across studies

541 (see, e.g., Han et al., 2018; Madzia et al., 2018; Herne et al., 2019; Dieudonné et al., 2020; Yang

542 et al. 2020), inclusion of these taxa among the external specifiers is not necessary. We use a

543 definition similar to that of Sereno (1998) but include the mandatory Ceratops montanus as a

544 second internal specifier. Note that the internal specifier Ceratops montanus and the external

545 specifier Pachycephalosaurus wyomingensis are not included in the primary reference

546 phylogeny. The former belongs to Ceratopsidae (e.g., Mallon et al., 2016), while the latter is part

547 of Pachycephalosauria (see, e.g., Dieudonné et al., 2020).

548

549 Ceratopsidae Marsh, 1888 [converted clade name]

550 Registration number: 599

551 Definition. The smallest clade containing Centrosaurus apertus Lambe, 1905, Ceratops

552 montanus Marsh, 1888, Chasmosaurus belli (Lambe, 1902), and Triceratops horridus Marsh,

553 1889. This is a minimum-clade definition. Abbreviated definition: min $\nabla$ (Centrosaurus apertus

554 Lambe, 1905 \& Ceratops montanus Marsh, 1888 \& Chasmosaurus belli [Lambe, 1902] \&

555 Triceratops horridus Marsh, 1889).

Peer] reviewing PDF | (2021:04:60693:1:1:NEW 8 Sep 2021) 
556 Reference phylogeny. Figure 4 of Yu et al. (2020) is treated here as the primary reference

557 phylogeny. Additional reference phylogenies include Figure 14 of Mallon et al. (2016), Figure

558 S1 of Knapp et al. (2018), Figure 9a of Fowler \& Freedman Fowler (2020), Figure 10 of Wilson

559 et al. (2020), and Figure 3 of Yu et al. (2020).

560 Composition. Under the primary reference phylogeny, Ceratopsidae comprises members of the 561 clades Centrosaurinae and Chasmosaurinae.

562 Synonyms. No other taxon names are currently in use for the same or approximate clade.

563 Comments. The name Ceratopsidae has been (informally) defined before (Sereno, 1998,

564 Dodson et al., 2004; Sereno, 2005). These definitions were minimum-clade and used Triceratops

565 and Pachyrhinosaurus (Sereno, 1998), Triceratops and Centrosaurus (Dodson et al., 2004), and

566 Triceratops horridus and Pachyrhinosaurus canadensis (Sereno, 2005) as the internal specifiers.

567 Considering that Ceratopsidae 'traditionally' contains two subclades, Centrosaurinae and

568 Chasmosaurinae, we include the nomenclatural types of these clades, Centrosaurus apertus and

569 Chasmosaurus belli, respectively, as the internal specifiers, and additionally add Triceratops

570 horridus, a common specifier in the nomenclature of ceratopsian clades and the only taxon that

571 has always been used as an internal specifier in the definition of Ceratopsidae. Finally, we also

572 include a fourth internal specifier, the mandatory Ceratops montanus. Even though the taxon is

573 considered a nomen dubium (e.g., Dodson et al., 2004; Mallon et al., 2016), its placement within

574 the smallest clade comprising centrosaurines and chasmosaurines does not appear to be

575 questionable (see, e.g., Mallon et al., 2016).

576

577

Ceratopsoidea Hay, 1902 [converted clade name]

578 Registration number: 601

579 Definition. The largest clade containing Ceratops montanus Marsh, 1888 and Triceratops

580 horridus Marsh, 1889 but not Protoceratops andrewsi Granger \& Gregory, 1923. This is a

581 maximum-clade definition. Abbreviated definition: $\max \nabla$ (Ceratops montanus Marsh, 1888 \&

582 Triceratops horridus Marsh, 1889 Protoceratops andrewsi Granger \& Gregory, 1923).

583 Reference phylogeny. Figure 4 of Yu et al. (2020) is treated here as the primary reference

584 phylogeny. Additional reference phylogenies include Figure S1 of Knapp et al. (2018), Figure 10

585 of Morschhauser et al. (2019), and Figure 3 of Yu et al. (2020).

Peer] reviewing PDF | (2021:04:60693:1:1:NEW 8 Sep 2021) 
586 Composition. Under the primary reference phylogeny, Ceratopsoidea comprises

587 Turanoceratops tardabilis, Zuniceratops christopheri, and members of the clade Ceratopsidae.

588 Synonyms. No other taxon names are currently in use for the same or approximate clade.

589 Comments. The name Ceratopsoidea has been (informally) defined before by Sereno (1998,

590 2005) who applied a maximum-clade definition and used Triceratops horridus as the internal

591 specifier and Protoceratops andrewsi as the external specifier. We include an additional internal

592 specifier, the mandatory Ceratops montanus.

593

594 Chaoyangsauridae Zhao et al., 1999 [converted clade name]

595 Registration number: 602

596 Definition. The largest clade containing Chaoyangsaurus youngi Zhao et al., 1999 but not

597 Psittacosaurus mongoliensis Osborn, 1923 and Triceratops horridus Marsh, 1889. This is a

598 maximum-clade definition. Abbreviated definition: $\max \nabla$ (Chaoyangsaurus youngi Zhao et al.,

5991999 Psittacosaurus mongoliensis Osborn, 1923 \& Triceratops horridus Marsh, 1889).

600 Reference phylogeny. Figure 10 of Morschhauser et al. (2019) is treated here as the primary

601 reference phylogeny. Additional reference phylogenies include Figure 10 of Han et al. (2015),

602 Figure 15 of Han et al. (2018), and Figure 3 of Yu et al. (2020).

603 Composition. Under the primary reference phylogeny, Chaoyangsauridae comprises

604 Chaoyangsaurus youngi, Hualianceratops wucaiwanensis, Xuanhuaceratops niei, and Yinlong 605 downsi.

606 Synonyms. No other taxon names are currently in use for the same or approximate clade.

607 Comments. The name Chaoyangsauridae has been (informally) defined before by Han et al.

608 (2015) who applied a maximum-clade definition and used Chaoyangsaurus youngi as the 609 internal specifier and Triceratops horridus and Psittacosaurus mongoliensis as the external 610 specifiers. We formalize this definition.

611

612 Chasmosaurinae Lambe, 1915 [converted clade name]

613 Registration number: 603

614 Definition. The largest clade containing Chasmosaurus belli (Lambe, 1902) and Triceratops

615 horridus Marsh, 1889 but not Centrosaurus apertus Lambe, 1905. This is a maximum-clade 
616 definition. Abbreviated definition: $\max \nabla$ (Chasmosaurus belli [Lambe, 1902] \& Triceratops

617 horridus Marsh, 1889 Centrosaurus apertus Lambe, 1905).

618 Reference phylogeny. Figure 9a of Fowler \& Freedman Fowler (2020) is treated here as the

619 primary reference phylogeny. Additional reference phylogenies include Figure 3 of Brown \&

620 Henderson (2015), Figure 14 of Mallon et al. (2016), Figure S1 of Knapp et al. (2018), Figure 3

621 of Campbell et al. (2019), and Figure 4 of Yu et al. (2020).

622 Composition. Under the primary reference phylogeny, Chasmosaurinae comprises

623 Agujaceratops mariscalensis, Anchiceratops ornatus, Arrhinoceratops brachyops,

624 Bravoceratops polyphemus, Chasmosaurus spp., Coahuilaceratops magnacuerna,

625 Kosmoceratops richardsoni, Navajoceratops sullivani, Pentaceratops sternbergii, Terminocavus

626 sealyi, Utahceratops gettyi, Vagaceratops irvinensis, and members of the clade Triceratopsini.

627 Synonyms. The taxon Ceratops montanus may also fall within the largest clade containing

628 Chasmosaurus belli and Triceratops horridus but not Centrosaurus apertus (see, e.g., Mallon et

629 al., 2016). In such case, Ceratopsinae Abel, 1919 would be an approximate synonym. Though

630 the name has been advocated to be the proper name for the clade (it has been [informally]

631 defined by Sereno, 1998 and Sereno, 2005), it was actually introduced four years later than

632 Chasmosaurinae. Note that the Principle of Coordination, which would make Ceratopsinae

633 attributable to Marsh (1888), rather than to Abel (1919), does not apply under the ICPN (see

634 Note 9.15A.3). Therefore, Ceratopsinae would not have priority over Chasmosaurinae under the

$635 I C P N$. Anyway, C. montanus does not seem to be diagnostic beyond Ceratopsidae at present

636 (Mallon et al., 2016), and its position within the clade is thus uncertain.

637 Comments. The name Chasmosaurinae has been (informally) defined before by Dodson et al.

638 (2004) who applied a maximum-clade definition and used Triceratops as the internal specifier

639 and Centrosaurus as the external specifier. We apply the name Chasmosaurinae for the same

640 known contents; adopting Triceratops horridus and the mandatory Chasmosaurus belli as the

641 internal specifiers and Centrosaurus apertus as the external specifier.

642

643 Clypeodonta Norman, 2014 [converted clade name]

644 Registration number: 604

645 Definition. The smallest clade within Ornithopoda containing Edmontosaurus regalis Lambe, 6461917 and Hypsilophodon foxii Huxley, 1869. This is a minimum-clade definition. Abbreviated 
647 definition: $\min \nabla \in$ Ornithopoda (Edmontosaurus regalis Lambe, 1917 \& Hypsilophodon foxii

648 Huxley, 1869).

649 Reference phylogeny. Figure 50 of Norman (2015) is treated here as the primary reference

650 phylogeny. Additional reference phylogenies include Figure 25 of Herne et al. (2019) and Figure 6512 of Dieudonné et al. (2020).

652 Composition. Under the primary reference phylogeny, Clypeodonta comprises a clade formed

653 by Hypsilophodon foxii, Rhabdodontidae, and Tenontosaurus spp., and a clade uniting

654 Dryosauridae and Ankylopollexia (termed Iguanodontia in Norman, 2015). However, see

655 'Comments' below for discussion of potential alternative composition of Clypeodonta.

656 Synonyms. No other taxon names are currently in use for the same or approximate clade.

657 Iguanodontia, as reconstructed, for example, by Madzia et al. (2020) covers a similar taxic

658 composition; though the topology of Madzia et al. (2020) differs from that of the primary

659 reference phylogeny of Clypeodonta significantly.

660 Comments. The name Clypeodonta was claimed as being new in two different studies (Norman, 661 2014: 29; Norman, 2015: 102), although Norman (2015: 170) also cites Norman (2014) as the

662 establishing reference. The use of the name Clypeodonta differed across studies. Originally, 663 Norman $(2014,2015)$ intended to use it for a subclade of Ornithopoda that (approximately) 664 comprises Hypsilophodon foxii and its relatives, and ornithopods later-diverging than H. foxii, 665 and (informally) defined the name as pertaining to either, the branch of "Parasaurolophus 666 walkeri and all taxa more closely related to P. walkeri than to Thescelosaurus neglectus" 667 (Norman, 2014: 29) and the node of "Hypsilophodon foxii, Edmontosaurus regalis, their most 668 recent common ancestor, and all of its descendants" (Norman, 2015: 170). In both these studies, 669 Clypeodonta is said (Norman, 2014: 29) or figured (Norman, 2015: Fig. 50) to cover the same 670 known contents although neither of the studies included taxa in their analyses that would fall 671 outside the clade (except for Lesothosaurus diagnosticus). Madzia et al. (2018) followed the 672 definition of Norman (2015). In their phylogenetic analysis, however, the name covers a much 673 broader contents as one of the internal specifiers of Clypeodonta, Hypsilophodon foxii, is 674 reconstructed outside Cerapoda in that study (Madzia et al. 2018: Fig. 4). Still, Madzia et al. 675 (2018: Appendix 1) stated that as Clypeodonta was a relatively new name with no 'traditional' 676 meaning, they saw no reason for its redefinition. They also noted, though, that "given the 
677 unstable position of $H$. foxii among neornithischians, the name might have only limited utility" 678 (Madzia et al., 2018: Appendix 1).

679 Here we define the name Clypeodonta using the minimum-clade definition of Norman (2015).

680 However, by including the part "within Ornithopoda" in the definition, we restrict the use of

681 Clypeodonta only when $H$. foxii represents an ornithopod (see Article 11.14 of the ICPN),

682 following the original intent of Norman $(2014,2015)$.

683

684 Coronosauria Sereno, 1986 [converted clade name]

685 Registration number: 605

686 Definition. The smallest clade containing Protoceratops andrewsi Granger \& Gregory, 1923 and 687 Triceratops horridus Marsh, 1889. This is a minimum-clade definition. Abbreviated definition: $688 \min \nabla$ (Protoceratops andrewsi Granger \& Gregory, 1923 \& Triceratops horridus Marsh, 1889).

689 Reference phylogeny. Figure 10 of Morschhauser et al. (2019) is treated here as the primary

690 reference phylogeny. Additional reference phylogenies include Figure S1 of Knapp et al. (2018),

691 Figure 8A of Arbour et al. (2019), Figure 3 of Yu et al. (2020), and Figure 4 of Yu et al. (2020).

692 Composition. Under the primary reference phylogeny, Coronosauria comprises members of the 693 clades Protoceratopsidae and Ceratopsoidea.

694 Synonyms. No other taxon names are currently in use for the same or approximate clade.

695 Comments. The name Coronosauria has been (informally) defined before by Sereno (1998, 696 2005) who applied the minimum-clade definition and used Triceratops horridus and

697 Protoceratops andrewsi as the internal specifiers. We formalize this definition.

698

699 Corythosauria [new clade name]

700 Registration number: 746

701 Definition. The smallest clade containing Corythosaurus casuarius Brown, 1914a,

702 Lambeosaurus lambei Parks, 1923, and Parasaurolophus walkeri Parks, 1922. This is a

703 minimum-clade definition. Abbreviated definition: min $\nabla$ (Corythosaurus casuarius Brown,

704 1914a \& Lambeosaurus lambei Parks, 1923 \& Parasaurolophus walkeri Parks, 1922).

705 Etymology. Derived from the stem of Corythosaurus Brown, 1914a, the name of an included 706 taxon, which combines the Greek words korythos (helmet) and sauros (lizard, reptile). 
707 Reference phylogeny. Figure 18 of Prieto-Márquez et al. (2020) is treated here as the primary

708 reference phylogeny. Additional reference phylogenies include Figure 5 of Kobayashi et al.

709 (2019), Figure 11 of Prieto-Márquez et al. (2019), Figure 9 of Zhang et al. (2019), Figure 5 of

710 Zhang et al. (2020), Figure 7 of Kobayashi et al. (2021), and Figure 10 of Longrich et al. (2021).

711 Composition. Under the primary reference phylogeny, Corythosauria comprises members of the

712 clades Lambeosaurini and Parasaurolophini.

713 Synonyms. No other taxon names are currently in use for the same or approximate clade.

714 Comments. The name Corythosauria is established for the well-supported node uniting

715 Lambeosaurini and Parasaurolophini, two lambeosaurine clades characterized by their

716 distinctive, 'crested' crania.

717

Dryomorpha Sereno, 1986 [converted clade name]

719 Registration number: 606

720 Definition. The smallest clade containing Dryosaurus altus (Marsh, 1878) and Iguanodon

721 bernissartensis Boulenger in Beneden, 1881. This is a minimum-clade definition. Abbreviated

722 definition: min $\nabla$ (Dryosaurus altus [Marsh, 1878] \& Iguanodon bernissartensis Boulenger in

723 Beneden, 1881).

724 Reference phylogeny. Figure 12 of Madzia et al. (2020) is treated here as the primary reference

725 phylogeny. Additional reference phylogenies include Figure 20 of Verdú et al. (2018), Figure 2

726 of Dieudonné et al. (2020), Figure 11 of Santos-Cubedo et al. (2021), and Figure 9 of Verdú et

727 al. (2020).

728 Composition. Under the primary reference phylogeny, Dryomorpha comprises members of the

729 clades Dryosauridae and Ankylopollexia.

730 Synonyms. No other taxon names are currently in use for the same or approximate clade.

731 Comments. The name Dryomorpha was first (informally) defined by Sereno (2005) who

732 attributed the name to "[t]he most inclusive clade containing Dryosaurus altus (Marsh 1878) and

733 Parasaurolophus walkeri Parks 1922". However, due to the use of 'most', rather than 'least',

734 such definition makes the name inapplicable within Ornithischia. Boyd (2015) later corrected the

735 wording and proposed a minimum-clade definition using the same taxa as the internal specifiers.

736 Here we use the same type of definition but replace P. walkeri with I. bernissartensis. This taxon

737 has always been considered a part of Dryomorpha. 
739 Dryosauridae Milner \& Norman, 1984 [converted clade name]

740 Registration number: 607

741 Definition. The largest clade containing Dryosaurus altus (Marsh, 1878) but not Iguanodon

742 bernissartensis Boulenger in Beneden, 1881. This is a maximum-clade definition. Abbreviated

743 definition: $\max \nabla$ (Dryosaurus altus [Marsh, 1878] Iguanodon bernissartensis Boulenger in

744 Beneden, 1881).

745 Reference phylogeny. Figure 12 of Madzia et al. (2020) is treated here as the primary reference

746 phylogeny. Additional reference phylogenies include Figure 20 of Verdú et al. (2018), Figure 57

747 of Barta \& Norell (2021), Figure 11 of Santos-Cubedo et al. (2021), and Figure 9 of Verdú et al. 748 (2020).

749 Composition. Under the primary reference phylogeny, Dryosauridae comprises Callovosaurus

750 leedsi, 'Camptosaurus' valdensis, Dryosaurus altus, Dysalotosaurus lettowvorbecki,

751 Elrhazosaurus nigeriensis, Eousdryosaurus nanohallucis, and Valdosaurus canaliculatus.

752 Synonyms. No other taxon names are currently in use for the same or approximate clade.

753 Comments. Dryosauridae was first (informally) defined by Sereno (1998: 61) who used the

754 maximum-clade definition and Dryosaurus altus as the internal specifier and Parasaurolophus

755 walkeri as the external specifier. Here we use the same type of definition but replace $P$. walkeri

756 with I. bernissartensis. This taxon has always been considered outside Dryosauridae.

757

758 Edmontosaurini Glut, 1997 [converted clade name]

759 Registration number: 608

760 Definition. The largest clade containing Edmontosaurus regalis Lambe, 1917 but not

761 Brachylophosaurus canadensis Sternberg, 1953, Hadrosaurus foulkii Leidy, 1858, Kritosaurus

762 navajovius Brown, 1910, and Saurolophus osborni Brown, 1912. This is a maximum-clade

763 definition. Abbreviated definition: $\max \nabla$ (Edmontosaurus regalis Lambe, 1917

764 Brachylophosaurus canadensis Sternberg, 1953 \& Hadrosaurus foulkii Leidy, 1858 \&

765 Kritosaurus navajovius Brown, 1910 \& Saurolophus osborni Brown, 1912).

766 Reference phylogeny. Figure 18 of Prieto-Márquez et al. (2020) is treated here as the primary

767 reference phylogeny. Additional reference phylogenies include Figure 5 of Kobayashi et al. 
768 (2019), Figure 11 of Prieto-Márquez et al. (2019), Figure 9 of Zhang et al. (2019), Figure 5 of

769 Zhang et al. (2020), Figure 7 of Kobayashi et al. (2021), and Figure 10 of Longrich et al. (2021).

770 Composition. Under the primary reference phylogeny, Edmontosaurini comprises

771 Edmontosaurus spp., Kerberosaurus manakini, Kundurosaurus nagornyi, and Shantungosaurus 772 giganteus.

773 Synonyms. No other taxon names are currently in use for the same or approximate clade.

774 Comments. The name Edmontosaurini has been (informally) defined before (Sereno, 2005;

775 Xing et al., 2014). Sereno (2005) applied the maximum-clade definition and used

776 Edmontosaurus regalis as the internal specifier and Maiasaura peeblesorum and Saurolophus

777 osborni as the external specifiers. In turn, Xing et al. (2014) applied a minimum-clade definition,

778 with Edmontosaurus and Kerberosaurus as the internal specifiers. We formalize a maximum-

779 clade definition similar to that of Sereno (2005) but replace M. peeblesorum with

780 Brachylophosaurus canadensis, as the representative of Brachylophosaurini, and further add

781 Kritosaurus navajovius and Hadrosaurus foulkii.

782

783

784

Elasmaria Calvo et al., 2007 [converted clade name]

Registration number: 609

785

Definition. The smallest clade containing Macrogryphosaurus gondwanicus Calvo et al., 2007

786

and Talenkauen santacrucensis Novas et al., 2004, provided that it does not include

787

Hypsilophodon foxii Huxley, 1869, Iguanodon bernissartensis Boulenger in Beneden, 1881, or

Thescelosaurus neglectus Gilmore, 1913. This is a minimum-clade definition. Abbreviated

789

definition: $\min \nabla$ (Macrogryphosaurus gondwanicus Calvo et al., 2007 \& Talenkauen

790

santacrucensis Novas et al., $2004 \mid \sim$ Hypsilophodon foxii Huxley, 1869 v Iguanodon

791

bernissartensis Boulenger in Beneden, $1881 \vee$ Thescelosaurus neglectus Gilmore, 1913).

792

Reference phylogeny. Figure 31 of Rozadilla et al. (2019) is treated here as the primary

reference phylogeny. Additional reference phylogenies include Figure 4 of Madzia et al. (2018),

794 Figure 26 of Herne et al. (2019), Figure 2 of Dieudonné et al. (2020), and Figure 57 of Barta \&

795 Norell (2021).

796 Composition. Under the primary reference phylogeny, Elasmaria comprises Anabisetia

797 saldiviai, Atlascopcosaurus loadsi, Fulgurotherium austral, Gasparinisaura cincosaltensis, 
798 Kangnasaurus coetzeei, Macrogryphosaurus gondwanicus, Morrosaurus antarcticus,

799 Notohypsilophodon comodorensis, Quantassaurus intrepidus, and Trinisaura santamartaensis.

800 Synonyms. No other taxon names are currently in use for the same or approximate clade.

801 Comments. The name Elasmaria has been (informally) defined before (Calvo et al., 2007;

802 Herne et al., 2019). The definition proposed by Calvo et al. (2007) was minimum-clade, while

803 the definition of Herne et al. (2019) was maximum-clade. However, both studies used

804 Talenkauen santacrucensis and Macrogryphosaurus gondwanicus as the internal specifiers.

805 Herne et al. (2019) proposed to add Iguanodon bernissartensis and Hypsilophodon foxii as the

806 external specifiers to maintain the use of the name Elasmaria to the 'traditional' contents under a

807 hypothesis in which one of the internal specifiers was reconstructed, for example, closer to

808 iguanodontians. We keep the use of a minimum-clade definition (as first proposed for the name).

809 However, even though all phylogenetic analyses consistently reconstruct close relationships

810 between T. santacrucensis and M. gondwanicus, we follow Herne et al. (2019) in that the

811 unsettled placement of elasmarians on the neornithischian phylogenetic tree warrants addition of

812 external specifiers. We include Iguanodon bernissartensis and Hypsilophodon foxii as the

813 external specifiers (following Herne et al., 2019) and further add a third external specifier,

814 Thescelosaurus neglectus, to reflect that elasmarians were already inferred as a clade within

815 Thescelosaurinae, as the sister taxon to Thescelosaurus spp. (Boyd, 2015).

816

817 Eucentrosaura Chiba et al., 2018 [converted clade name]

818 Registration number: 688

819 Definition. The smallest clade containing Centrosaurus apertus Lambe, 1905 and

820 Pachyrhinosaurus canadensis Sternberg, 1950. This is a minimum-clade definition. Abbreviated

821 definition: $\min \nabla$ (Centrosaurus apertus Lambe, 1905 \& Pachyrhinosaurus canadensis

822 Sternberg, 1950).

823 Reference phylogeny. Figure 9 of Chiba et al. (2018) is treated here as the primary reference

824 phylogeny. Additional reference phylogenies include Figure 7 of Tykoski \& Fiorillo (2012),

825 Figure 10 of Ryan et al. (2017), Figure 13 of Dalman et al. (2018), and Figure 23 of Dalman et

826 al. (2021).

827 Composition. Under the primary reference phylogeny, Eucentrosaura comprises members of the 828 clades Centrosaurini and Pachyrhinosaurini. 
829 Synonyms. No other taxon names are currently in use for the same or approximate clade.

830 Comments. The name was first (informally) defined by Chiba et al. (2018) who applied the

831 minimum-clade definition and used Centrosaurus apertus and Pachyrhinosaurus canadensis as

832 the internal specifiers. We formalize this definition.

833

834 Euceratopsia [new clade name]

835 Registration number: 610

836 Definition. The smallest clade containing Leptoceratops gracilis Brown, 1914b, Protoceratops

837 andrewsi Granger \& Gregory, 1923, and Triceratops horridus Marsh, 1889. This is a minimum-

838 clade definition. Abbreviated definition: $\min \nabla$ (Leptoceratops gracilis Brown, 1914b \&

839 Protoceratops andrewsi Granger \& Gregory, 1923 \& Triceratops horridus Marsh, 1889).

840 Etymology. Derived from the Greek eu- (true) and formed to show its association to members of

841 Ceratopsia. Note that Euceratopsia does not derive from the name Ceratops Marsh, 1888, and,

842 as such, the taxon does not have to be the internal specifier in the used definition.

843 Reference phylogeny. Figure 4 of Yu et al. (2020) is treated here as the primary reference

844 phylogeny. Additional reference phylogenies include Figure 16 of Han et al. (2018), Figure S1 of

845 Knapp et al. (2018), Figure 10 of Morschhauser et al. (2019), and Figure 3 of Yu et al. (2020).

846 Composition. Under the primary reference phylogeny, Euceratopsia comprises members of the

847 clades Leptoceratopsidae and Coronosauria.

848 Synonyms. The name Coronosauria Sereno, 1986 covers the same contents under the topology

849 of You \& Dodson (2004). However, see 'Comments'. No other taxon names are currently in use

850 for the same or approximate clade.

851 Comments. The name Euceratopsia is established for the well-supported node uniting the three

852 latest-diverging clades of ceratopsians - Leptoceratopsidae, Protoceratopsidae, and

853 Ceratopsoidea. The monophyly of the grouping is supported by all recently published

854 phylogenies that infer Euceratopsia to branch into two clades - leptoceratopsids and coronosaurs

855 (protoceratopsids + ceratopsoids). Both these clades comprise representatives that are very close

856 or survived to the Cretaceous/Paleogene mass extinction event (Fowler, 2017: S1 Table). It is

857 worth noting that You \& Dodson (2004) reconstructed leptoceratopsids to be the sister taxon to

858 Ceratopsoidea, and Protoceratopsidae to be the sister taxon to Leptoceratopsidae +

Peer) reviewing PDF | (2021:04:60693:1:1:NEW 8 Sep 2021) 
859 Ceratopsoidea. Under such topology, Euceratopsia becomes a heterodefinitional synonym of

860 Coronosauria, with the latter having priority.

861

862 Euhadrosauria Weishampel et al., 1993 [converted clade name]

863 Registration number: 611

864 Definition. The smallest clade containing Lambeosaurus lambei Parks, 1923 and Saurolophus

865 osborni Brown, 1912, provided that it does not include Hadrosaurus foulkii Leidy, 1858. This is

866 a minimum-clade definition. Abbreviated definition: $\min \nabla$ (Lambeosaurus lambei Parks, 1923

867 \& Saurolophus osborni Brown, 1912| Hadrosaurus foulkii Leidy, 1858).

868 Reference phylogeny. Figure 18 of Prieto-Márquez et al. (2020) is treated here as the primary

869 reference phylogeny. Additional reference phylogenies include Figure 11 of Prieto-Márquez et

870 al. (2019), Figure 9 of Zhang et al. (2019), Figure 7 of Kobayashi et al. (2021), Figure 10 of

871 Longrich et al. (2021), and Figure 11 of McDonald et al. (2021).

872 Composition. Under the primary reference phylogeny, Euhadrosauria comprises members of 873 the clades Saurolophinae and Lambeosaurinae.

874 Synonyms. The name Hadrosauridae Cope, 1869 is an approximate synonym of Euhadrosauria.

875 If Hadrosaurus foulkii nests within the smallest clade containing Saurolophus osborni and

876 Lambeosaurus lambei, and within the 'Saurolophus branch' of the clade (see the entry for the

877 name Saurolophinae), the name Hadrosauridae is used for the node instead, and Euhadrosauria

878 becomes inapplicable. Additionally, the name Saurolophidae has been used for the same

879 contents as well (see 'Comments').

880 Comments. The history and application of Euhadrosauria is complicated and has been

881 thoroughly described and discussed by Madzia et al. (2020:14-16). We therefore refer to that

882 study for details.

883

884 Euiguanodontia Coria \& Salgado, 1996 [converted clade name]

885 Registration number: 612

886 Definition. The smallest clade containing Camptosaurus dispar (Marsh, 1879), Dryosaurus altus

887 (Marsh, 1878), and Gasparinisaura cincosaltensis Coria \& Salgado, 1996, provided that it does 888 not include Tenontosaurus tilletti Ostrom, 1970. This is a minimum-clade definition.

889 Abbreviated definition: min $\nabla$ (Camptosaurus dispar [Marsh, 1879] \& Dryosaurus altus [Marsh, 
890 1878] \& Gasparinisaura cincosaltensis Coria and Salgado, 1996 | Tenontosaurus tilletti

891 Ostrom, 1970).

892 Reference phylogeny. Figure 13 of Coria \& Salgado (1996) is treated here as the primary

893 reference phylogeny.

894 Composition. Under the primary reference phylogeny, Euiguanodontia comprises

895 Gasparinisaura and members of the clades Dryosauridae and Ankylopollexia.

896 Synonyms. No other taxon names are currently in use for the same or approximate clade.

897 Comments. The name Euiguanodontia is applicable only on the condition that G. cincosaltensis,

898 D. altus, and C. dispar form a clade exclusive of T. tilletti, as originally used by Coria \& Salgado

899 (1996). We follow the definition advocated by Madzia et al. (2018: Appendix 1) and refer to that

900 study for additional comments. Note also that Euiguanodontia must be a subclade of

901 Iguanodontia under the proposed definition because T. tilletti is an internal specifier in the

902 definition of the name. Note that the internal specifiers Dryosaurus altus and Camptosaurus

903 dispar are not included in the primary reference phylogeny. The former belongs to Dryosauridae

904 (e.g., Madzia et al., 2018), while the latter is part of Ankylopollexia (see, e.g., Madzia et al.,

905 2020). Both these clades are indicated on Figure 13 of Coria \& Salgado (1996).

906

907 Euornithopoda Sereno, 1986 [converted clade name]

908 Registration number: 613

909 Definition. The largest clade within Ornithopoda containing Iguanodon bernissartensis

910 Boulenger in Beneden, 1881 but not Heterodontosaurus tucki Crompton and Charig, 1962. This

911 is a maximum-clade definition. Abbreviated definition: $\max \nabla \in$ Ornithopoda (Iguanodon

912 bernissartensis Boulenger in Beneden, 1881 Heterodontosaurus tucki Crompton and Charig,

913 1962).

914 Reference phylogeny. Figure 1 of Sereno (1999) is treated here as the primary reference

915 phylogeny.

916 Composition. Under the primary reference phylogeny, Euornithopoda comprises Tenontosaurus

917 spp. and members of the clades Ankylopollexia, Dryosauridae, and Hypsilophodontidae.

918 Synonyms. No other taxon names are currently in use for the same or approximate clade.

919 Comments. The name Euornithopoda has been (informally) defined before (Sereno, 1998;

920 Sereno, 2005). These definitions were maximum-clade and used Parasaurolophus as the internal 
921 specifier and Heterodontosaurus tucki, Pachycephalosaurus wyomingensis, Triceratops

922 horridus, and Ankylosaurus magniventris (Sereno, 2005) as the external specifiers. Here we 923 define the name Euornithopoda using a similar maximum-clade definition as that of Sereno

924 (1998) but replace Parasaurolophus with Iguanodon bernissartensis. Also, by including the part

925 "within Ornithopoda" in the definition, we restrict the use of Euornithopoda to the branch only

926 when Heterodontosaurus tucki represents an ornithopod (see Article 11.14 of the ICPN), thus

927 maintaining the 'traditional' use (Sereno, 1998; Sereno, 2005).

928

929 Eurypoda Sereno, 1986 [converted clade name]

930 Registration number: 614

931 Definition. The smallest clade containing Ankylosaurus magniventris Brown, 1908 and

932 Stegosaurus stenops Marsh, 1887. This is a minimum-clade definition. Abbreviated definition:

$933 \min \nabla$ (Ankylosaurus magniventris Brown, 1908 \& Stegosaurus stenops Marsh, 1887).

934 Reference phylogeny. Figure 3 of Thompson et al. (2012) is treated here as the primary

935 reference phylogeny. Additional reference phylogenies include Figure 16 of Han et al. (2018)

936 and Figure 1 of Dieudonné et al. (2020).

937 Composition. Under the primary reference phylogeny, Eurypoda comprises members of the

938 clades Ankylosauria and Stegosauria.

939 Synonyms. No other taxon names are currently in use for the same or approximate clade.

940 Comments. The name Eurypoda has been (informally) defined before by Sereno (1998) who

941 used Ankylosaurus and Stegosaurus as the internal specifiers. Since Eurypoda has never been

942 proposed an alternative use, we formalize this definition. Note that the internal specifier

943 Stegosaurus stenops is not included in the primary reference phylogeny. The taxon is most

944 closely related to the clade comprising the operational taxonomic units (OTUs) Stegosaurus

945 armatus (nomen dubium according to Galton, 2010; S. armatus has long been the type species of

946 Stegosaurus but was replaced by S. stenops as the type through an ICZN ruling [ICZN, 2013])

947 and Huayangosaurus taibaii (see, e.g., Maidment et al., 2020).

948

949 Genasauria Sereno, 1986 [converted clade name]

950 Registration number: 615

Peer] reviewing PDF | (2021:04:60693:1:1:NEW 8 Sep 2021) 
951 Definition. The smallest clade containing Ankylosaurus magniventris Brown, 1908, Iguanodon

952 bernissartensis Boulenger in Beneden, 1881, Stegosaurus stenops Marsh, 1887, and Triceratops

953 horridus Marsh, 1889. This is a minimum-clade definition. Abbreviated definition: $\min \nabla$

954 (Ankylosaurus magniventris Brown, 1908 \& Iguanodon bernissartensis Boulenger in Beneden,

9551881 \& Stegosaurus stenops Marsh, 1887 \& Triceratops horridus Marsh, 1889).

956 Reference phylogeny. Figure 16 of Han et al. (2018) is treated here as the primary reference

957 phylogeny. Additional reference phylogenies include Figure 4 of Madzia et al. (2018), Figure 25

958 of Herne et al. (2019), Figure 1 of Dieudonné et al. (2020), Figure 12 of Yang et al. (2020), and

959 Figure 57 of Barta \& Norell (2021).

960 Composition. Under the primary reference phylogeny, Genasauria comprises members of the

961 clades Neornithischia and Thyreophora.

962 Synonyms. No other taxon names are currently in use for the same or approximate clade.

963 Comments. The name Genasauria has been (informally) defined before (Currie and Padian,

964 1997; Sereno, 1998; Sereno, 2005; Butler et al., 2008). These definitions were minimum-clade

965 and used Thyreophora and Cerapoda (Currie and Padian, 1997), Ankylosaurus and Triceratops

966 (Sereno, 1998), Ankylosaurus magniventris, Triceratops horridus, and Parasaurolophus walkeri

967 (Sereno, 2005), and Ankylosaurus magniventris, Stegosaurus stenops, Triceratops horridus,

968 Parasaurolophus walkeri, and Pachycephalosaurus wyomingensis (Butler et al., 2008) as the

969 internal specifiers. In order to maintain the 'traditional' concept of Genasauria as a clade

970 comprising Neornithischia and Thyreophora, the internal specifiers in the definition of

971 Genasauria are used from among the taxa representing the four major subclades - Ornithopoda

972 (Iguanodon bernissartensis), Marginocephalia (Triceratops horridus), Ankylosauria

973 (Ankylosaurus magniventris), and Stegosauria (Stegosaurus stenops). Addition of P.

974 wyomingensis as another internal specifier (to include representatives of both marginocephalian

975 clades - Ceratopsia and Pachycephalosauria) is considered unnecessary because

976 pachycephalosaurs have always been inferred to be part of Genasauria as defined herein. Note

977 that the internal specifiers Ankylosaurus magniventris and Triceratops horridus are not included

978 in the primary reference phylogeny. The former belongs to Ankylosauria within Thyreophora

979 (see, e.g., Thompson et al., 2012), while the latter is part of Ceratopsia (e.g., Morschhauser et al., 980 2019).

981

Peer] reviewing PDF | (2021:04:60693:1:1:NEW 8 Sep 2021) 
982 Hadrosauridae Cope, 1869 [converted clade name]

983 Registration number: 616

984 Definition. The smallest clade containing Hadrosaurus foulkii Leidy, 1858, Lambeosaurus

985 lambei Parks, 1923, and Saurolophus osborni Brown, 1912. This is a minimum-clade definition.

986 Abbreviated definition: min $\nabla$ (Hadrosaurus foulkii Leidy, 1858 \& Lambeosaurus lambei Parks,

9871923 \& Saurolophus osborni Brown, 1912).

988 Reference phylogeny. Figure 18 of Prieto-Márquez et al. (2020) is treated here as the primary

989 reference phylogeny. Additional reference phylogenies include Figure 5 of Kobayashi et al.

990 (2019), Figure 11 of Prieto-Márquez et al. (2019), Figure 9 of Zhang et al. (2019), Figure 5 of

991 Zhang et al. (2020), Figure 7 of Kobayashi et al. (2021), and Figure 10 of Longrich et al. (2021).

992 Composition. Under the primary reference phylogeny, Hadrosauridae comprises Hadrosaurus

993 foulkii, Eotrachodon orientalis, Latirhinus uitstlani, Aquilarhinus palimentus, and members of

994 the clades Saurolophinae and Lambeosaurinae.

995 Synonyms. Several taxon names have been historically or recently used as approximate

996 synonyms of Hadrosauridae. Of these, only the names Saurolophidae and Euhadrosauria have

997 recently been attributed to a clade of the same or a similar composition (e.g., Prieto-Márquez,

998 2010; Verdú et al., 2018; Zhang et al., 2019; Madzia et al., 2020; Prieto-Márquez et al., 2020;

999 Verdú et al., 2020; Zhang et al., 2020; Kobayashi et al., 2021; Ramírez-Velasco et al., 2021). See

1000 'Comments' below.

1001 Comments. The use of Hadrosauridae and other names applied to the same or similar clades

1002 (Saurolophidae and Euhadrosauria) have been thoroughly described and discussed by Madzia et

1003 al. (2020: 14-16) who recommended to use Hadrosauridae for the smallest clade containing $H$.

1004 foulkii, S. osborni, and L. lambei; Euhadrosauria for the smallest clade containing S. osborni and

1005 L. lambei; and to abandon Saurolophidae. Note that under some phylogenies, in which H. foulkii

1006 is reconstructed within the smallest clade containing S. osborni and L. lambei, the names

1007 Hadrosauridae and Euhadrosauria, as (informally) defined by Madzia et al. (2020), become

1008 heterodefinitional synonyms. Although such option may still be viewed acceptable, we decided

1009 to apply a minimum-clade definition for Euhadrosauria that makes the name inapplicable under

1010 such hypothesis.

1011

1012 Hadrosauriformes Sereno, 1997 [converted clade name] 
1013 Registration number: 617

1014 Definition. The smallest clade containing Hadrosaurus foulkii Leidy, 1858 and Iguanodon

1015 bernissartensis Boulenger in Beneden, 1881. This is a minimum-clade definition. Abbreviated

1016 definition: min $\nabla$ (Hadrosaurus foulkii Leidy, 1858 \& Iguanodon bernissartensis Boulenger in

1017 Beneden, 1881).

1018 Reference phylogeny. Figure 12 of Madzia et al. (2020) is treated here as the primary reference

1019 phylogeny. Additional reference phylogenies include Figure 20 of Verdú et al. (2018), Figure 3

1020 of Párraga \& Prieto-Márquez (2019), Figure 8 of Słowiak et al. (2020), Figure 11 of McDonald

1021 et al. (2021), and Figure 9 of Verdú et al. (2020).

1022 Composition. Under the primary reference phylogeny, Hadrosauriformes comprises members of 1023 the clades Iguanodontidae and Hadrosauroidea.

1024 Synonyms. If Hypselospinus fittoni nests within the smallest clade containing Hadrosaurus

1025 foulkii and Iguanodon bernissartensis, the name Hadrosauriformes is a potential

1026 heterodefinitional synonym of Neoiguanodontia (see the name entry). In such case, the name

1027 Hadrosauriformes should have priority. The name Iguanodontoidea Hay, 1902 has been also

1028 used as an approximate synonym (Sereno, 1986; Norman, 2002). Note that Norman (2002) used

1029 Iguanodontoidea for a clade "[s]erially more derived than Camptosaurus" (Norman, 2002: 138)

1030 and defined it as "Iguanodon and all iguanodontians more closely related to Edmontosaurus than

1031 to Camptosaurus". Such definition would make Iguanodontoidea applicable for the same clade

1032 as Styracosterna (see the name entry). However, Figure 35 of Norman (2002) shows that the

1033 name does not cover Lurdusaurus, which should be included within the clade under such

1034 maximum-clade definition. Since Norman (2002) considers Iguanodontoidea to be a synonym of

1035 Hadrosauriformes of Sereno (1997, 1998, 1999), it is apparent that Norman's (2002) concept of

1036 Iguanodontoidea would be more similar to that of Hadrosauriformes rather than Styracosterna.

1037 Comments. The name Hadrosauriformes has been (informally) defined before (Sereno, 1998;

1038 Norman, 2015; Madzia et al. 2020). However, only Madzia et al. (2020: Table 1) included the

1039 mandatory $H$. foulkii as the internal specifier. We formalize the definition of Madzia et al.

1040 (2020).

1041

1042 Hadrosaurinae Lambe, 1918 [converted clade name]

1043 Registration number: 618

Peer) reviewing PDF | (2021:04:60693:1:1:NEW 8 Sep 2021) 
1044 Definition. The largest clade containing Hadrosaurus foulkii Leidy, 1858 but not Lambeosaurus 1045 lambei Parks, 1923. This is a maximum-clade definition. Abbreviated definition: max $\nabla$ 1046 (Hadrosaurus foulkii Leidy, 1858 Lambeosaurus lambei Parks, 1923).

1047 Reference phylogeny. Figure 5 of Kobayashi et al. (2019) is treated here as the primary 1048 reference phylogeny. Additional reference phylogenies include Figure 13 of Cruzado-Caballero $1049 \&$ Powell (2017), Figure 20 of Xing et al. (2017), Figure 5 of Zhang et al. (2020), and Figure 10 1050 of Longrich et al. (2021).

1051 Composition. Under the primary reference phylogeny, Hadrosaurinae comprises Hadrosaurus 1052 foulkii and members of the clades Brachylophosaurini, Edmontosaurini, Kritosaurini, and 1053 Saurolophini.

1054 Synonyms. The name Saurolophinae Brown, 1914a has been recently used for the same clade 1055 (under the hypothesis in which H. foulkii is nested outside the smallest clade containing 1056 Saurolophus osborni and Lambeosaurus lambei). See the entry for the name Saurolophinae.

1057 Comments. The name Hadrosaurinae has been (informally) defined before by (Sereno, 1998;

1058 Sereno, 2005). Sereno (1998) applied the maximum-clade definition and used Saurolophus as the 1059 internal specifier and Parasaurolophus as the external specifier. In turn, Sereno (2005), 1060 apparently erroneously, defined Hadrosaurinae as pertaining to "[ $\mathrm{t}$ ]he most inclusive taxon 1061 containing Saurolophus osborni Brown 1912 and Parasaurolophus walkeri Parks 1922 and 1062 including Hadrosaurus foulkii Leidy 1858". Our formal maximum-clade definition was formed 1063 to make Hadrosaurinae applicable regardless of whether the taxon lies ouside or within the 1064 smallest clade containing Saurolophus osborni and Lambeosaurus lambei.

1065

1066 Hadrosauroidea von Huene, 1952 [converted clade name]

1067 Registration number: 619

1068 Definition. The largest clade containing Hadrosaurus foulkii Leidy, 1858 but not Iguanodon 1069 bernissartensis Boulenger in Beneden, 1881. This is a maximum-clade definition. Abbreviated 1070 definition: $\max \nabla$ (Hadrosaurus foulkii Leidy, 1858 Iguanodon bernissartensis Boulenger in 1071 Beneden, 1881).

1072 Reference phylogeny. Figure 12 of Madzia et al. (2020) is treated here as the primary reference 1073 phylogeny. Additional reference phylogenies include Figure 20 of Verdú et al. (2018), Figure 8 
1074 of Słowiak et al. (2020), Figure 11 of McDonald et al. (2021), Figure 11 of Santos-Cubedo et al. 1075 (2021), and Figure 9 of Verdú et al. (2020).

1076 Composition. Under the primary reference phylogeny, Hadrosauroidea comprises Altirhinus

1077 kurzanovi, Batyrosaurus rozhdestvenskyi, Bolong yixianensis, Equijubus normani,

1078 Gongpoquansaurus mazongshanensis, Jinzhousaurus yangi, Koshisaurus katsuyama,

1079 Mantellisaurus atherfieldensis, Morelladon beltrani, Ouranosaurus nigeriensis,

1080 Penelopognathus weishampeli, Proa valdearinnoensis, Probactrosaurus gobiensis,

1081 Ratchasimasaurus suranareae, Sirindhorna khoratensis, Xuwulong yueluni, Zuoyunlong huangi, 1082 and members of the clade Hadrosauromorpha.

1083 Synonyms. No other taxon names are currently in use for the same or approximate clade.

1084 Comments. The name Hadrosauroidea was first (informally) defined by Sereno (1998: 62) who 1085 used the maximum-clade definition and Parasaurolophus walkeri as the internal specifier and

1086 Iguanodon bernissartensis as the external specifier. We formalize the definition of Madzia et al.

1087 (2020: Table 1) who replaced P. walkeri with H. foulkii.

1088

1089 Hadrosauromorpha Norman, 2014 [converted clade name]

1090 Registration number: 620

1091 Definition. The largest clade containing Hadrosaurus foulkii Leidy, 1858 but not

1092 Probactrosaurus gobiensis Rozhdestvensky, 1966. This is a maximum-clade definition.

1093 Abbreviated definition: max $\nabla$ (Hadrosaurus foulkii Leidy, $1858 \sim$ Probactrosaurus gobiensis

1094 Rozhdestvensky, 1966).

1095 Reference phylogeny. Figure 12 of Madzia et al. (2020) is treated here as the primary reference 1096 phylogeny. Additional reference phylogenies include Figure 20 of Verdú et al. (2018), Figure 7 1097 of Kobayashi et al. (2021), Figure 11 of Santos-Cubedo et al. (2021), and Figure 9 of Verdú et al. 1098 (2020).

1099 Composition. Under the primary reference phylogeny, Hadrosauromorpha comprises

1100 Bactrosaurus johnsoni, Datonglong tianzhenensis, Eolambia caroljonesa, Gilmoreosaurus

1101 mongoliensis, Jeyawati rugoculus, Jintasaurus meniscus, Levnesovia transoxiana,

1102 Nanyangosaurus zhugeii, 'Orthomerus dolloi', Plesiohadros djadokhtaensis, Protohadros byrdi,

1103 Tanius sinensis, Tethyshadros insularis, Shuangmiaosaurus gilmorei, Zhanghenglong

1104 yangchengensis, and members of the clade Hadrosauridae.

Peer] reviewing PDF | (2021:04:60693:1:1:NEW 8 Sep 2021) 
1105 Synonyms. No other taxon names are currently in use for the same or approximate clade.

1106 Comments. Hadrosauromorpha was first (informally) defined by Norman (2014: 32) who used

1107 the maximum-clade definition and Parasaurolophus walkeri as the internal specifier and

1108 Probactrosaurus gobiensis as the external specifier. We formalize the definition of Madzia et al.

1109 (2020: Table 1) who replaced P. walkeri with H. foulkii.

1110

1111 Heterodontosauridae Kuhn, 1966 [converted clade name]

1112 Registration number: 622

1113 Definition. The largest clade containing Heterodontosaurus tucki Crompton \& Charig, 1962 but

1114 not Iguanodon bernissartensis Boulenger in Beneden, 1881, Pachycephalosaurus wyomingensis

1115 (Gilmore, 1931), Stegosaurus stenops Marsh, 1887, and Triceratops horridus Marsh, 1889. This

1116 is a maximum-clade definition. Abbreviated definition: max $\nabla$ (Heterodontosaurus tucki

1117 Crompton \& Charig, 1962 Iguanodon bernissartensis Boulenger in Beneden, 1881 \&

1118 Pachycephalosaurus wyomingensis [Gilmore, 1931] \& Stegosaurus stenops Marsh, 1887 \&

1119 Triceratops horridus Marsh, 1889).

1120 Reference phylogeny. Figure 4 of Madzia et al. (2018) is treated here as the primary reference

1121 phylogeny. Additional reference phylogenies include Figure 25 of Herne et al. (2019), Figure 12

1122 of Yang et al. (2020), and Figure 57 of Barta \& Norell (2021).

1123 Composition. Under the primary reference phylogeny, Heterodontosauridae comprises

1124 Abrictosaurus consors, Echinodon becklesii, Eocursor parvus, Fruitadens haagarorum,

1125 Heterodontosaurus tucki, Lycorhinus angustidens, Manidens condorensis, Pegomastax africana, 1126 and Tianyulong confuciusi.

1127 Synonyms. No other taxon names are currently in use for the same or approximate clade.

1128 Comments. We follow Sereno (2012) in recognizing Kuhn (1966), rather than Romer (1966), as

1129 the author establishing Heterodontosauridae. The name Heterodontosauridae has been

1130 (informally) defined before (Sereno, 1998; Sereno, 2005). These definitions were maximum-

1131 clade and used Heterodontosaurus as the internal specifier and Parasaurolophus (Sereno, 1998)

1132 or Parasaurolophus walkeri, Pachycephalosaurus wyomingensis, Triceratops horridus, and

1133 Ankylosaurus magniventris (Sereno, 2005) as the external specifiers. We apply the name

1134 Heterodontosauridae for the same known contents; adopting the mandatory Heterodontosaurus

1135 tucki as the internal specifier and representatives of all major ornithischian lineages, Ceratopsia

PeerJ reviewing PDF | (2021:04:60693:1:1:NEW 8 Sep 2021) 
1136 (Triceratops horridus), Ornithopoda (Iguanodon bernissartensis), Pachycephalosauria

1137 (Pachycephalosaurus wyomingensis), and Thyreophora (Stegosaurus stenops), as the external

1138 specifiers. Note that the external specifiers Pachycephalosaurus wyomingensis, Stegosaurus

1139 stenops, and Triceratops horridus are not included in the primary reference phylogeny. $P$.

1140 wyomingensis and T. horridus belong to Marginocephalia that is indicated on Figure 4 of

1141 Madzia et al. (2018), while S. stenops is nested within Thyreophora (e.g., Maidment et al., 2020).

1142

1143 Huayangosauridae Dong et al., 1982 [converted clade name]

1144 Registration number: 623

1145 Definition. The largest clade containing Huayangosaurus taibaii Dong et al., 1982 but not

1146 Stegosaurus stenops Marsh, 1887. This is a maximum-clade definition. Abbreviated definition:

$1147 \max \nabla$ (Huayangosaurus taibaii Dong et al., 1982 Stegosaurus stenops Marsh, 1887).

1148 Reference phylogeny. Figure 12 of Maidment et al. (2020) is treated here as the primary

1149 reference phylogeny. Additional reference phylogenies include Figure 11 of Maidment et al.

1150 (2008) and Figure 1 of Raven and Maidment (2017).

1151 Composition. Under the primary reference phylogeny, Huayangosauridae comprises

1152 Chungkingosaurus jiangbeiensis and Huayangosaurus taibaii.

1153 Synonyms. No other taxon names are currently in use for the same or approximate clade.

1154 Comments. The name Huayangosauridae was first (informally) defined by Galton and

1155 Upchurch (2004: 358) who used the maximum-clade definition and selected Huayangosaurus as

1156 the internal specifier and Stegosaurus as the external specifier. We formalize this definition.

1157

1158 Hypsilophodontia Cooper, 1985 [converted clade name]

1159 Registration number: 624

1160 Definition. The smallest clade within Ornithopoda containing Hypsilophodon foxii Huxley, 1869

1161 and Tenontosaurus tilletti Ostrom, 1970, provided that it does not include Iguanodon

1162 bernissartensis Boulenger in Beneden, 1881. This is a minimum-clade definition. Abbreviated

1163 definition: $\min \nabla \in$ Ornithopoda (Hypsilophodon foxii Huxley, 1869 \& Tenontosaurus tilletti

1164 Ostrom, $1970 \mid$ Iguanodon bernissartensis Boulenger in Beneden, 1881).

1165 Reference phylogeny. Figure 50 of Norman (2015) is treated here as the primary reference 1166 phylogeny. 
1167 Composition. Under the primary reference phylogeny, Hypsilophodontia comprises a clade

1168 formed by Hypsilophodon foxii, Rhabdodontidae, and Tenontosaurus spp. However, see

1169 'Comments' below for discussion of potential alternative composition of Clypeodonta.

1170 Synonyms. No other taxon names are currently in use for the same or approximate clade.

1171 Comments. The name Hypsilophodontia was (informally) defined as pertaining to

1172 "Hypsilophodon foxii, Tenontosaurus tilletti, their most recent common ancestor, and all of its

1173 descendants" (Norman, 2015: 171). However, such definition does not reflect alternative

1174 topologies that do not show Hypsilophodontia as reconstructed by Norman (2015), making it

1175 applicable for markedly different contents (see, e.g., Madzia et al. 2018: Fig. 4).

1176 Here we define the name Hypsilophodontia using a similar minimum-clade definition as that of

1177 Norman (2015) but by including the part "within Ornithopoda" in the definition, and adding an

1178 external specifier, we restrict the use of Hypsilophodontia to the node only when H. foxii

1179 represents an ornithopod (see Article 11.14 of the ICPN) and when Hypsilophodon foxii and

1180 Tenontosaurus tilletti are more closely related to each other than either is to I. bernissartensis,

1181 following the original intent of Norman (2015). Note that the internal specifier Tenontosaurus

1182 tilletti is not indicated in the primary reference phylogeny. The taxon is the type species of

1183 Tenontosaurus Ostrom, 1970 and is comprised there within the 'tenontosaurs'.

1184

1185

1186

Hypsilophodontidae Dollo, 1882 [converted clade name]

1187

Registration number: 625

1188

Definition. The largest clade containing Hypsilophodon foxii Huxley, 1869 but not Iguanodon

1189 bernissartensis Boulenger in Beneden, 1881 and Rhabdodon priscus Matheron, 1869. This is a maximum-clade definition. Abbreviated definition: $\max \nabla$ (Hypsilophodon foxii Huxley, 1869 Iguanodon bernissartensis Boulenger in Beneden, 1881 \& Rhabdodon priscus Matheron, 1869).

1192 reference phylogeny.

1193 Composition. Under the primary reference phylogeny, Hypsilophodontidae comprises

1194 Hypsilophodon foxii, Gasparinisaura cincosaltensis, and Parksosaurus warreni.

1195 Synonyms. The name Parksosaurinae has been recently for the same contents (Yang et al., 1196 2020), and attributed (apparently following the Principle of Coordination) to Buchholz (2002).

1197 No other taxon names are currently in use for the same or approximate clade. 
1198 Comments. Hypsilophodontidae was first (informally) defined by Sereno (1998: 61) who used

1199 the maximum-clade definition and Hypsilophodon foxii as the internal specifier and

1200 Parasaurolophus walkeri as the external specifier. Here we use the same type of definition but

1201 replace $P$. walkeri with $I$. bernissartensis. This taxon has always been considered outside

1202 Hypsilophodontidae. Additionally, we include Rhabdodon priscus as a second external specifier

1203 to prevent the inclusion of Rhabdodontidae within Hypsilophodontidae under the topology of

1204 Norman (2015: Fig. 50).

1205

1206

Iguanodontia Baur, 1891 [converted clade name]

1207 Registration number: 626

1208 Definition. The smallest clade containing Dryosaurus altus (Marsh, 1878), Iguanodon

1209 bernissartensis Boulenger in Beneden, 1881, Rhabdodon priscus Matheron, 1869, and

1210 Tenontosaurus tilletti Ostrom, 1970, provided that it does not include Hypsilophodon foxii

1211 Huxley, 1869. This is a minimum-clade definition. Abbreviated definition: min $\nabla$ (Dryosaurus

1212 altus [Marsh, 1878] \& Iguanodon bernissartensis Boulenger in Beneden, 1881 \& Rhabdodon

1213 priscus Matheron, 1869 \& Tenontosaurus tilletti Ostrom, $1970 \mid \sim$ Hypsilophodon foxii Huxley, 1214 1869).

1215 Reference phylogeny. Figure 12 of Madzia et al. (2020) is treated here as the primary reference

1216 phylogeny. Additional reference phylogenies include Figure 16 of Han et al. (2018), Figure 20 of

1217 Verdú et al. (2018), Figure 25 of Herne et al. (2019), and Figure 9 of Verdú et al. (2020).

1218 Composition. Under the primary reference phylogeny, Iguanodontia comprises members of the

1219 clade Rhabdodontomorpha, Tenontosaurus spp., and Dryomorpha.

1220 Synonyms. No other taxon names are currently in use for the same or approximate clade.

1221 Clypeodonta, as reconstructed by Norman (2015) covers a similar taxic composition; though the

1222 topology of Norman (2015) differs from that of the primary phylogeny of Iguanodontia

1223 significantly.

1224 Comments. The application of Iguanodontia has been described and discussed by Madzia et al.

1225 (2018: Appendix 1) and Madzia et al. (2020: Table 1). We therefore refer to these studies for

1226 details. Our definition differs from that advocated by Madzia et al. (2018) and Madzia et al.

1227 (2020) in that the name is newly applicable only if it is used for a clade that does not include

1228 Hypsilophodon foxii (e.g., it becomes inapplicable under the topology of Norman, 2015: Fig. 50).

Peer] reviewing PDF | (2021:04:60693:1:1:NEW 8 Sep 2021) 
1230 Iguanodontidae Bonaparte, 1850 [converted clade name]

1231 Registration number: 627

1232 Definition. The largest clade containing Iguanodon bernissartensis Boulenger in Beneden, 1881

1233 but not Hadrosaurus foulkii Leidy, 1858. This is a maximum-clade definition. Abbreviated

1234 definition: $\max \nabla$ (Iguanodon bernissartensis Boulenger in Beneden, $1881 \sim$ Hadrosaurus

1235 foulkii Leidy, 1858).

1236 Reference phylogeny. Figure 13 of Madzia et al. (2020) is treated here as the primary reference

1237 phylogeny. Additional reference phylogenies include Figure 3 of Madzia et al. (2018), Figure 20

1238 of Verdú et al. (2018), Figure 32 of Tsogtbaatar et al. (2019), Figure 7 of Kobayashi et al.

1239 (2021), and Figure 11 of Santos-Cubedo et al. (2021).

1240 Composition. Under the primary reference phylogeny, Iguanodontidae comprises Barilium

1241 dawsoni, Iguanodon bernissartensis, Iguanodon galvensis, and Lurdusaurus arenatus.

1242 Synonyms. The name Iguanodontoidea Hay, 1902 is an approximate synonym of

1243 Iguanodontidae (see, e.g., Figure 20 of Verdú et al., 2018). Both these names have been used for

1244 various sets of taxa thought or reconstructed to be more closely related to Iguanodon

1245 bernissartensis than to hadrosaurids. Considering that significant differences exist between

1246 phylogeny reconstructions of Iguanodon-grade ornithopods (e.g., Madzia et al., 2018; Verdú et

1247 al., 2018; Madzia et al., 2020; McDonald et al., 2021), it is difficult to link either of the names to

1248 a certain, stable composition. Here, we prefer to apply the name Iguanodontidae because it is

1249 more frequent in the literature and because it was coined 52 years before Iguanodontoidea. It is

1250 worth noting that the Iguanodontoidea has been also used as an approximate synonym of

1251 Hadrosauriformes (see the name entry).

1252 Comments. The name Iguanodontidae was first (informally) defined before (Sereno, 1998;

1253 Sereno, 2005; Santos-Cubedo et al., 2021). These definitions were maximum-clade and used

1254 Iguanodon bernissartensis as the internal specifier and Parasaurolophus walkeri (Sereno, 1998;

1255 Sereno, 2005) or Corythosaurus casuarius (Santos-Cubedo et al., 2021) as the external specifier.

1256 We apply a similar definition but replace $P$. walkeri/Corythosaurus casuarius with $H$. foulkii.

1257 Note that even though the study of Santos-Cubedo et al. (2021) appeared after the publication of

1258 Phylonyms (de Queiroz et al., 2020), the work does not meet the general requirements for

1259 establishing Iguanodontidae as a phylogenetically defined clade name (see Articles 7 of the

Peer] reviewing PDF | (2021:04:60693:1:1:NEW 8 Sep 2021) 
$1260 I C P N)$, nor it provides anything that would indicate such intention. Specifically, the name

1261 Iguanodontidae is not explicitly designated as a converted clade name, no bibliographic citations 1262 demonstrating prior application of the name to a taxon approximating the clade for which it is 1263 being established have been provided (including the authorship of the preexisting name), and no 1264 evidence is provided that the required information has been submitted to the registration database 1265 for phylogenetically defined names, the RegNum (registration number is missing). The study 1266 specifies the phylogenetic information, such as the placement of the clade on the ornithopod tree 1267 and the distribution of apomorphies supporting the existence of the clade, and presents the 1268 hypothesized composition of the clade. This information alone, however, would not be sufficient 1269 for the name Iguanodontidae to be established as a converted clade name, as required by the $1270 I C P N$.

1271

1272

1273

Jeholosauridae Han et al., 2012 [converted clade name]

1274 Registration number: 628

1275

Definition. The largest clade outside Hypsilophodontidae or Thescelosauridae containing Jeholosaurus shangyuanensis Xu et al., 2000 but not Hypsilophodon foxii Huxley, 1869, Iguanodon bernissartensis Boulenger in Beneden, 1881, Pachycephalosaurus wyomingensis (Gilmore, 1931), Thescelosaurus neglectus Gilmore, 1913, and Triceratops horridus Marsh, 1889. This is a maximum-clade definition. Abbreviated definition: $\max \nabla \notin$ Hypsilophodontidae V Thescelosauridae (Jeholosaurus shangyuanensis Xu et al., $2000 \sim$ Hypsilophodon foxii Huxley, 1869 \& Iguanodon bernissartensis Boulenger in Beneden, 1881 \& Pachycephalosaurus 1282 horridus Marsh, 1889).

1283 Reference phylogeny. Figure 25 of Herne et al. (2019) is treated here as the primary reference 1284 phylogeny. Additional reference phylogenies include Figure 4 of Madzia et al. (2018), Figure 16 1285 of Han et al. (2018), and Figure 57 of Barta \& Norell (2021).

1286 Composition. Under the primary reference phylogeny, Jeholosauridae comprises

1287 Changchunsaurus parvus, Haya griva, and Jeholosaurus shangyuanensis. Under alternative 1288 hypotheses, however, Jeholosauridae includes Jeholosaurus shangyuanensis and Yueosaurus 1289 tiantaiensis (e.g., Madzia et al., 2018: Fig. 4; Barta \& Norell, 2021: Fig. 57). 
1290 Synonyms. The name Jeholosaurinae has been used recently for the same contents (Yang et al., 1291 2020), and attributed (apparently following the Principle of Coordination) to Han et al. (2012).

1292 No other taxon names are currently in use for the same or approximate clade.

1293 Comments. We use a maximum-clade definition similar to that of Han et al. (2012), which is the 1294 only definition (informally) used for Jeholosauridae. Our definition differs in that we replaced 1295 the original representative of Ceratopsia (Protoceratops andrewsi) with a taxon that is widely 1296 used in phylogenetic definitions of ornithischian clade names (Triceratops horridus).

1297 Additionally, our definition prevents the use of Jeholosauridae under the potential hypotheses in 1298 which Jeholosaurus is inferred as part of Hypsilophodontidae or Thescelosauridae. Note that the 1299 internal specifiers Pachycephalosaurus wyomingensis and Triceratops horridus are not included 1300 in the primary reference phylogeny. The former belongs to Pachycephalosauria (see, e.g., 1301 Dieudonné et al., 2020), while the latter is part of Ceratopsia (e.g., Morschhauser et al., 2019), 1302 both within Marginocephalia that is indicated on Figure 25 of Herne et al. (2019).

\section{Kritosaurini Glut, 1997 [converted clade name]}

1306 Definition. The largest clade containing Kritosaurus navajovius Brown, 1910 but not 1307 Brachylophosaurus canadensis Sternberg, 1953, Edmontosaurus regalis Lambe, 1917, 1308 Hadrosaurus foulkii Leidy, 1858, and Saurolophus osborni Brown, 1912. This is a maximumclade definition. Abbreviated definition: $\max \nabla$ (Kritosaurus navajovius Brown, 1910 Brachylophosaurus canadensis Sternberg, 1953 \& Edmontosaurus regalis Lambe, 1917 \& Hadrosaurus foulkii Leidy, 1858 \& Saurolophus osborni Brown, 1912).

1312 Reference phylogeny. Figure 18 of Prieto-Márquez et al. (2020) is treated here as the primary 1313 reference phylogeny. Additional reference phylogenies include Figure 5 of Kobayashi et al. 1314 (2019), Figure 11 of Prieto-Márquez et al. (2019), Figure 9 of Zhang et al. (2019), Figure 5 of 1315 Zhang et al. (2020), Figure 7 of Kobayashi et al. (2021), and Figure 10 of Longrich et al. (2021). 1316 Composition. Under the primary reference phylogeny Kritosaurini comprises Gryposaurus spp., 1317 Kritosaurus spp., Rhinorex condrupus, Secernosaurus koerneri, and the specimen 'Big Bend 1318 UTEP 37.7'.

1319 Synonyms. No other taxon names are currently in use for the same or approximate clade. 
1320 Comments. The study of Lapparent \& Lavocat (1955) has been cited to be the reference

1321 establishing the name Kritosaurini (e.g., Prieto-Márquez, 2014). However, Lapparent \& Lavocat

1322 (1955) used 'Kritosaurinés' rather than 'Kritosaurini'. The name Kritosaurini was then used by

1323 Brett-Surman (1989) and by Glut (1997). Since Brett-Surman (1989) is an unpublished doctoral

1324 dissertation, we consider Glut (1997) to be the earliest publication to spell the name Kritosaurini.

1325 The name was first (informally) defined by Prieto-Márquez (2014) who applied the minimum-

1326 clade definition and used Kritosaurus navajovius, Gryposaurus notabilis, and Naashoibitosaurus

1327 ostromi as the internal specifiers. We preserve the original intent of Prieto-Márquez (2014) but

1328 prefer to apply the maximum-clade definition. Kritosaurus navajovius is used as the internal

1329 specifier and Hadrosaurus foulkii, and representatives of Brachalophosaurini

1330 (Brachylophosaurus canadensis), Edmontosaurini (Edmontosaurus regalis), and Saurolophini

1331 (Saurolophus osborni), as the external specifiers.

1332

1333 Lambeosaurinae Parks, 1923 [converted clade name]

1334 Registration number: 630

1335 Definition. The largest clade containing Lambeosaurus lambei Parks, 1923 but not Hadrosaurus

1336 foulkii Leidy, 1858 and Saurolophus osborni Brown, 1912. This is a maximum-clade definition.

1337 Abbreviated definition: max $\nabla$ (Lambeosaurus lambei Parks, 1923 Hadrosaurus foulkii Leidy,

13381858 \& Saurolophus osborni Brown, 1912).

1339 Reference phylogeny. Figure 18 of Prieto-Márquez et al. (2020) is treated here as the primary

1340 reference phylogeny. Additional reference phylogenies include Figure 5 of Kobayashi et al.

1341 (2019), Figure 11 of Prieto-Márquez et al. (2019), Figure 9 of Zhang et al. (2019), Figure 5 of

1342 Zhang et al. (2020), Figure 7 of Kobayashi et al. (2021), and Figure 10 of Longrich et al. (2021).

1343 Composition. Under the primary reference phylogeny, Lambeosaurinae comprises Aralosaurus

1344 tuberiferus, Canardia garonnensis, Jaxartosaurus aralensis, and members of the clades

1345 Lambeosaurini, Parasaurolophini, and Tsintaosaurini.

1346 Synonyms. No other taxon names are currently in use for the same or approximate clade.

1347 Comments. The name Lambeosaurinae has been (informally) defined before (Sereno, 1998;

1348 Sereno, 2005; Prieto-Márquez, 2010). These definitions were maximum-clade and used

1349 Parasaurolophus (Sereno, 1998) or Lambeosaurus lambei (Prieto-Márquez, 2010) as the internal

1350 specifiers and Saurolophus (Sereno, 1998) or Hadrosaurus foulkii, Saurolophus osborni, and

Peer] reviewing PDF | (2021:04:60693:1:1:NEW 8 Sep 2021) 
1351 Edmontosaurus regalis (Prieto-Márquez, 2010) as the external specifiers. Sereno (2005),

1352 apparently erroneously, defined Lambeosaurinae as pertaining to "[ $\mathrm{t}]$ he most inclusive taxon 1353 containing Saurolophus osborni Brown 1912 but not Parasaurolophus walkeri Parks 1922 and

1354 including Lambeosaurus lambei Parks 1923”. Our formal maximum-clade definition is similar to 1355 that of Prieto-Márquez (2010) though we have removed E. regalis from the external specifiers 1356 because the taxon is consistently inferred outside Lambeosaurinae (Kobayashi et al., 2019; 1357 Prieto-Márquez et al., 2019; Prieto-Márquez et al., 2020; Zhang et al., 2019; Zhang et al., 2020; 1358 Gates et al., 2021; Kobayashi et al., 2021; Longrich et al., 2021; Ramírez-Velasco et al., 2021). 1359

1360 Lambeosaurini Sullivan et al., 2011 [converted clade name]

1361 Registration number: 631

1362 Definition. The largest clade containing Lambeosaurus lambei Parks, 1923 but not Aralosaurus 1363 tuberiferus Rozhdestvensky, 1968, Parasaurolophus walkeri Parks, 1922, and Tsintaosaurus 1364 spinorhinus Young, 1958. This is a maximum-clade definition. Abbreviated definition: $\max \nabla$ 1365 (Lambeosaurus lambei Parks, 1923 Aralosaurus tuberiferus Rozhdestvensky, 1968 \& 1366 Parasaurolophus walkeri Parks, 1922 \& Tsintaosaurus spinorhinus Young, 1958).

1367 Reference phylogeny. Figure 18 of Prieto-Márquez et al. (2020) is treated here as the primary 1368 reference phylogeny. Additional reference phylogenies include Figure 5 of Kobayashi et al. 1369 (2019), Figure 11 of Prieto-Márquez et al. (2019), Figure 9 of Zhang et al. (2019), Figure 5 of 1370 Zhang et al. (2020), Figure 7 of Kobayashi et al. (2021), and Figure 10 of Longrich et al. (2021).

1371 Composition. Under the primary reference phylogeny, Lambeosaurini comprises Amurosaurus 1372 riabinini, Arenysaurus ardevoli, Blasisaurus canudoi, Corythosaurus spp., Hypacrosaurus 1373 stebingeri, Hypacrosaurus altispinus, Lambeosaurus spp., Magnapaulia laticaudus, Olorotitan 1374 arharensis (misspelled as 'ararhensis' in the primary reference phylogeny), Sahaliyania 1375 elunchunorum, and Velafrons coahuilensis.

1376 Synonyms. The name Corythosaurini Glut, 1997 is an approximate synonym of Lambeosaurini 1377 (e.g., Evans \& Reisz, 2007; Gates et al., 2007; Pereda-Suberbiola et al., 2009). However, its use 1378 has been discouraged (Prieto-Marquéz et al., 2013) and all recent phylogenetic studies preferred 1379 to use Lambeosaurini instead (e.g., Xing et al., 2017; Kobayashi et al., 2019; Prieto-Márquez et 1380 al., 2019; Zhang et al., 2020; Kobayashi et al., 2021; Longrich et al., 2021; Ramírez-Velasco et 1381 al., 2021). No other taxon names are currently in use for the same or approximate clade. 
1382 Comments. Even though Sullivan et al. (2011) did not explicitly formulate the definition of their

1383 newly proposed name Lambeosaurini, they noted that their "definition of the Lambeosaurini

1384 would be equivalent to node 38 of Prieto-Márquez (2010a: fig. 9)” (Sullivan et al., 2011: 417).

1385 The name Lambeosaurini was first (informally) defined by Prieto-Márquez et al. (2013) who

1386 applied the maximum-clade definition and used Lambeosaurus lambei as the internal specifier

1387 and Parasaurolophus walkeri, Tsintaosaurus spinorhinus, and Aralosaurus tuberiferus as the

1388 external specifier. Such defined, the use of Lambeosaurini adheres to the original intent of

1389 Sullivan et al. (2011). We formalize this definition.

1390

1391 Leptoceratopsidae Nopcsa, 1923 [converted clade name]

1392 Registration number: 632

1393 Definition. The largest clade containing Leptoceratops gracilis Brown, 1914b but not

1394 Protoceratops andrewsi Granger \& Gregory, 1923 and Triceratops horridus Marsh, 1889. This

1395 is a maximum-clade definition. Abbreviated definition: max $\nabla$ (Leptoceratops gracilis Brown,

1396 1914b Protoceratops andrewsi Granger \& Gregory, 1923 \& Triceratops horridus Marsh,

1397 1889).

1398 Reference phylogeny. Figure 10 of Morschhauser et al. (2019) is treated here as the primary

1399 reference phylogeny. Additional reference phylogenies include Figure S1 of Knapp et al. (2018),

1400 Figure 8A of Arbour et al. (2019), Figure 3 of Yu et al. (2020), and Figure 4 of Yu et al. (2020).

1401 Composition. Under the primary reference phylogeny, Leptoceratopsidae comprises Cerasinops

1402 hodgskissi, Gryphoceratops morrisoni, Helioceratops brachygnathus, Ischioceratops

1403 zhuchengensis, Koreaceratops hwaseongensis, Leptoceratops gracilis, Montanoceratops

1404 cerorhynchus, Prenoceratops pieganensis, Udanoceratops tchizhovi, Unescoceratops

1405 kopelhusae, and Zhuchengceratops inexpectus.

1406 Synonyms. No other taxon names are currently in use for the same or approximate clade.

1407 Comments. The name Leptoceratopsidae has been (informally) defined before by Makovicky

1408 (2001) who used Leptoceratops gracilis as the internal specifier and Triceratops horridus as the

1409 external specifier. Since Leptoceratopsidae has never been proposed an alternative use, we

1410 formalize a similar definition that differs only in adding Protoceratops andrewsi as a second

1411 external specifier.

1412

Peer] reviewing PDF | (2021:04:60693:1:1:NEW 8 Sep 2021) 
1413 Marginocephalia Sereno, 1986 [converted clade name]

1414 Registration number: 633

1415 Definition. The smallest clade containing Ceratops montanus Marsh, 1888, Pachycephalosaurus

1416 wyomingensis (Gilmore, 1931), and Triceratops horridus Marsh, 1889. This is a minimum-clade

1417 definition. Abbreviated definition: $\min \nabla$ (Ceratops montanus Marsh, 1888 \&

1418 Pachycephalosaurus wyomingensis [Gilmore, 1931] \& Triceratops horridus Marsh, 1889).

1419 Reference phylogeny. Figure 16 of Han et al. (2018) is treated here as the primary reference

1420 phylogeny. Additional reference phylogenies include Figure 4 of Madzia et al. (2018), Figure 25

1421 of Herne et al. (2019), Figure 1 of Dieudonné et al. (2020), Figure 12 of Yang et al. (2020), and

1422 Figure 57 of Barta \& Norell (2021).

1423 Composition. Under the primary reference phylogeny, Marginocephalia comprises members of 1424 the clades Ceratopsia and Pachycephalosauria.

1425 Synonyms. No other taxon names are currently in use for the same or approximate clade.

1426 Comments. The name Marginocephalia has been (informally) defined before (Currie and

1427 Padian, 1997; Sereno, 1998; Sereno, 2005; Madzia et al., 2018; Herne et al., 2019). These

1428 definitions, except for that of Herne et al. (2019), were minimum-clade and used Ceratopsia and

1429 Pachycephalosauria (Currie and Padian, 1997) or Triceratops horridus and Pachycephalosaurus

1430 wyomingensis (Sereno, 1998; Sereno, 2005; Madzia et al., 2018) as the internal specifiers.

1431 Madzia et al. (2018) further included Ceratops montanus as a third internal specifier, stating that

1432 '[t] $]$ he first definition of Marginocephalia was node-based and used 'Ceratopsia' and

1433 'Pachycephalosauria' as the internal specifiers [...]. To follow the definition, and adhere to the

1434 ICPN (Art. 11), we have to use name-bearing species or their type specimens as specifiers which

1435 makes the name to be anchored on the types of Ceratops montanus and Pachycephalosaurus

1436 wyomingensis. Even if C. montanus may be a nomen dubium, its type specimen is unequivocally

1437 nested deeply within Ceratopsia and thus its use does not change the extent of the name"

1438 (Madzia et al., 2018: Appendix 1). In turn, Herne et al. (2019) preferred a maximum-clade

1439 definition with T. horridus and P. wyomingensis as the internal specifiers and Parasaurolophus

1440 walkeri as the external specifier, arguing that "[previous] definitions [were] not complementary

1441 with present definitions of Cerapoda and Ornithopoda within a node-stem triplet arrangement of

1442 clades" and that "re-definition of Marginocephalia as a stem now mirrors its sister stem clade,

1443 Ornithopoda, within a node-based Cerapoda. As a result, this stabilization of definition allows

Peer) reviewing PDF | (2021:04:60693:1:1:NEW 8 Sep 2021) 
1444 for the definitive assignment of all cerapodan OTUs either as ornithopods or marginocephalians" 1445 (Herne et al., 2019: Supplemental Text S1: 4). However, Marginocephalia has never formed 1446 such 'triplet'. Actually, when its use in a 'node-branch triplet' is considered, it is more closely 1447 tied with Ceratopsia and Pachycephalosauria rather than with Cerapoda and Ornithopoda. 1448 Here, the internal specifiers in the definition of Marginocephalia are used from among the taxa 1449 representing the two major subclades - Ceratopsia (Ceratops montanus and Triceratops

1450 horridus) and Pachycephalosauria (Pachycephalosaurus wyomingensis). Note that none of the 1451 internal specifiers is included in the primary reference phylogeny. Ceratops montanus and 1452 Pachycephalosaurus wyomingensis are name-bearers of Ceratopsia and Pachycephalosauria, 1453 respectively, and are deeply dested within these clades (e.g., Mallon et al., 2016; Dieudonné et 1454 al., 2020). Triceratops horridus is a late-diverging member of Chasmosaurinae within 1455 Ceratopsia (e.g., Morschhauser et al., 2019; Fowler \& Freedman Fowler, 2020). 1456

\section{Nasutoceratopsini Ryan et al., 2017 [converted clade name]}

1458 Registration number: 689

1459 Definition. The largest clade containing Nasutoceratops titusi Sampson et al., 2013 but not 1460 Centrosaurus apertus Lambe, 1905. This is a maximum-clade definition. Abbreviated definition: $1461 \max \nabla$ (Nasutoceratops titusi Sampson et al., $2013 \sim$ Centrosaurus apertus Lambe, 1905). 1462 Reference phylogeny. Figure 9 of Chiba et al. (2018) is treated here as the primary reference 1463 phylogeny. Additional reference phylogenies include Figure 7 of Tykoski \& Fiorillo (2012), 1464 Figure 10 of Ryan et al. (2017), and Figure 13 of Dalman et al. (2018).

1465 Composition. Under the primary reference phylogeny, Nasutoceratopsini comprises 1466 Avaceratops lammersi, Nasutoceratops titusi, and the specimens CMN 8804, MOR 692, and the 1467 'Malta New Taxon' (GPDM 63). Under an alternative hypothesis, however, Nasutoceratopsini 1468 includes only a single unequivocal member, Nasutoceratops titusi (Dalman et al., 2021: Fig. 23). 1469 Synonyms. No other taxon names are currently in use for the same or approximate clade. 1470 Comments. The name was first (informally) defined by Ryan et al. (2017) who applied the 1471 maximum-clade definition and used Nasutoceratops titusi as the internal specifier and

1472 Centrosaurus apertus as the external specifier. We formalize this definition.

\section{Neoceratopsia Sereno, 1986 [converted clade name]}


1475 Registration number: 634

1476 Definition. The largest clade containing Triceratops horridus Marsh, 1889 but not

1477 Chaoyangsaurus youngi Zhao et al., 1999 and Psittacosaurus mongoliensis Osborn, 1923. This

1478 is a maximum-clade definition. Abbreviated definition: max $\nabla$ (Triceratops horridus Marsh,

$14791889 \sim$ Chaoyangsaurus youngi Zhao et al., 1999 \& Psittacosaurus mongoliensis Osborn, 1923).

1480 Reference phylogeny. Figure 10 of Morschhauser et al. (2019) is treated here as the primary

1481 reference phylogeny. Additional reference phylogenies include Figure 16 of Han et al. (2018),

1482 Figure S1 of Knapp et al. (2018), and Figure 4 of Yu et al. (2020).

1483 Composition. Under the primary reference phylogeny, Neoceratopsia comprises Aquilops

1484 americanus, Archaeoceratops oshimai, Asiaceratops salsopaludalis, Auroraceratops rugosus,

1485 ZPAL MgD-I/156 (= Graciliceratops mongoliensis), Liaoceratops yanzigouensis, Mosaiceratops

1486 azumai, Stenopelix valdensis, Yamaceratops dorngobiensis, and members of the clades

1487 Leptoceratopsidae and Coronosauria.

1488 Synonyms. No other taxon names are currently in use for the same or approximate clade.

1489 Comments. The name Neoceratopsia has been (informally) defined before by Sereno (1998,

1490 2005) who applied a maximum-clade definition and used Triceratops horridus as the internal

1491 specifier and Psittacosaurus mongoliensis as the external specifiers. We further include a second 1492 external specifier, Chaoyangsaurus youngi, to ensure that Chaoyangsauridae, a clade usually 1493 reconstructed as some of the earliest-diverging ceratopsians (e.g., Han et al., 2018; Knapp et al., 1494 2018; Yu et al., 2020), are maintained outside Neoceratopsia.

1495

1496 Neoiguanodontia Norman, 2014 [converted clade name]

1497 Registration number: 635

1498 Definition. The smallest clade containing Hypselospinus fittoni (Lydekker, 1889), Iguanodon

1499 bernissartensis Boulenger in Beneden, 1881, and Parasaurolophus walkeri Parks 1922. This is a

1500 minimum-clade definition. Abbreviated definition: $\min \nabla$ (Hypselospinus fittoni [Lydekker,

1501 1889] \& Iguanodon bernissartensis Boulenger in Beneden, 1881 \& Parasaurolophus walkeri

1502 Parks 1922).

1503 Reference phylogeny. Figure 2.26 of Norman (2014) is treated here as the primary reference 1504 phylogeny. Additional reference phylogenies include Figure 50 of Norman (2015), Figure 3 of 1505 Párraga \& Prieto-Márquez (2019), and Figure 11 of McDonald et al. (2021). 
1506 Composition. Under the primary reference phylogeny, Neoiguanodontia comprises

1507 Hypselospinus fittoni and members of the clades Iguanodontidae and Hadrosauroidea.

1508 Synonyms. Neoiguanodontia is a potential heterodefinitional synonym of Hadrosauriformes. If

1509 Hypselospinus fittoni nests within the smallest clade containing Hadrosaurus foulkii and

1510 Iguanodon bernissartensis (e.g., Verdú et al., 2018; Santos-Cubedo et al., 2021: Fig. 11), the

1511 name Hadrosauriformes should have priority.

1512 Comments. The application of Neoiguanodontia has been described and discussed by Madzia et 1513 al. (2020: Table 1). We therefore refer to that study for details.

1514

1515 Neornithischia Cooper, 1985 [converted clade name]

1516 Registration number: 636

1517 Definition. The largest clade containing Iguanodon bernissartensis Boulenger in Beneden, 1881

1518 and Triceratops horridus Marsh, 1889 but not Ankylosaurus magniventris Brown, 1908 and

1519 Stegosaurus stenops Marsh, 1887. This is a maximum-clade definition. Abbreviated definition:

$1520 \max \nabla$ (Iguanodon bernissartensis Boulenger in Beneden, 1881 \& Triceratops horridus Marsh,

15211889 Ankylosaurus magniventris Brown, 1908 \& Stegosaurus stenops Marsh, 1887).

1522 Reference phylogeny. Figure 4 of Madzia et al. (2018) is treated here as the primary reference

1523 phylogeny. Additional reference phylogenies include Figure 16 of Han et al. (2018), Figure 25 of

1524 Herne et al. (2019), Figure 1 of Dieudonné et al. (2020), and Figure 57 of Barta \& Norell (2021).

1525 Composition. Under the primary reference phylogeny, Neornithischia comprises Agilisaurus

1526 louderbacki, Hexinlusaurus multidens, Hypsilophodon foxii, Kulindadromeus zabaikalicus,

1527 Leaellynasaura amicagraphica, Lesothosaurus diagnosticus, Othnielosaurus consors (=

1528 Nanosaurus agilis; see Carpenter \& Galton, 2018) Yandusaurus hongheensis, and members of

1529 the clades Cerapoda, Jeholosauridae, and Thescelosauridae.

1530 Synonyms. No other taxon names are currently in use for the same or approximate clade.

1531 Comments. The name Neornithischia has been (informally) defined before (Sereno, 1998;

1532 Sereno, 2005; Butler et al., 2008; Herne et al., 2019). These definitions were maximum-clade

1533 and used Triceratops horridus (Sereno, 1998), Parasaurolophus walkeri (Butler et al., 2008) or

1534 both, T. horridus and P. walkeri (Sereno, 2005; Herne et al., 2019) as the internal specifiers, and

1535 Ankylosaurus magniventris (Sereno, 1998; Sereno, 2005; Herne et al., 2019) or A. magniventris

1536 and Stegosaurus stenops (Butler et al., 2008) as the external specifiers. In order to maintain the 
1537 'traditional' concept of Genasauria as a clade comprising Neornithischia and Thyreophora, the

1538 internal specifiers in the definition of Neornithischia are used from among the taxa representing

1539 the two major subclades - Ornithopoda (Iguanodon bernissartensis) and Marginocephalia

1540 (Triceratops horridus) - and the external specifiers are used from among the taxa representing

1541 the thyreophoran clades Ankylosauria (Ankylosaurus magniventris) and Stegosauria

1542 (Stegosaurus stenops). Note that the internal specifier Triceratops horridus and the external

1543 specifiers Ankylosaurus magniventris and Stegosaurus stenops are not included in the primary

1544 reference phylogeny. T. horridus belongs to Ceratopsia (see, e.g., Morschhauser et al., 2019),

1545 while Ankylosaurus magniventris and Stegosaurus stenops are deeply nested members of

1546 Thyreophora (e.g., Thompson et al., 2012; Maidment et al. 2020), a clade that is indicated on

1547 Figure 4 of Madzia et al. (2018).

1548

1549 Nodosauridae Marsh, 1890 [converted clade name]

1550 Registration number: 637

1551 Definition. The largest clade containing Nodosaurus textilis Marsh, 1889 but not Ankylosaurus

1552 magniventris Brown, 1908. This is a maximum-clade definition. Abbreviated definition: $\max \nabla$

1553 (Nodosaurus textilis Marsh, 1889 Ankylosaurus magniventris Brown, 1908).

1554 Reference phylogeny. Figure 5 of Rivera-Sylva et al. (2018a) is treated here as the primary

1555 reference phylogeny. Additional reference phylogenies include Figure 3 of Thompson et al.

1556 (2012), Figure 11 of Arbour and Currie (2016), Figure 1 of Arbour et al. (2016), and Figure 3 of

1557 Brown et al. (2017).

1558 Composition. Under the primary reference phylogeny, Nodosauridae comprises

1559 Dongyangopelta yangyanensis, Gastonia burgei, Gargoyleosaurus parkpinorum, and members

1560 of the clades Nodosaurinae and Polacanthinae.

1561 Synonyms. No other taxon names are currently in use for the same or approximate clade.

1562 Comments. The name Nodosauridae has been (informally) defined before by Sereno (1998,

1563 2005) who used Panoplosaurus mirus (Sereno, 1998) or P. mirus and Nodosaurus textilis Sereno

1564 (2005) as the internal specifiers and Ankylosaurus magniventris as the external specifier.

1565 Considering that all phylogeny reconstructions that include $P$. mirus and $N$. textilis indicate that

1566 these taxa are more closely related to each other than either is to A. magniventris (or placed

Peer] reviewing PDF | (2021:04:60693:1:1:NEW 8 Sep 2021) 
1567 outside the Ankylosauridae + Nodosauridae node), we use a definition that incorporates

1568 Nodosaurus textilis as the sole internal specifier.

1569

1570 Nodosaurinae Abel, 1919 [converted clade name]

1571 Registration number: 638

1572 Definition. The largest clade containing Nodosaurus textilis Marsh, 1889, but not Hylaeosaurus

1573 armatus Mantell, 1833, Mymoorapelta maysi Kirkland \& Carpenter, 1994, and Polacanthus foxii

1574 Owen in Anonymous, 1865. This is a maximum-clade definition. Abbreviated definition: $\max \nabla$

1575 (Nodosaurus textilis Marsh, 1889 Hylaeosaurus armatus Mantell, 1833 \& Mymoorapelta

1576 maysi Kirkland \& Carpenter, 1994 \& Polacanthus foxii Owen in Anonymous, 1865).

1577 Reference phylogeny. Figure 5 of Rivera-Sylva et al. (2018a) is treated here as the primary 1578 reference phylogeny. Additional reference phylogenies include Figure 3 of Thompson et al.

1579 (2012), Figure 11 of Arbour and Currie (2016), Figure 1 of Arbour et al. (2016), and Figure 3 of 1580 Brown et al. (2017).

1581 Composition. Under the primary reference phylogeny, Nodosaurinae comprises Acantholipan

1582 gonzalezi, Ahshislepelta minor, Niobrarasaurus coleii, Nodosaurus textilis, Peloroplites

1583 cedrimontanus, Sauropelta edwardsi, Silvisaurus condrayi, Taohelong jinchengensis,

1584 Tatankacephalus cooneyorum, members of the clades Panoplosaurini and Struthiosaurini, and

1585 the specimen CPC 273.

1586 Synonyms. No other taxon names are currently in use for the same or approximate clade.

1587 Comments. The name Nodosaurinae has been (informally) defined before (Sereno, 1998;

1588 Sereno, 2005). Both these definitions were maximum-clade and used Panoplosaurus (Sereno,

1589 1998) or Panoplosaurus mirus and Nodosaurus textilis (Sereno, 2005) as the internal specifiers

1590 and Sarcolestes and Hylaeosaurus (Sereno, 1998) or Polacanthus foxii, Hylaeosaurus armatus,

1591 and Mymoorapelta maysi as the external specifiers (Sereno, 2005). We formalize a definition

1592 similar to that of Sereno (2005) but use a single internal specifier.

1593

1594 Ornithischia Seeley, 1888 [converted clade name]

1595 Registration number: 639

1596 Definition. The largest clade containing Iguanodon bernissartensis Boulenger in Beneden, 1881

1597 but not Allosaurus fragilis Marsh, 1877a and Camarasaurus supremus Cope, 1877. This is a

Peer] reviewing PDF | (2021:04:60693:1:1:NEW 8 Sep 2021) 
1598 maximum-clade definition. Abbreviated definition: $\max \nabla$ (Iguanodon bernissartensis Boulenger

1599 in Beneden, 1881 Allosaurus fragilis Marsh, 1877a \& Camarasaurus supremus Cope, 1877).

1600 Reference phylogeny. Figure 4 of Madzia et al. (2018) is treated here as the primary reference

1601 phylogeny. Additional reference phylogenies include Figure 2 of Boyd (2015), Figure 1 of Baron

1602 et al. (2017a), Figure 1 of Baron et al. (2017b), and Figure 1 of Langer et al. (2017).

1603 Composition. Under the primary reference phylogeny, Ornithischia comprises Pisanosaurus

1604 mertii and members of the clades Heterodontosauridae and Genasauria. Note, however, that the

1605 early evolution and basal branching of Ornithischia is currently unsettled. For example, $P$.

1606 mertii, may represent either an early-diverging ornithischian (recently, e.g., Desojo et al. 2020)

1607 or a (non-dinosaur) silesaurid dinosauriform (Agnolín \& Rozadilla 2018, Baron 2019). The same

1608 may be true for members of Silesauridae, a group often reconstructed as the sister taxon to

1609 Dinosauria (e.g., Nesbitt et al., 2010; Peecook et al., 2013; Ezcurra, 2016; Cau, 2018; Ezcurra et

1610 al., 2020), that have recently been inferred to represent early-diverging representatives of

1611 Ornithischia (Langer \& Ferigolo, 2013; Cabreira et al., 2016; Pacheco et al., 2019; Müller \&

1612 Garcia, 2020).

1613 Synonyms. No other taxon names are currently in use for the same or approximate clade.

1614 Though not in use, the name Predentata Marsh, 1894 is being occasionally recalled as an

1615 approximate synonym (e.g., Butler et al., 2008; Langer \& Ferigolo, 2013).

1616 Comments. The name Ornithischia has been (informally) defined before (Padian \& May, 1993;

1617 Sereno, 1998; Weishampel, 2004; Norman et al., 2004a; Sereno, 2005; Baron et al., 2017a).

1618 These definitions were maximum-clade and used Triceratops horridus (Padian \& May, 1993;

1619 Sereno, 1998; Weishampel, 2004; Sereno, 2005; Baron et al., 2017a) or Iguanodon

1620 bernissartensis (Norman et al., 2004a) as the internal specifiers. In turn, "birds" (Padian \& May,

1621 1993), Neornithes (Sereno, 1998), Tyrannosaurus (Weishampel, 2004), Cetiosaurus (Norman et

1622 al., 2004a), Passer domesticus and Saltasaurus loricatus (Sereno, 2005), and Passer domesticus

1623 and Diplodocus carnegii (Baron et al., 2017a) were used as the external specifiers. Although

1624 both, I. bernissartensis and T. horridus, are clearly 'traditional' members of Ornithischia, we

1625 have selected the former as the internal specifier and Allosaurus fragilis and Camarasaurus

1626 supremus as the external specifiers. These specifiers are preferred because (a) they represent

1627 deeply nested taxa within their respective clades (Ornithischia, Theropoda, and

1628 Sauropodomorpha), (b) they have been historically associated with these clades, thus being their 
1629 'traditional' members, and (c) their phylogenetic placements are stable across studies. Two 1630 external specifiers, instead of one, are used due to the alternative topologies of dinosaur 1631 relationships (see, e.g., Baron et al., 2017a; Langer et al., 2017). Additionally, Iguanodon 1632 bernissartensis was used as the internal specifier in the formal definition of Dinosauria (Langer 1633 et al., 2020), considered therein as a 'traditional' representative of Ornithischia, and the external 1634 specifier in the formal definition of Sauropodomorpha (Fabbri et al., 2020), again considered 1635 therein as a 'traditional' representative of Ornithischia; A. fragilis was used as the internal 1636 specifier in the formal definitions of Theropoda (Naish et al. 2020) and Saurischia (Gauthier et 1637 al., 2020), and as the external specifier in the formal definition of Sauropodomorpha (Fabbri et 1638 al., 2020), considered in the latter two contributions as a 'traditional' representative of 1639 Theropoda; and Camarasaurus supremus was used as the internal specifier in the formal 1640 definition of Saurischia (Gauthier et al., 2020) and considered therein as a 'traditional' 1641 representative of Sauropodomorpha.

1642

1643

Ornithopoda Marsh, 1881 [converted clade name]

1644 Registration number: 640

1645 Definition. The largest clade containing Iguanodon bernissartensis Boulenger in Beneden, 1881 1646 but not Pachycephalosaurus wyomingensis (Gilmore, 1931) and Triceratops horridus Marsh, 1889. This is a maximum-clade definition. Abbreviated definition: $\max \nabla$ (Iguanodon bernissartensis Boulenger in Beneden, 1881 Pachycephalosaurus wyomingensis [Gilmore, 1931] \& Triceratops horridus Marsh, 1889).

Reference phylogeny. Figure 4 of Madzia et al. (2018) is treated here as the primary reference phylogeny. Additional reference phylogenies include Figure 16 of Han et al. (2018), Figure 25 of Herne et al. (2019), Figure 1 of Dieudonné et al. (2020), and Figure 57 of Barta \& Norell (2021). augustai, Gideonmantellia amosanjuanae, and members of the clades Elasmaria and Iguanodontia.

1656 Synonyms. No other taxon names are currently in use for the same or approximate clade.

1657 Comments. The name Ornithopoda has been (informally) defined before (Sereno, 1998; 1658 Norman et al., 2004b; Sereno, 2005; Butler et al. 2008; Herne et al. 2019). Two of these 1659 definitions were minimum-clade (Sereno, 1998; Sereno, 2005) and used Parasaurolophus 
1660 walkeri and Heterodontosaurus tucki as the internal specifiers. Sereno (2005) further restricted

1661 the name to a hypothesis in which $P$. walkeri and $H$. tucki were more closely related to each

1662 other than either was to Pachycephalosaurus wyomingensis, Triceratops horridus, and

1663 Ankylosaurus magniventris. In turn, Norman et al. (2004b), Butler et al. (2008), and Herne et al.

1664 (2019) defined Ornithopoda as pertaining to the largest clade containing Edmontosaurus regalis

1665 (in Norman et al., 2004b) or P. walkeri (in Butler et al., 2008 and Herne et al., 2019) but not $T$.

1666 horridus. Herne et al. (2019) additionally included a second external specifier (P. wyomingensis).

1667 We selected a definition that follows Herne et al. (2019) in that it includes two external specifiers

1668 (T. horridus and P. wyomingensis, representatives of two clades closely related to ornithopods;

1669 i.e., Ceratopsia and Pachycephalosauria, respectively). However, we prefer to use Iguanodon

1670 bernissartensis as the internal specifier rather than $P$. walkeri, because the former is among the

1671 few taxa that have been considered a part of Ornithopoda when the name was being introduced

1672 in the literature (e.g., Marsh, 1882). The inclusion of a different internal specifier does not

1673 change the extent of Ornithopoda under any of the published phylogeny inferences. Note that the

1674 external specifiers Pachycephalosaurus wyomingensis and Triceratops horridus are not included

1675 in the primary reference phylogeny. The former belongs to Pachycephalosauria (see, e.g.,

1676 Dieudonné et al., 2020), while the latter is part of Ceratopsia (e.g., Morschhauser et al., 2019),

1677 both within Marginocephalia that is indicated on Figure 4 of Madzia et al. (2018).

1678

1679 Orodrominae Brown et al., 2013 [converted clade name]

1680 Registration number: 641

1681 Definition. The largest clade within Hypsilophodontidae $\vee$ Thescelosauridae containing

1682 Orodromeus makelai Horner \& Weishampel, 1988 but not Hypsilophodon foxii Huxley, 1869

1683 and Thescelosaurus neglectus Gilmore, 1913. This is a maximum-clade definition. Abbreviated

1684 definition: $\max \nabla \in$ Hypsilophodontidae $\vee$ Thescelosauridae (Orodromeus makelai Horner \&

1685 Weishampel, 1988 Hypsilophodon foxii Huxley, 1869 \& Thescelosaurus neglectus Gilmore, 1686 1913).

1687 Reference phylogeny. Figure 4 of Madzia et al. (2018) is treated here as the primary reference 1688 phylogeny. Additional reference phylogenies include Figure 25 of Herne et al. (2019) and Figure 168957 of Barta \& Norell (2021). 
1690 Composition. Under the primary reference phylogeny, Orodrominae comprises Albertadromeus 1691 syntarsus, Changchunsaurus parvus, Haya griva, Koreanosaurus boseongensis, Orodromeus 1692 makelai, Oryctodromeus cubicularis, Zephyrosaurus schaffi, and the 'Kaiparowits orodromine'.

1693 Synonyms. No other taxon names are currently in use for the same or approximate clade.

1694 Comments. The name Orodrominae has been (informally) defined before (Brown et al., 2013;

1695 Boyd, 2015). Both these definitions were maximum-clade and used Orodromeus makelai as the 1696 internal specifier and Thescelosaurus neglectus (Brown et al., 2013) or Thescelosaurus neglectus 1697 and Parasaurolophus walkeri (Boyd, 2015) as the external specifiers. Considering the

1698 'traditional concept' of Orodrominae, as a subclade of Thescelosauridae/'hypsilophodonts', and 1699 keeping in mind the unstable phylogenetic position of $H$. foxii (e.g., Madzia et al., 2018), we 1700 apply Orodrominae only when it is inferred either within Thescelosauridae or

1701 Hypsilophodontidae (see Article 11.14 of the ICPN).

1702

1703

1704

Pachycephalosauria Maryańska \& Osmólska, 1974 [converted clade name]

1705

1706

1707

1708

1709

1710

1711

1712

1713

1714

1715

1716

1717

1718

1719

1720

\section{Registration number: 642}

Definition. The largest clade containing Pachycephalosaurus wyomingensis (Gilmore, 1931) but not Ceratops montanus Marsh, 1888 and Triceratops horridus Marsh, 1889. This is a maximumclade definition. Abbreviated definition: max $\nabla$ (Pachycephalosaurus wyomingensis [Gilmore, 1931] Ceratops montanus Marsh, 1888 \& Triceratops horridus Marsh, 1889).

Reference phylogeny. Figure 27 of Schott \& Evans (2017) is treated here as the primary reference phylogeny. Additional reference phylogenies include Figure 5 of Evans et al. (2013), Figure 16 of Han et al. (2018), and Figure 1 of Dieudonné et al. (2020).

Composition. Under the primary reference phylogeny, Pachycephalosauria comprises Wannanosaurus yanshiensis and members of the clade Pachycephalosauridae.

Synonyms. No other taxon names are currently in use for the same or approximate clade.

Comments. The name Pachycephalosauria has been (informally) defined before (Sereno, 1998;

Maryańska et al., 2004; Sereno, 2005). These definitions were maximum-clade and used

Pachycephalosaurus (Sereno, 1998) or Pachycephalosaurus wyomingensis (Maryańska et al., 2004; Sereno, 2005) as the internal specifiers and Triceratops (Sereno, 1998), Triceratops horridus (Maryańska et al., 2004), or Triceratops horridus, Heterodontosaurus tucki, Hypsilophodon foxii, and Ankylosaurus magniventris (Sereno, 2005) as the external specifiers.

Peer] reviewing PDF | (2021:04:60693:1:1:NEW 8 Sep 2021) 
1721 Even though the position of Hypsilophodon foxii and Heterodontosaurus tucki is unstable across

1722 studies (e.g., see, e.g., Han et al., 2018; Madzia et al., 2018; Herne et al., 2019; Dieudonné et al.,

1723 2020), and, for example, Heterodontosauridae were inferred to be more closely related to $P$.

1724 wyomingensis than to T. horridus (Dieudonné et al., 2020: Fig. 1), inclusion of these taxa among

1725 the external specifiers does not need to be necessary as it can be expected that

1726 Pachycephalosauria, as traditionally defined, may cover taxa that are markedly different from

1727 the Late Cretaceous members of the clade. We use a definition similar to that of Maryańska et al.

1728 (2004) but include Ceratops montanus as a second external specifier. Note that none of the

1729 external specifiers is included in the primary reference phylogeny. Both, C. montanus and T.

1730 horridus, however, are members of Ceratopsidae within Ceratopsia (e.g., Mallon et al., 2016;

1731 Morschhauser et al., 2019).

1732

1733 Pachycephalosauridae Sternberg, 1945 [converted clade name]

1734 Registration number: 643

1735 Definition. The smallest clade containing Pachycephalosaurus wyomingensis (Gilmore, 1931)

1736 and Stegoceras validum Lambe, 1902, provided that it does not include Heterodontosaurus tucki

1737 Crompton and Charig, 1962. This is a minimum-clade definition. Abbreviated definition: min $\nabla$

1738 (Pachycephalosaurus wyomingensis [Gilmore, 1931] \& Stegoceras validum Lambe, 1902 |

1739 Heterodontosaurus tucki Crompton and Charig, 1962).

1740 Reference phylogeny. Figure 27 of Schott \& Evans (2017) is treated here as the primary

1741 reference phylogeny. Additional reference phylogenies include Figure 5 of Evans et al. (2013),

1742 Figure 3 of Williamson \& Brusatte (2016), and Figure 14 of Woodruff et al. (2021).

1743 Composition. Under the primary reference phylogeny, Pachycephalosauridae comprises

1744 Colepiocephale lambei, Hanssuesia sternbergi, Stegoceras spp., and members of the clade

1745 Pachycephalosaurinae.

1746 Synonyms. No other taxon names are currently in use for the same or approximate clade.

1747 Comments. The name Pachycephalosauridae has been (informally) defined before by Sereno

1748 (1998, 2005) who applied the minimum-clade definition and used Pachycephalosaurus

1749 wyomingensis and Stegoceras validum as the internal specifiers. This definition is followed here

1750 though we also include a qualifying clause that excludes $H$. tucki from Pachycephalosauridae.

1751 Even though no phylogenetic analysis has ever reconstructed H. tucki or any other 'traditional'

Peer] reviewing PDF | (2021:04:60693:1:1:NEW 8 Sep 2021) 
1752 heterodontosaurid to be within the smallest clade containing P. wyomingensis and S. validum,

1753 Heterodontosauridae were inferred to be early-diverging pachycephalosaurs (Dieudonné et al.,

1754 2020). The addition of a qualifying clause that excludes H. tucki from Pachycephalosauridae

1755 will therefore ensure that the name will never comprise Heterodontosauridae. Note that $H$. tucki

1756 is not included in the primary reference phylogeny. See Figure 1 of Dieudonné et al. (2020) for

1757 its potential placement with respect to pachycephalosaurids.

1758

1759 Pachycephalosaurinae Sereno, 1997 [converted clade name]

1760 Registration number: 748

1761 Definition. The largest clade containing Pachycephalosaurus wyomingensis (Gilmore, 1931) but

1762 not Stegoceras validum Lambe, 1902. This is a maximum-clade definition. Abbreviated

1763 definition: $\max \nabla$ (Pachycephalosaurus wyomingensis [Gilmore, 1931] Stegoceras validum

1764 Lambe, 1902).

1765 Reference phylogeny. Figure 27 of Schott \& Evans (2017) is treated here as the primary

1766 reference phylogeny. Additional reference phylogenies include Figure 7 of Longrich et al.

1767 (2010), Figure 5 of Evans et al. (2013), Figure 3 of Williamson \& Brusatte (2016), and Figure 14 1768 of Woodruff et al. (2021).

1769 Composition. Under the primary reference phylogeny, Pachycephalosaurinae comprises

1770 Acrotholus audeti, Amtocephale gobiensis, Foraminacephale brevis, Goyocephale lattimorei,

1771 Homalocephale calathocercos, Prenocephale prenes, Sphaerotholus spp., Tylocephale gilmorei,

1772 and members of the clade Pachycephalosaurini.

1773 Synonyms. No other taxon names are currently in use for the same or approximate clade.

1774 Comments. The name Pachycephalosaurinae has been (informally) defined before (Sereno,

1775 1998; Sullivan, 2003; Sereno, 2005). Both types of definitions, minimum-clade as well as

1776 maximum-clade, have been proposed for the name. Sereno $(1998,2005)$ preferred a maximum-

1777 clade definition and used Pachycephalosaurus (Sereno, 1998) or Pachycephalosaurus

1778 wyomingensis (Sereno, 2005) as the internal specifier and Stegoceras (Sereno, 1998) or

1779 Stegoceras validum (Sereno, 2005) as the external specifier, while Sullivan (2003) applied a

1780 minimum-clade definition, using Colepiocephale, Prenocephale, Tylocephale, Hanssuesia, and

1781 Pachycephalosaurus and Stygimoloch (Pachycephalosaurini sensu Sullivan, 2003), as the

1782 internal specifiers. We formalize the definition of Sereno (2005).

Peer) reviewing PDF | (2021:04:60693:1:1:NEW 8 Sep 2021) 
1783

1784 Pachycephalosaurini Sullivan, 2003 [converted clade name]

1785 Registration number: 749

1786 Definition. The largest clade containing Pachycephalosaurus wyomingensis (Gilmore, 1931) but

1787 not Prenocephale prenes Maryańska \& Osmólska, 1974 and Sphaerotholus goodwini

1788 Williamson \& Carr, 2003. This is a maximum-clade definition. Abbreviated definition: $\max \nabla$

1789 (Pachycephalosaurus wyomingensis [Gilmore, 1931] Prenocephale prenes Maryańska \&

1790 Osmólska, 1974 \& Sphaerotholus goodwini Williamson \& Carr, 2003).

1791 Reference phylogeny. Figure 27 of Schott \& Evans (2017) is treated here as the primary

1792 reference phylogeny. Additional reference phylogenies include Figure 7 of Longrich et al.

1793 (2010), Figure 5 of Evans et al. (2013), Figure 3 of Williamson \& Brusatte (2016), and Figure 14

1794 of Woodruff et al. (2021).

1795 Composition. Under the primary reference phylogeny, Pachycephalosaurini comprises

1796 Alaskacephale gongloffi, Dracorex hogwartsia, Pachycephalosaurus wyomingensis, and

1797 Stygimoloch spinifer.

1798 Synonyms. No other taxon names are currently in use for the same or approximate clade.

1799 Comments. The name Pachycephalosaurini has been (informally) defined before by Sereno

1800 (2005) who applied a minimum-clade definition and used Pachycephalosaurus wyomingensis

1801 and Stygimoloch spinifer as the internal specifiers. Even though such definition is congruent with

1802 the original intent of Sullivan (2003) who established the taxon name to unite $S$. spinifer and $P$.

1803 wyomingensis, it has since been hypothesized that $S$. spinifer and $P$. wyomingensis may represent

1804 different growth stages of a single taxon (P. wyomingensis) rather than two distinct taxa (Horner

1805 \& Goodwin, 2009). Nevertheless, the name Pachycephalosaurini may still be considered useful

1806 as recent studies indicate close relationships between $P$. wyomingensis and Alaskacephale

1807 gangloffi (Longrich et al., 2010; Evans et al., 2013; Williamson \& Brusatte, 2016; Schott \&

1808 Evans, 2017; Woodruff et al., 2021). Owing to the unsettled phylogenetic ties between the latest

1809 Cretaceous pachycephalosaurs, we prefer to establish a maximum-clade definition for

1810 Pachycephalosaurini to enable the name to be used for a wider range of late-diverging members

1811 of Pachycephalosauridae.

1812

1813 Pachyrhinosaurini Fiorillo \& Tykoski, 2012 [converted clade name]

Peer] reviewing PDF | (2021:04:60693:1:1:NEW 8 Sep 2021) 
1814 Registration number: 690

1815 Definition. The largest clade containing Pachyrhinosaurus canadensis Sternberg, 1950 but not

1816 Centrosaurus apertus Lambe, 1905. This is a maximum-clade definition. Abbreviated definition:

1817 max $\nabla$ (Pachyrhinosaurus canadensis Sternberg, $1950 \sim$ Centrosaurus apertus Lambe, 1905).

1818 Reference phylogeny. Figure 9 of Chiba et al. (2018) is treated here as the primary reference

1819 phylogeny. Additional reference phylogenies include Figure 7 of Tykoski \& Fiorillo (2012),

1820 Figure 10 of Ryan et al. (2017), Figure 13 of Dalman et al. (2018), Figure 10 of Wilson et al.

1821 (2020), and Figure 23 of Dalman et al. (2021).

1822 Composition. Under the primary reference phylogeny, Pachyrhinosaurini comprises

1823 Einiosaurus procurvicornis and members of the clade Pachyrostra.

1824 Synonyms. No other taxon names are currently in use for the same or approximate clade.

1825 Comments. The name was first (informally) defined by Fiorillo \& Tykoski (2012) who applied

1826 the maximum-clade definition and used Pachyrhinosaurus canadensis as the internal specifier

1827 and Centrosaurus apertus as the external specifier. We formalize this definition.

1828

1829

1830 Registration number: 691

1831 Definition. The smallest clade containing Achelousaurus horneri Sampson, 1995 and

Pachyrostra Fiorillo \& Tykoski, 2012 [converted clade name]

1832 Pachyrhinosaurus canadensis Sternberg, 1950. This is a minimum-clade definition. Abbreviated

1833 definition: $\min \nabla$ (Achelousaurus horneri Sampson, 1995 \& Pachyrhinosaurus canadensis

1834 Sternberg, 1950).

1835 Reference phylogeny. Figure 9 of Chiba et al. (2018) is treated here as the primary reference

1836 phylogeny. Additional reference phylogenies include Figure 7 of Tykoski \& Fiorillo (2012),

1837 Figure 10 of Ryan et al. (2017), Figure 13 of Dalman et al. (2018), Figure 10 of Wilson et al.

1838 (2020), and Figure 23 of Dalman et al. (2021).

1839 Composition. Under the primary reference phylogeny, Pachyrostra comprises Achelousaurus

1840 horneri and Pachyrhinosaurus spp.

1841 Synonyms. No other taxon names are currently in use for the same or approximate clade.

1842 Comments. The name was first (informally) defined by Fiorillo \& Tykoski (2012) who applied

1843 the minimum-clade definition and used Achelousaurus horneri and Pachyrhinosaurus

1844 canadensis as the internal specifiers. We formalize this definition.

Peer) reviewing PDF | (2021:04:60693:1:1:NEW 8 Sep 2021) 
1845

1846 Panoplosaurini [new clade name]

1847 Registration number: 644

1848 Definition. The largest clade containing Panoplosaurus mirus Lambe, 1919 but not Nodosaurus

1849 textilis Marsh, 1889 and Struthiosaurus austriacus Bunzel, 1871. This is a maximum-clade

1850 definition. Abbreviated definition: $\max \nabla$ (Panoplosaurus mirus Lambe, $1919 \sim$ Nodosaurus

1851 textilis Marsh, 1889 \& Struthiosaurus austriacus Bunzel, 1871).

1852 Etymology. Derived from the stem of Panoplosaurus Lambe, 1919, the name of an included 1853 taxon, which combines the Greek words pan (all), hoplon (type of shield), and sauros (lizard, 1854 reptile).

1855 Reference phylogeny. Figure 5 of Rivera-Sylva et al. (2018a) is treated here as the primary 1856 reference phylogeny. Additional reference phylogenies include Figure 1 of Arbour et al. (2016), 1857 Figure 3 of Brown et al. (2017), and Figure 9 of Zheng et al. (2018).

1858 Composition. Under the primary reference phylogeny, Panoplosaurini comprises Animantarx 1859 ramaljonesi, 'Denversaurus' schlessmani, Edmontonia longiceps, Edmontonia rugosidens, 1860 Panoplosaurus mirus, Texasetes pleurohalio, and the Argentinian ankylosaur.

1861 Synonyms. The name Panoplosaurinae Nopcsa, 1929 has been recently suggested for the same 1862 clade (e.g., Rivera-Sylva et al., 2018a; see also 'Comments’ below). Additionally, Bakker (1988) 1863 coined the name Edmontoniinae for Edmontonia rugosidens, Edmontonia longiceps, and 1864 Denversaurus schlessmani and Edmontoniidae to include Edmontoniinae and Panoplosaurinae; 1865 no phylogenetic definition was proposed for either and neither clade name has been widely used 1866 since.

1867 Comments. The grouping, here covered under the name Panoplosaurini, has previously been 1868 suggested to be named Panoplosaurinae (Rivera-Sylva et al., 2018a). No (informal) 1869 phylogenetic definition for Panoplosaurinae has ever been published in the peer-reviewed 1870 literature, though Burns (2015) proposed "all Late Cretaceous nodosaurids more closely related 1871 to Panoplosaurus than to Pawpawsaurus" in his dissertation, and the name itself has not been 1872 widely used. Bakker (1988) provided a diagnosis of Panoplosaurinae, as nodosaurids with 1873 lumpy armor and expanded internarial bridges, which contained the two species of 1874 Panoplosaurus he recognized (Panoplosaurus mirus and Panoplosaurus sp. 1, represented by 1875 ROM 1215). Alpha taxonomic reviews of the Campanian-Maastrichtian North American 
1876 nodosaurids generally recognize Panoplosaurus mirus, Edmontonia rugosidens, and Edmontonia 1877 longiceps as valid taxa (e.g., Carpenter, 2001) and these typically form a clade (e.g. Kirkland, 1878 1998, Vickaryous et al., 2004; Thompson et al., 2012; Yang et al. 2013), sometimes with 1879 additional taxa such as Texasetes (Arbour et al., 2016; Rivera-Sylva et al., 2018a) or Animantarx 1880 (Hill et al., 2003). Rivera-Sylva et al. (2018a) noted that the grouping Animantarx,

1881 'Denversaurus', Edmontonia, Panoplosaurus, Texasetes, and an unnamed Argentinian 1882 ankylosaur could bear the name Panoplosaurinae. In several recent analyses Edmontonia and 1883 Panoplosaurus are found as the sister clade to a clade containing Struthiosaurus (Arbour et al., 1884 2016; Brown et al., 2017; Rivera-Sylva et al., 2018a), here named Struthiosaurini (see the name 1885 entry). Owing to the fact that the 'Panoplosaurus clade' is nested within Nodosaurinae, we 1886 prefer to use a name that implies a lesser inclusiveness. The suffix -inae (as in Panoplosaurinae) 1887 is typically associated with the rank of 'subfamily' under the $I C Z N$. Therefore, the use of the 1888 suffix '-inae' for the 'Panoplosaurus clade', without discussing the phylogenetic context, may 1889 suggest that Panoplosaurinae represents a clade outside Nodosaurinae. When the widely used 1890 suffix -ini (typically associated with the rank of 'tribe') is applied, such confusion is eliminated. 1891

\section{Parasaurolophini Glut, 1997 [converted clade name]} Registration number: 645

1894 Definition. The largest clade containing Parasaurolophus walkeri Parks, 1922 but not 1895 Aralosaurus tuberiferus Rozhdestvensky, 1968, Lambeosaurus lambei Parks, 1923 and 1896 Tsintaosaurus spinorhinus Young, 1958. This is a maximum-clade definition. Abbreviated definition: $\max \nabla$ (Parasaurolophus walkeri Parks, $1922 \sim$ Aralosaurus tuberiferus

1898 Rozhdestvensky, 1968 \& Lambeosaurus lambei Parks, 1923 \& Tsintaosaurus spinorhinus 1899 Young, 1958).

1900 Reference phylogeny. Figure 18 of Prieto-Márquez et al. (2020) is treated here as the primary 1901 reference phylogeny. Additional reference phylogenies include Figure 5 of Kobayashi et al. 1902 (2019), Figure 11 of Prieto-Márquez et al. (2019), Figure 9 of Zhang et al. (2019), Figure 5 of 1903 Zhang et al. (2020), Figure 7 of Kobayashi et al. (2021), and Figure 10 of Longrich et al. (2021).

1904 Composition. Under the primary reference phylogeny, Parasaurolophini comprises 1905 Charonosaurus jiayinensis and Parasaurolophus spp.

1906 Synonyms. No other taxon names are currently in use for the same or approximate clade. 
1907 Comments. The name was first (informally) defined by Prieto-Márquez et al. (2013) who

1908

1909

1910

1911

1912

1913

1914

1915

1916

1917

1918

1919

1920

1921

1922

1923

1924

1925

1926

1927

1928

1929

1930

1931

1932

1933

1934

1935

1936

1937 applied the maximum-clade definition and used Parasaurolophus walkeri as the internal specifier and Lambeosaurus lambei, Tsintaosaurus spinorhinus, and Aralosaurus tuberiferus as the external specifiers. We formalize this definition.

\section{Polacanthinae Lapparent \& Lavocat, 1955 [converted clade name]}

\section{Registration number: 646}

Definition. The largest clade within Ankylosauridae or Nodosauridae containing Polacanthus foxii Owen in Anonymous, 1865 but not Ankylosaurus magniventris Brown, 1908 and

Nodosaurus textilis Marsh, 1889. This is a maximum-clade definition. Abbreviated definition: $\max \nabla \in$ Ankylosauridae $\vee$ Nodosauridae (Polacanthus foxii Owen in Anonymous, 1865 Ankylosaurus magniventris Brown, 1908 \& Nodosaurus textilis Marsh, 1889).

Reference phylogeny. Figure 9 of Yang et al. (2013) is treated here as the primary reference phylogeny. Additional reference phylogenies include Figure 3 of Kirkland (1998), Figure 2 of Thompson et al. (2012), Figure 1 of Arbour et al. (2016), Figure 5 of Rivera-Sylva et al. (2018a), and Figure 9 of Zheng et al. (2018).

Composition. Under the primary reference phylogeny, Polacanthinae comprises Polacanthus foxii and Taohelong jinchengensis.

Synonyms. Jaekel (1910) introduced the name Polacanthidae to include ankylosaurs that appeared intermediate between Ankylosauridae and Nodosauridae. Kirkland (1998) were the first to assess 'polacanthids' using cladistic methods and found them to be a clade of earlydiverging ankylosaurids, and as such should preferably be called Polacanthinae rather than Polacanthidae, to eliminate the possible confusion that Ankylosauridae and Polacanthidae refer to mutually exclusive clades. Carpenter (2001) argued that Polacanthidae was instead valid and defined the name as all ankylosaurs closer to Gastonia than to Edmontonia or Euoplocephalus. Comments. The name Polacanthinae was (informally) defined before by Yang et al. (2013), who used Polacanthus foxii as the internal specifier and Ankylosaurus magniventris and Panoplosaurus mirus as the external specifiers. Kirkland (1998) diagnosed Polacanthinae as comprising ankylosaurs with an ankylosaurid-like skulls, nearly straight and parallel tooth rows, long basipterygoid processes, well-developed acromion arising from dorsal margin of scapula, ventrally flexed ischia, coossified pelvic osteoderms forming pelvic shield, pectoral osteoderms 
1938 elongate spikes with posterior grooves, and caudal osteoderms large, elongate laterally directed, 1939 and with hollow bases. Kirkland (1998) initially found Polacanthinae at the base of 1940 Ankylosauridae including Gastonia, Polacanthus, and Mymoorapelta and also referred 1941 Hoplitosaurus and Hylaeosaurus to the clade. Arbour et al. (2016), Rivera-Sylva et al. (2018a), 1942 and Zheng et al. (2018) inferred what could be called Polacanthinae at the base of 1943 Nodosauridae, including Polacanthus foxii and Hoplitosaurus marshi. Polacanthinae is poorly 1944 supported in most phylogenetic analyses yet frequently referenced in the literature. Taxa 1945 typically referred to as 'polacanthines' most often form a grade of early-diverging nodosaurids 1946 (e.g., Thompson et al., 2012; Brown et al., 2017). Additional taxonomic and phylogenetic 1947 revisions are needed to provide an assessment of Polacanthinae. We define the name here to 1948 ensure that it is applicable either within Ankylosauridae or Nodosauridae. If the 'Polacanthus 1949 clade' is reconstructed outside the Ankylosauridae + Nodosauridae node, the name 1950 Polacanthinae becomes inapplicable and the preferred name for the grouping should probably be 1951 Polacanthidae (not defined here).

1952

1953

Protoceratopsidae Granger \& Gregory, 1923 [converted clade name]

Registration number: 647

1955 Definition. The largest clade containing Protoceratops andrewsi Granger \& Gregory, 1923 but 1956 not Ceratops montanus Marsh, 1888, Leptoceratops gracilis Brown, 1914b, and Triceratops

1957 horridus Marsh, 1889. This is a maximum-clade definition. Abbreviated definition: $\max \nabla$ 1958 (Protoceratops andrewsi Granger \& Gregory, $1923 \sim$ Ceratops montanus Marsh, 1888 \& 1959 Leptoceratops gracilis Brown, 1914b \& Triceratops horridus Marsh, 1889).

1960 Reference phylogeny. Figure 10 of Morschhauser et al. (2019) is treated here as the primary 1961 reference phylogeny. Additional reference phylogenies include Figure S1 of Knapp et al. (2018),

1962 Figure 8A of Arbour et al. (2019), Figure 3 of Yu et al. (2020), and Figure 4 of Yu et al. (2020).

1963 Composition. Under the primary reference phylogeny, Protoceratopsidae comprises

1964 Bagaceratops rozhdestvenskyi, Magnirostris dodsoni (?= Bagaceratops rozhdestvenskyi; see 1965 Czepiński, 2020), and Protoceratops spp.

1966 Synonyms. No other taxon names are currently in use for the same or approximate clade.

1967 Comments. The name Protoceratopsidae has been (informally) defined before by Sereno (1998, 1968 2005) who applied a maximum-clade definition and used Protoceratops andrewsi as the internal 
1969 specifier and Triceratops horridus as the external specifier. We include two additional external 1970 specifiers Ceratops montanus and Leptoceratops gracilis. C. montanus was added because the 1971 name Protoceratopsidae has been traditionally applied to the sister taxon of Ceratopsoidea, and

1972 L. gracilis was included to ensure that Protoceratopsidae and Leptoceratopsidae remain 1973 mutually exclusive clades.

1974

1975 Rhabdodontidae Weishampel et al., 2003 [converted clade name]

1976 Registration number: 648

1977 Definition. The smallest clade containing Rhabdodon priscus Matheron, 1869 and Zalmoxes 1978 robustus (Nopcsa, 1900). This is a minimum-clade definition. Abbreviated definition: $\min \nabla$ 1979 (Rhabdodon priscus Matheron, 1869 \& Zalmoxes robustus [Nopcsa, 1900]).

1980 Reference phylogeny. Figure 4 of Madzia et al. (2018) is treated here as the primary reference

1981 phylogeny. Additional reference phylogenies include Figure 3 of Madzia et al. (2018), Figure 20 1982 of Verdú et al. (2018), Figure 25 of Herne et al. (2019), Figure 3 of Párraga \& Prieto-Márquez 1983 (2019), Figure 2 of Dieudonné et al. (2020), and Figure 9 of Verdú et al. (2020).

1984 Composition. Under the primary reference phylogeny, Rhabdodontidae comprises Rhabdodon 1985 priscus, Zalmoxes robustus, Zalmoxes shqiperorum, Mochlodon suessi, and Mochlodon vorosi.

1986 Synonyms. No other taxon names are currently in use for the same or approximate clade.

1987 Comments. The name Rhabdodontidae was first (informally) defined by Weishampel et al.

1988 (2003: 69) who used the minimum-clade definition and selected Rhabdodon priscus and 1989 Zalmoxes robustus as the internal specifiers. Sereno (2005) later used a maximum-clade 1990 definition, using Rhabdodon priscus as the internal specifier and Parasaurolophus walkeri as the 1991 external specifier. We formalize the former, minimum-clade, definition. A definition similar in 1992 effect to that of Sereno (2005) is applied to Rhabdodontomorpha.

1993

1994 Rhabdodontomorpha Dieudonné et al., 2016 [converted clade name]

1995 Registration number: 649

1996 Definition. The largest clade containing Rhabdodon priscus Matheron, 1869 but not

1997 Hypsilophodon foxii Huxley, 1869 and Iguanodon bernissartensis Boulenger in Beneden, 1881.

1998 This is a maximum-clade definition. Abbreviated definition: max $\nabla$ (Rhabdodon priscus

Peer] reviewing PDF | (2021:04:60693:1:1:NEW 8 Sep 2021) 
1999 Matheron, 1869 Hypsilophodon foxii Huxley, 1869 \& Iguanodon bernissartensis Boulenger in 2000 Beneden, 1881).

2001 Reference phylogeny. Figure 2 of Dieudonné et al. (2020) is treated here as the primary 2002 reference phylogeny. Additional reference phylogenies include Figure 4 of Madzia et al. (2018), 2003 Figure 25 of Herne et al. (2019), and Figure 57 of Barta \& Norell (2021).

2004 Composition. Under the primary reference phylogeny, Rhabdodontomorpha comprises

2005 Muttaburrasaurus langdoni, Fostoria dhimbangunmal, the 'Vegagete ornithopod', and members 2006 of the clade Rhabdodontidae.

2007 Synonyms. No other taxon names are currently in use for the same or approximate clade.

2008 Comments. The application of Rhabdodontomorpha has been described, and (informally) 2009 proposed definitions have been discussed, by Madzia et al. (2018: Appendix 1) and Madzia et al. 2010 (2020: Table 1). We therefore refer to these studies for details. Our formalized maximum-clade 2011 definition is similar to that of Madzia et al. (2020) in that it uses Rhabdodon priscus as the 2012 internal specifier and Iguanodon bernissartensis as the external specifier. We have further added 2013 a second external specifier, Hypsilophodon foxii, to prevent its inclusion to Rhabdodontomorpha 2014 under phylogenies similar to that of Norman (2015: Fig. 50).

2015

2016 Saphornithischia [new clade name]

2017 Registration number: 747

2018 Definition. The smallest clade containing Heterodontosaurus tucki Crompton \& Charig, 1962, 2019 Iguanodon bernissartensis Boulenger in Beneden, 1881, Stegosaurus stenops Marsh, 1887, and 2020 Triceratops horridus Marsh, 1889. This is a minimum-clade definition. Abbreviated definition: $2021 \min \nabla$ (Heterodontosaurus tucki Crompton \& Charig, 1962 \& Iguanodon bernissartensis 2022 Boulenger in Beneden, 1881 \& Stegosaurus stenops Marsh, 1887 \& Triceratops horridus Marsh, 2023 1889).

2024 Etymology. Derived from the Greek safis (clear, definite) and formed to include all members of 2025 Ornithischia whose placement within the clade is well established.

2026 Reference Phylogeny. Figure 4 of Madzia et al. (2018) is treated here as the primary reference 2027 phylogeny. Additional reference phylogenies include Figure 25 of Herne et al. (2019), Yang et 2028 al. (2020), and Figure 57 of Barta \& Norell (2021). 
2029 Composition. Under the primary reference phylogeny, Saphornithischia comprises

2030 Pisanosaurus mertii and members of the clades Heterodontosauridae and Genasauria.

2031 Synonyms. Under alternative topologies, where Heterodontosauridae is reconstructed within

2032 Neornithischia (e.g., Butler, 2005; Xu et al., 2006; Dieudonné et al., 2020), Saphornithischia

2033 would be a heterodefinitional synonym of Genasauria.

2034 Comments. Given the repeated inference of heterodontosaurids outside Genasauria in multiple

2035 studies (e.g., Butler et al., 2008; Boyd, 2015; Han et al., 2018; Madzia et al., 2018; Andrzejewski

2036 et al., 2019; Herne et al., 2019; Yang et al., 2020) and the uncertainty surrounding the potential

2037 ornithischian affinities of Pisanosaurus mertii (Agnolín \& Rozadilla, 2018, Baron, 2019; Desojo

2038 et al., 2020) and members of the Silesauridae (Langer \& Ferigolo, 2013; Cabreira et al., 2016;

2039 Pacheco et al., 2019; Müller \& Garcia, 2020), we provide a new clade name to cover taxa whose

2040 placement within Ornithischia is well-supported. Note that the internal specifiers Stegosaurus

2041 stenops and Triceratops horridus are not included in the primary reference phylogeny. The

2042 former belongs to Thyreophora (e.g., Maidment et al., 2020), while the latter is part of

2043 Marginocephalia (see, e.g., Morschhauser et al., 2019; Fowler \& Freedman Fowler, 2020). Both

2044 these clades are indicated on Figure 4 of Madzia et al. (2018).

2045

2046 Saurolophinae Brown, 1914a [converted clade name]

2047 Registration number: 650

2048 Definition. The largest clade containing Saurolophus osborni Brown, 1912 but not

2049 Lambeosaurus lambei Parks, 1923, provided that it does not include Hadrosaurus foulkii Leidy, 2050 1858. This is a maximum-clade definition. Abbreviated definition: $\max \nabla$ (Saurolophus osborni 2051 Brown, $1912 \sim$ Lambeosaurus lambei Parks, 1923 | Hadrosaurus foulkii Leidy, 1858).

2052 Reference phylogeny. Figure 18 of Prieto-Márquez et al. (2020) is treated here as the primary 2053 reference phylogeny. Additional reference phylogenies include Figure 5 of Kobayashi et al. 2054 (2019), Figure 11 of Prieto-Márquez et al. (2019), Figure 9 of Zhang et al. (2019), Figure 5 of 2055 Zhang et al. (2020), Figure 7 of Kobayashi et al. (2021), and Figure 10 of Longrich et al. (2021). 2056 Composition. Under the primary reference phylogeny, Saurolophinae comprises ?Gryposaurus

2057 alsatei, Naashoibitosaurus ostromi, members of the clades Brachylophosaurini, Edmontosaurini, 2058 Kritosaurini, and Saurolophini, and the specimen 'PASAC 1 ('Sabinosaur')'. 
2059 Synonyms. Following the widespread application of the Principle of Coordination, under which 2060 Hadrosaurinae has to be attributed to Cope (1869), the name Hadrosaurinae is generally 2061 considered to have priority over Saurolophinae, even though the latter was coined four years 2062 earlier (Saurolophinae Brown, 1914a; Hadrosaurinae Lambe, 1918). In recent years, both 2063 Hadrosaurinae and Saurolophinae, have been used for the sister taxon of Lambeosaurinae. The 2064 selection of the proper name has traditionally depended on whether the clade includes 2065 Hadrosaurus foulkii or not (Fig. 5). In the cases in which H. foulkii falls within the smallest 2066 clade containing Saurolophus osborni and Lambeosaurus lambei, and within the 'Saurolophus 2067 branch', the name Hadrosaurinae is preferred (e.g., Cruzado-Caballero \& Powell, 2017; Xing et 2068 al., 2017; Kobayashi et al., 2019; Zhang et al., 2020). However, when H. foulkii falls outside the 2069 clade, the name Saurolophinae is used (e.g., Prieto-Márquez et al., 2019; Prieto-Márquez et al., 2070 2020; Gates et al., 2021; Kobayashi et al., 2021; McDonald et al., 2021; Ramírez-Velasco et al., 2071 2021).

2072 Comments. The name Saurolophinae has been (informally) defined before by Prieto-Márquez 2073 (2010) who applied a maximum-clade definition and used Saurolophus osborni as the internal 2074 specifier and Lambeosaurus lambei and Hadrosaurus foulkii as the external specifiers. Here, we

2075 formalize a maximum-clade definition of Saurolophinae that applies the name to the sister clade 2076 of Lambeosaurinae only on the condition that it does not contain H. foulkii. In turn, the name 2077 Hadrosaurinae is defined to be used for the 'Saurolophus branch' when H. foulkii falls within 2078 the clade. Although our definition may be considered similar to that of Prieto-Márquez (2010) it 2079 differs substantially because under our definition, the name Saurolophinae may become 2080 inapplicable.

2081

2082 Saurolophini Glut, 1997 [converted clade name]

2083 Registration number: 651

2084 Definition. The largest clade containing Saurolophus osborni Brown, 1912 but not

2085 Brachylophosaurus canadensis Sternberg, 1953, Edmontosaurus regalis Lambe, 1917, 2086 Hadrosaurus foulkii Leidy, 1858, and Kritosaurus navajovius Brown, 1910. This is a maximum2087 clade definition. Abbreviated definition: $\max \nabla$ (Saurolophus osborni Brown, 1912 2088 Brachylophosaurus canadensis Sternberg, 1953 \& Edmontosaurus regalis Lambe, 1917 \& 2089 Hadrosaurus foulkii Leidy, 1858 \& Kritosaurus navajovius Brown, 1910). 
2090 Reference phylogeny. Figure 18 of Prieto-Márquez et al. (2020) is treated here as the primary 2091 reference phylogeny. Additional reference phylogenies include Figure 5 of Kobayashi et al. 2092 (2019), Figure 11 of Prieto-Márquez et al. (2019), Figure 9 of Zhang et al. (2019), Figure 5 of 2093 Zhang et al. (2020), Figure 7 of Kobayashi et al. (2021), and Figure 10 of Longrich et al. (2021). 2094 Composition. Under the primary reference phylogeny, Saurolophini comprises Augustynolophus 2095 morrisi, Prosaurolophus maximus, and Saurolophus spp.

2096 Synonyms. No other taxon names are currently in use for the same or approximate clade.

2097 Comments. The name Saurolophini has been (informally) defined before (Sereno, 2005; Prieto-

2098 Márquez et al., 2014). Both these definitions were maximum-clade and used Saurolophus 2099 osborni as the internal specifier and Edmontosaurus regalis and Maiasaura peeblesorum 2100 (Sereno, 2005) or Brachylophosaurus canadensis, Edmontosaurus regalis, Kritosaurus 2101 navajovius, and Lambeosaurus lambei (Prieto-Márquez et al., 2014) as the external specifiers. 2102 Here we apply a definition similar to that of Prieto-Márquez et al. (2014) but remove L. lambei 2103 and instead add Hadrosaurus foulkii.

2104

\section{Shamosaurinae Tumanova, 1983 [converted clade name]}

Registration number: 652

Definition. The largest clade containing Gobisaurus domoculus Vickaryous et al., 2001 and Shamosaurus scutatus Tumanova, 1983 but not Ankylosaurus magniventris Brown, 1908. This is a maximum-clade definition. Abbreviated definition: $\max \nabla$ (Gobisaurus domoculus Vickaryous et al., 2001 \& Shamosaurus scutatus Tumanova, 1983 Ankylosaurus magniventris Brown,

2112 Reference phylogeny. Figure 11 of Arbour \& Currie (2016) is treated here as the primary 2113 reference phylogeny. Additional reference phylogenies include Figure 1 of Arbour et al. (2016), 2114 Figure 8 of Arbour \& Evans (2017), and Figure 5 of Rivera-Sylva et al. (2018a).

2115 Composition. Under the primary reference phylogeny, Shamosaurinae comprises Gobisaurus 2116 domoculus and Shamosaurus scutatus.

2117 Synonyms. No other taxon names are currently in use for the same or approximate clade.

2118 Comments. Tumanova (1987) described Shamosaurinae based on a list of diagnostic features; 2119 shamosaurines were ankylosaurids with narrow anterior snouts, angle of the orbital plane with 2120 the skull axis less than $25^{\circ}$, anterior wall of the pterygois inclined posteriorly, occipital condyle a 
2121 wide oval, pterygoids fused with the basisphenoid, small interpterygoid fenestra, and orbits at the

2122 midlength of the skull. Shamosaurinae is not reconstructed in all recent phylogenetic analyses, as

2123 Shamosaurus and Gobisaurus are sometimes inferred as successive outgroups to Ankylosaurinae

2124 rather than as a clade (e.g. Thompson et al., 2012; Wiersma \& Irmis, 2018). We provide a

2125 maximum-clade definition that makes Shamosaurinae applicable only under the topologies in

2126 which Shamosaurus and Gobisaurus are closely related to each other than either is to

2127 Ankylosaurus.

2128

2129 Stegosauria Marsh, 1877b [converted clade name]

2130 Registration number: 653

2131 Definition. The largest clade containing Stegosaurus stenops Marsh, 1887 but not Ankylosaurus

2132 magniventris Brown, 1908. This is a maximum-clade definition. Abbreviated definition: max $\nabla$

2133 (Stegosaurus stenops Marsh, 1887 Ankylosaurus magniventris Brown, 1908).

2134 Reference phylogeny. Figure 12 of Maidment et al. (2020) is treated here as the primary

2135 reference phylogeny. Additional reference phylogenies include Figure 11 of Maidment et al.

2136 (2008), Figure 1 of Raven and Maidment (2017), and Figure 1 of Dieudonné et al. (2020).

2137 Composition. Under the primary reference phylogeny, Stegosauria comprises Isaberrysaura

2138 mollensis, Gigantspinosaurus sichuanensis, and members of the clades Stegosauridae and

2139 Huayangosauridae.

2140 Synonyms. No other taxon names are currently in use for the same or approximate clade.

2141 Comments. The name Stegosauria has been (informally) defined before (Galton, 1997; Sereno,

2142 1998; Galton and Upchurch, 2004; Sereno, 2005) using Stegosaurus (Galton, 1997; Sereno,

2143 1998; Galton and Upchurch, 2004) or Stegosaurus stenops (Sereno, 2005) as the internal

2144 specifier and Ankylosaurus (Galton, 1997; Sereno, 1998), Ankylosauria (Galton and Upchurch,

2145 2004), or Ankylosaurus magniventris (Sereno, 2005) as the external specifiers. Since Stegosauria

2146 has never been proposed an alternative use, we use $S$. stenops as the internal specifier and $A$.

2147 magniventris as the external specifier. Note that A. magniventris is not included in the primary

2148 reference phylogeny. See Figure 3 of Thompson et al. (2012) for its placement with respect to

2149 Stegosauria.

2150

2151 Stegosauridae Marsh, 1880 [converted clade name]

Peer] reviewing PDF | (2021:04:60693:1:1:NEW 8 Sep 2021) 
2152 Registration number: 654

2153 Definition. The largest clade containing Stegosaurus stenops Marsh, 1887 but not

2154 Huayangosaurus taibaii Dong et al., 1982. This is a maximum-clade definition. Abbreviated

2155 definition: $\max \nabla$ (Stegosaurus stenops Marsh, 1887 Huayangosaurus taibaii Dong et al., 2156 1982).

2157 Reference phylogeny. Figure 12 of Maidment et al. (2020) is treated here as the primary

2158 reference phylogeny. Additional reference phylogenies include Figure 11 of Maidment et al.

2159 (2008) and Figure 1 of Raven and Maidment (2017).

2160 Composition. Under the primary reference phylogeny, Stegosauridae comprises Adratiklit

2161 boulahfa, Alcovasaurus longispinus, Dacentrurus armatus, Hesperosaurus mjosi,

2162 Jiangjunosaurus junggarensis, Kentrosaurus aethiopicus, Loricatosaurus priscus, Miragaia

2163 longicollum, Paranthodon africanus, Stegosaurus homheni, Stegosaurus stenops, and

2164 Tuojiangosaurus multispinus.

2165 Synonyms. No other taxon names are currently in use for the same or approximate clade.

2166 Comments. The name Stegosauridae was first (informally) defined by Sereno (1998, 2005) who

2167 used the maximum-clade definition and selected Stegosaurus stenops as the internal specifier and

2168 Huayangosaurus taibaii as the external specifier. We formalize this definition.

2169

2170 Struthiosaurini [new clade name]

2171 Registration number: 655

2172 Definition. The largest clade containing Struthiosaurus austriacus Bunzel, 1871 but not

2173 Nodosaurus textilis Marsh, 1889 and Panoplosaurus mirus Lambe, 1919. This is a maximum-

2174 clade definition. Abbreviated definition: $\max \nabla$ (Struthiosaurus austriacus Bunzel, 1871

2175 Nodosaurus textilis Marsh, 1889 \& Panoplosaurus mirus Lambe, 1919).

2176 Etymology. Derived from the stem of Struthiosaurus Bunzel, 1871, the name of an included

2177 taxon, which combines the Latin word struthio (ostrich) and Greek sauros (lizard, reptile).

2178 Reference phylogeny. Figure 5 of Rivera-Sylva et al. (2018a) is treated here as the primary

2179 reference phylogeny. Additional reference phylogenies include Figure 1 of Arbour et al. (2016),

2180 Figure 3 of Brown et al. (2017), and Figure 9 of Zheng et al. (2018). 
2181 Composition. Under the primary reference phylogeny, Struthiosaurini comprises Europelta

2182 carbonensis, Hungarosaurus tormai, Pawpawsaurus campbelli, Stegopelta landerensis, and

2183 Struthiosaurus spp.

2184 Synonyms. The name Struthiosaurinae Nopcsa, 1923 has been recently used for an approximate 2185 clade (Kirkland et al., 2013; Blows \& Honeysett, 2014; Villanueva-Amadoz et al., 2015). No

2186 other taxon names are currently in use for the same or approximate clade.

2187 Comments. A grouping similar to that covered here under the name Struthiosaurini has 2188 previously been named Struthiosaurinae (Kirkland et al., 2013). The name Struthiosaurinae was 2189 (informally) defined by Kirkland et al. (2013) who applied the maximum-clade definition and 2190 used Europelta as the internal specifier and Cedarpelta, Peloroplites, Sauropelta, and 2191 Edmontonia as the external specifiers. Struthiosaurinae was considered to represent the clade of 2192 Late Cretaceous European nodosaurids. However, Kirkland et al. (2013) did not include a 2193 character matrix or phylogenetic analysis in their study and have not yet published a follow-up 2194 paper with results indicating the extent of their Struthiosaurinae. They provided, however, a list 2195 of diagnostic characters. According to Kirkland et al. (2013), Struthiosaurinae includes 2196 nodosaurid ankylosaurs with narrow predentaries, nearly horizontal and unfused quadrates, 2197 quadrate condyls that are 3 times transversely wider than long, premaxillary teeth and dentary 2198 teeth that are near the predentary symphysis, dorsally arched sacra, an acromion process dorsal to 2199 the midpoint of the scapulocoracoid suture, straight ischia with a straight dorsal margin, long 2200 slender limbs, a sacral shield, and erect sacral osteoderms with flat bases. This suite of characters 2201 was considered to unite Anoplosaurus, Europelta, Hungarosaurus, and Struthiosaurus, but many 2202 of these characters have a broad distribution in Ankylosauria and Nodosauridae (Ösi, 2015). 2203 Arbour et al. (2016) reconstructed a clade containing Ahshislepelta, Europelta, Hungarosaurus, 2204 Niobrarasaurus, Nodosaurus, Pawpawsaurus, Stegopelta, Struthiosaurus, and the 'Paw Paw 2205 juvenile' as the sister clade to that containing Edmontonia, which would thus be considered 2206 Struthiosaurinae. Brown et al. (2017) added Borealopelta to Arbour et al. (2016) and 2207 reconstructed a clade of Borealopelta, Europelta, Hungarosaurus, and Pawpawsaurus; 2208 Stegopelta and Struthiosaurus were outside of this clade and sister to Edmontonia, 2209 'Denversaurus', and Panoplosaurus. As was the case with Panoplosaurinae, owing to the fact 2210 that the 'Struthiosaurus clade' is nested within Nodosaurinae, we prefer to use a name that 2211 implies a lesser inclusiveness (that is, -ini rather than-inae). The use of Struthiosaurinae, 
2212 without discussing the phylogenetic context, may suggest that Struthiosaurinae and

2213 Nodosaurinae are mutually exclusive clades. When the suffix -ini is applied, such confusion is 2214 eliminated. Note that the recent use of Struthiosaurinae has been largely limited to mentions of

2215 Kirkland et al.’s (2013) application of the name (Blows \& Honeysett, 2014; Villanueva-Amadoz 2216 et al., 2015).

\section{Styracosterna Sereno, 1986 [converted clade name]}

2219 Registration number: 656

Definition. The largest clade containing Iguanodon bernissartensis Boulenger in Beneden, 1881 but not Camptosaurus dispar (Marsh, 1879). This is a maximum-clade definition. Abbreviated definition: max $\nabla$ (Iguanodon bernissartensis Boulenger in Beneden, $1881 \sim$ Camptosaurus dispar [Marsh, 1879]).

Reference phylogeny. Figure 12 of Madzia et al. (2020) is treated here as the primary reference phylogeny. Additional reference phylogenies include Figure 20 of Verdú et al. (2018), Figure 11 of McDonald et al. (2021), Figure 11 of Santos-Cubedo et al. (2021), and Figure 9 of Verdú et al. 2227 (2020).

Composition. Under the primary reference phylogeny, Styracosterna comprises Cedrorestes crichtoni, Cumnoria prestwichii, Dakotadon lakotaensis, Draconyx loureioi, Fukuisaurus tetoriensis, Hippodraco scutodens, Iguanacolossus fortis, Lanzhousaurus magnidens, Muttaburrasaurus langdoni, Osmakasaurus depressus, Owenodon hoggii, Planicoxa venenica,

2232 Theiophytalia kerri, Uteodon aphanoecetes, Yunganglong datongensis, and members of the 2233 clade Hadrosauriformes.

2234 Synonyms. No other taxon names are currently in use for the same or approximate clade. 2235 Comments. The name Styracosterna was first (informally) defined by Sereno (1998: 62) who used the maximum-clade definition and selected Parasaurolophus as the internal specifier and Camptosaurus as the external specifier. We prefer to use Iguanodon bernissartensis as the external specifier to maintain the 'node-branch triplet' ('node-stem triplet' of Sereno [1998: 5254]) comprising Ankylopollexia, Camptosauridae, and Styracosterna (all formally defined in the present paper). The inclusion of a different external specifier does not change the extent of Styracosterna under any of the published phylogeny inferences. 


\section{Thescelosauridae Sternberg, 1937 [converted clade name]}

\section{Registration number: 657}

2245 Definition. The largest clade containing Thescelosaurus neglectus Gilmore, 1913 but not

2246 Iguanodon bernissartensis Boulenger in Beneden, 1881, provided that it does not include

2247 Hypsilophodon foxii Huxley, 1869. This is a maximum-clade definition. Abbreviated definition:

$2248 \max \nabla$ (Thescelosaurus neglectus Gilmore, $1913 \sim$ Iguanodon bernissartensis Boulenger in

2249 Beneden, $1881 \mid \sim$ Hypsilophodon foxii Huxley, 1869).

2250 Reference phylogeny. Figure 4 of Madzia et al. (2018) is treated here as the primary reference

2251 phylogeny. Additional reference phylogenies include Figure 25 of Herne et al. (2019) and Figure

225257 of Barta \& Norell (2021).

2253 Composition. Under the primary reference phylogeny, Thescelosauridae comprises members of 2254 the clades Thescelosaurinae and Orodrominae.

2255 Synonyms. The name Parksosauridae Buchholz, 2002 has been used recently for the same 2256 contents (Boyd, 2015; Rivera-Sylva et al., 2018b). No other taxon names are currently in use for 2257 the same or approximate clade.

2258 Comments. The name Thescelosauridae has been (informally) defined before (Brown et al., 2259 2013; Madzia et al., 2018). Both these definitions were minimum-clade and used Thescelosaurus 2260 neglectus and Orodromeus makelai as the internal specifiers. Madzia et al. (2018) further added 2261 one external specifier, Iguanodon bernissartensis, to ensure that the name is applicable with a 2262 similar circumscription (see Madzia et al., 2018: Appendix 1 for details). We apply a complex 2263 maximum-clade definition to ensure that Thescelosauridae is not inferred within 2264 Hypsilophodontidae; for example under the potential topology in which Hypsilophodon is the 2265 sister taxon to a Thescelosaurinae + Orodrominae node. Even though no such phylogenetic 2266 hypothesis has been proposed, the placement of taxa 'traditionally' dubbed the 'hypsilophodonts' 2267 is highly pliable across studies (Han et al., 2018; Madzia et al., 2018; Andrzejewski et al., 2019; 2268 Herne et al., 2019; Dieudonné et al., 2020; Rotatori et al., 2020; Yang et al., 2020) and often 2269 differ significantly even under different tree-search methods of a single dataset. Therefore, it can 2270 be expected that phylogeny inferences of the rootward neornithischian-ornithopod transitional 2271 segment of the ornithischian phylogenetic trees may result in such topology at some point. A 2272 maximum-clade definition with a single internal specifier (T. neglectus) was preferred to allow 2273 Thescelosauridae in use regardless of the relationship of T. neglectus to O. makelai. 
2275 Thescelosaurinae Sternberg, 1940 [converted clade name]

2276 Registration number: 658

2277 Definition. The largest clade within Hypsilophodontidae or Thescelosauridae containing

2278 Thescelosaurus neglectus Gilmore, 1913 but not Hypsilophodon foxii Huxley, 1869 and

2279 Orodromeus makelai Horner \& Weishampel, 1988. This is a maximum-clade definition.

2280 Abbreviated definition: $\max \nabla \in$ Hypsilophodontidae $\vee$ Thescelosauridae (Thescelosaurus

2281 neglectus Gilmore, 1913 Hypsilophodon foxii Huxley, 1869 \& Orodromeus makelai Horner \&

2282 Weishampel, 1988).

2283 Reference phylogeny. Figure 4 of Madzia et al. (2018) is treated here as the primary reference

2284 phylogeny. Additional reference phylogenies include Figure 25 of Herne et al. (2019) and Figure

228557 of Barta \& Norell (2021).

2286 Composition. Under the primary reference phylogeny, Thescelosaurinae comprises

2287 Notohypsilophodon comodorensis, Parksosaurus warreni, and Thescelosaurus spp.

2288 Synonyms. No other taxon names are currently in use for the same or approximate clade.

2289 Comments. The name Thescelosaurinae has been (informally) defined before (Brown and

2290 Druckenmiller, 2011; Boyd, 2015). Both these definitions were maximum-clade and used

2291 Thescelosaurus neglectus as the internal specifier and Orodromeus makelai and Hypsilophodon

2292 foxii (Brown and Druckenmiller, 2011) or Orodromeus makelai and Parasaurolophus walkeri

2293 (Boyd, 2015) as the external specifiers. Considering the 'traditional concept' of

2294 Thescelosaurinae, as a subclade of Thescelosauridae/'hypsilophodonts', and keeping in mind the

2295 unstable phylogenetic position of H. foxii (e.g., Madzia et al., 2018), we apply Thescelosaurinae

2296 only when it is inferred either within Thescelosauridae or Hypsilophodontidae (see Article 11.14

2297 of the $I C P N)$.

2298

2299 Thyreophora Nopcsa, 1915 [converted clade name]

2300 Registration number: 659

2301 Definition. The largest clade containing Ankylosaurus magniventris Brown, 1908 and

2302 Stegosaurus stenops Marsh, 1887 but not Iguanodon bernissartensis Boulenger in Beneden,

23031881 and Triceratops horridus Marsh, 1889. This is a maximum-clade definition. Abbreviated

Peer] reviewing PDF | (2021:04:60693:1:1:NEW 8 Sep 2021) 
2304 definition: $\max \nabla$ (Ankylosaurus magniventris Brown, 1908 \& Stegosaurus stenops Marsh, 1887

2305 Iguanodon bernissartensis Boulenger in Beneden, 1881 \& Triceratops horridus Marsh, 1889).

2306 Reference phylogeny. Figure 16 of Han et al. (2018) is treated here as the primary reference

2307 phylogeny. Additional reference phylogenies include Figure 4 of Madzia et al. (2018), Figure 25

2308 of Herne et al. (2019), Figure 1 of Dieudonné et al. (2020), Figure 12 of Yang et al. (2020), and

2309 Figure 57 of Barta \& Norell (2021).

2310 Composition. Under the primary reference phylogeny, Thyreophora comprises Scutellosaurus

2311 lawleri, Emausaurus ernsti, Scelidosaurus harrisonii, and members of the clade Eurypoda.

2312 Synonyms. No other taxon names are currently in use for the same or approximate clade.

2313 Comments. The name Thyreophora has been (informally) defined before (Sereno, 1998; Sereno, 2314 2005; Norman, 2021). All these definitions were maximum-clade. The definitions of Sereno

2315 (1998; 2005) used Ankylosaurus magniventris as the internal specifiers, and Triceratops horridus

2316 (Sereno, 1998) or T. horridus, Parasaurolophus walkeri, and Pachycephalosaurus wyomingensis

2317 (Sereno, 2005) as the external specifiers. In turn, Norman (2021) defined Thyreophora using

2318 Euoplocephalus and Stegosaurus as the internal specifiers and Hypsilophodon as the external

2319 specifier. In order to maintain the 'traditional' concept of Genasauria as a clade comprising

2320 Neornithischia and Thyreophora, the internal specifiers in the definition of Thyreophora are used

2321 from among the taxa representing the two major subclades - Ankylosauria (Ankylosaurus

2322 magniventris) and Stegosauria (Stegosaurus stenops) - and the external specifiers are used from

2323 among the taxa representing the neornithischian clades Ornithopoda (Iguanodon bernissartensis)

2324 and Marginocephalia (Triceratops horridus). Note that the internal specifier Ankylosaurus

2325 magniventris and the external specifier Triceratops horridus are not included in the primary

2326 reference phylogeny. The former belongs to Ankylosauria within Thyreophora (see, e.g.,

2327 Thompson et al., 2012), while the latter is part of Ceratopsia (e.g., Morschhauser et al., 2019).

2328

2329 Triceratopsini Longrich, 2011 [converted clade name]

2330 Registration number: 692

2331 Definition. The largest clade containing Triceratops horridus Marsh, 1889 but not

2332 Anchiceratops ornatus Brown, 1914c and Arrhinoceratops brachyops Parks, 1925. This is a

2333 maximum-clade definition. Abbreviated definition: max $\nabla$ (Triceratops horridus Marsh, 1889

2334 Anchiceratops ornatus Brown, 1914c \& Arrhinoceratops brachyops Parks, 1925).

Peer) reviewing PDF | (2021:04:60693:1:1:NEW 8 Sep 2021) 
2335 Reference phylogeny. Figure 9a of Fowler \& Freedman Fowler (2020) is treated here as the 2336 primary reference phylogeny. Additional reference phylogenies include Figure 11 of Longrich 2337 (2011), Figure 3 of Brown \& Henderson (2015), Figure 14 of Mallon et al. (2016), and Figure 3 2338 of Campbell et al. (2019).

2339 Composition. Under the primary reference phylogeny, Triceratopsini comprises Eotriceratops xerinsularis, Nedoceratops hatcheri, Ojoceratops fowleri, Torosaurus spp., and Triceratops spp.

2341 Synonyms. No other taxon names are currently in use for the same or approximate clade.

2342 Comments. The name was first (informally) defined by Longrich (2011) who applied the maximum-clade definition and used Triceratops horridus as the internal specifier and Anchiceratops ornatus and Arrhinoceratops brachyops as the external specifiers. We formalize this definition.

Tsintaosaurini Prieto-Márquez et al., 2013 [converted clade name]

Registration number: 660

Definition. The largest clade containing Pararhabdodon isonensis Casanovas Cladellas et al., 1993 and Tsintaosaurus spinorhinus Young, 1958 but not Aralosaurus tuberiferus

Rozhdestvensky, 1968, Lambeosaurus lambei Parks, 1923 and Parasaurolophus walkeri Parks, 1922. This is a maximum-clade definition. Abbreviated definition: $\max \nabla$ (Pararhabdodon isonensis Casanovas Cladellas et al., 1993 \& Tsintaosaurus spinorhinus Young, 1958 Aralosaurus tuberiferus Rozhdestvensky, 1968 \& Lambeosaurus lambei Parks, 1923 \& Parasaurolophus walkeri Parks, 1922).

Reference phylogeny. Figure 18 of Prieto-Márquez et al. (2020) is treated here as the primary reference phylogeny. Additional reference phylogenies include Figure 20 of Xing et al. (2017), Figure 5 of Kobayashi et al. (2019), Figure 11 of Prieto-Márquez et al. (2019), Figure 5 of Zhang et al. (2020), Figure 7 of Kobayashi et al. (2021), and Figure 11 of McDonald et al. (2021). Composition. Under the primary reference phylogeny, Tsintaosaurini comprises Pararhabdodon isonensis and Tsintaosaurus spinorhinus.

2362 Synonyms. No other taxon names are currently in use for the same or approximate clade.

2363 Comments. The name was first (informally) defined by Prieto-Márquez et al. (2013) who 2364 applied the minimum-clade definition and used Pararhabdodon isonensis and Tsintaosaurus 2365 spinorhinus as the internal specifiers. We preserve the original intent of Prieto-Márquez et al. 
2366 (2013) but prefer to use the maximum-clade definition. Pararhabdodon isonensis and

2367 Tsintaosaurus spinorhinus are used as the internal specifiers and representatives of Aralosaurini

2368 (Aralosaurus tuberiferus), Lambeosaurini (Lambeosaurus lambei), and Parasaurolophini

2369 (Parasaurolophus walkeri), as the external specifiers. The name Tsintaosaurini is inapplicable 2370 under some recent phylogenies (Prieto-Márquez et al., 2019; Gates et al., 2021; Longrich et al., 2371 2021).

2372

2373 Discussion

2374 Phylogeny reconstructions of some ornithischian clades currently face challenges that have an 2375 impact on the construction of the phylogenetic definitions of several taxon names. Below, we 2376 provide discussion of some topological conflicts.

\section{The phylogeny of early-diverging ornithischians}

The early evolution of Ornithischia and the phylogenetic relationships of taxa nested near the base of the clade are currently contentious, particularly with respect to the potential Triassic members of the clade. Ornithischians have been 'traditionally' represented by a single undisputed Triassic taxon, Pisanosaurus mertii Casamiquela, 1967. Recent reassessments of the type specimen of $P$. mertii showed, however, that the morphological features of the taxon are rather difficult to interpret and that it may represent either, a non-dinosaur dinosauriform from the clade Silesauridae (Agnolín \& Rozadilla, 2018, Baron, 2019) or an early-diverging ornithischian (Desojo et al., 2020). Even if $P$. mertii turns out to be a silesaurid, however, it may still represent an early-diverging ornithischian dinosaur as a few studies have proposed that silesaurids, a group of Anisian?Rhaetian (Middle and Late Triassic) dinosauriforms that are usually inferred to be the sister group to dinosaurs (e.g., Nesbitt et al., 2010; Peecook et al., 2013; Ezcurra, 2016; Cau, 2018; Ezcurra et al., 2020), may form an early clade of ornithischians (Langer \& Ferigolo, 2013; Cabreira et al., 2016; Pacheco et al., 2019) or a paraphyletic assemblage of early-diverging ornithischians (Müller \& Garcia, 2020). Such placement of the silesaurid taxa, especially the oldest known members referred to the group, would have considerable implications for the early evolution of dinosaurs as a whole because neither of the two other major dinosaur clades, theropods and sauropomodomorphs, are known from the Middle Triassic. 
2397 Pending additional studies, more focused on the basal dinosauriform-dinosaur transition, we do

2398 not define neither Silesauridae Langer et al., 2010 nor the recently proposed name

2399 Sulcimentisauria Martz \& Small, 2019. If formal definitions for the names are to be proposed in

2400 the future, the definitions should comply with all recently proposed phylogenies, including the

2401 possible paraphyletic 'dissolution’ of Silesauridae (Müller \& Garcia, 2020) that would make

2402 Sulcimentisauria, as (informally) defined by Martz \& Small (2019), applicable to a clade

2403 containing the vast majority of 'traditional' silesaurids and all 'core' ornithischians. One option

2404 is to restrict the use of Sulcimentisauria for a clade only when inferred within Silesauridae (e.g.,

2405 'max $\nabla \in$ Silesauridae (Silesaurus opolensis Dzik, $2003 \sim$ Asilisaurus kongwe Nesbitt et al.,

2406 2010)'), as originally intended by Martz \& Small (2019).

2407 Recently, Baron \& Barrett $(2017,2018)$ reconstructed the enigmatic dinosaur Chilesaurus

2408 diegosuarezi from the Tithonian (uppermost Jurassic) of Central Patagonian Cordillera in Chile

2409 to represent the earliest-diverging ornithischian, which was in striking contrast to the original

2410 inference of the taxon at the base of Tetanurae, within Theropoda (Novas et al., 2015). Despite

2411 that the proposed placement of Chilesaurus among early-diverging ornithischians does not have

2412 any impact on the use of particular clade names (except when the extent of Ornithischia is to be

2413 indicated on some recently inferred phylogenies; see Fig. 3), it is perhaps appropriate to express

2414 some skepticism towards this inference. As already noted by Müller et al. (2018), the originally

2415 proposed tetanurine affinities have not been tested by Baron \& Barrett $(2017,2018)$, nor by

2416 Baron (2019), or Müller \& Dias-da-Silva (2019), all of whom have also reconstructed $C$.

2417 diegosuarezi at the earliest-diverging position within Ornithischia. It is worth noting that

2418 Dieudonné et al. (2020) included C. diegosuarezi in their data matrix as well; however, despite

2419 being considered an ornithischian by the authors, its placement at the very base of their tree does

2420 not indicate ornithischian affinities for the taxon. Dieudonné et al. (2020) did not include any

2421 theropods and/or sauropodomorphs in their analysis and, as such, they did not explore the

2422 placement of $C$. diegosuarezi among dinosaurs. In turn, the studies of Baron \& Barrett (2017,

2423 2018), Baron (2019), and Müller \& Dias-da-Silva (2019) have all been based on a dataset

2424 modified from the one first published by Baron et al. (2017a) that was constructed to test the

2425 relationships of rootward dinosaurs (ornithischians, theropods, and sauropodomorphs), especially

2426 Late Triassic and Early Jurassic forms (though some younger taxa of Ornithischia have been

2427 included as well) and their closest pan-avian relatives. We are of the opinion that, at present and 
2428 with the evidence provided, the placement of Chilesaurus, a latest Jurassic taxon with mosaic

2429 features, at the very base of a clade that has originated in the Triassic or at the Triassic/Jurassic

2430 boundary interval, may be best interpreted as being indicative of inadequate/inappropriate data

2431 sampling. In other words, the dataset of Baron et al. (2017a) and its more recent versions are

2432 most likely unable to actually test the phylogenetic placement of Chilesaurus.

2433

2434

\section{The phylogenetic placement of Heterodontosauridae}

The members of Heterodontosauridae have long been treated as early-diverging ornithopods (e.g., Sereno, 1986, 1998, 1999). The last two decades have shown, however, that heterodontosaurids represent some of the more problematic ornithischian groups; with some studies inferring them as non-ornithopod neornithischians (Butler, 2005), as the sister group to Marginocephalia (Xu et al., 2006), near the base of Ornithischia (e.g., Butler et al., 2008; Boyd, 2015; Sereno, 2012; Dieudonné et al., 2016; Han et al., 2018; Madzia et al., 2018; Andrzejewski et al., 2019; Herne et al., 2019; Yang et al., 2020), and within Pachycephalosauria (Dieudonné et al., 2020). With respect to the recent reconstruction of heterodontosaurids as early-diverging pachycephalosaurs by Dieudonné et al. (2020), it is worth noting that Heterodontosauridae still form a clade (contra Dieudonné et al., 2020). Even though some taxa that are usually inferred as members of Heterodontosauridae (Echinodon becklesii and Tianyulong confuciusi) are placed more closely to pachycephalosaurids in Dieudonné et al. (2020: Fig. 1) than to Heterodontosaurus, making the 'traditional' composition of the group as inferred in other recent studies paraphyletic, Heterodontosauridae still comprises Abrictosaurus consors, Fruitadens haagarorum, Heterodontosaurus tucki, and Lycorhinus angustidens in that study. Similarly, under the topology of Xu et al. (2006), heterodontosaurids and marginocephalians were inferred as the sister taxa, forming a clade named Heterodontosauriformes. Such topology has not been supported in more recent studies (see studies cited above).

2453 Regardless of which of the hypotheses will gain further support in subsequent studies, the definition of the name Heterodontosauridae needs to reflect each of them. Therefore, the applied phylogenetic definition of the name includes representatives of all major ornithischian lineages, Ceratopsia (Triceratops horridus), Ornithopoda (Iguanodon bernissartensis), stenops). 


\section{The early-diverging thyreophorans and ankylosaurs}

2461 The 'armored' dinosaurs, Thyreophora, comprise two major clades, Ankylosauria and

2462 Stegosauria, and other taxa that are more closely related to members of the two species-rich

2463 lineages than to ornithopods and marginocephalians. These include Emausaurus ernsti,

2464 Scelidosaurus harrisonii, and Scutellosaurus lawleri (Han et al., 2018; Herne et al., 2019;

2465 Madzia et al., 2018; Dieudonné et al., 2020), and some other, more problematic taxa, such as the

2466 dubious 'Tatisaurus oehleri' (Norman et al., 2007) and 'Bienosaurus lufengensis' (Raven et al.,

2467 2019). Additionally, Lesothosaurus diagnosticus and Laquintasaura venezuelae have been

2468 inferred as early-diverging thyreophorans as well (see, e.g., Butler et al., 2008 for the placement

2469 of L. diagnosticus, and, e.g., Baron et al., 2017c and Andrzejewski et al., 2019 for the position of

2470 L. venezuelae). Other studies, however, place Le. diagnosticus as an early neornithischian

2471 (Madzia et al., 2018; Herne et al., 2019) or an early-diverging ornithischian in general (Han et

2472 al., 2018; Andrzejewski et al., 2019; Dieudonné et al., 2020; Yang et al., 2020), and La.

2473 venezuelae as an early-diverging ornithischian (Han et al., 2018; Dieudonné et al., 2020; Yang et

2474 al., 2020).

2475 Following his thorough redescription of Scelidosaurus harrisonii (Norman, 2020a, 2020b,

2476 2020c), Norman (2021) assessed the phylogenetic relationships of early-diverging thyreophorans

2477 and reconstructed E. ernsti, Sce. harrisonii, and Scu. lawleri as the earliest-diverging

2478 ankylosauromorphs (Ankylosauria sensu this study), restricting the name Ankylosauria to a

2479 smaller clade, approximately comprising ankylosaurids and nodosaurids (two definitions - one

2480 minimum-clade and one maximum-clade - were provided; both applying the name to the same

2481 known contents). Norman (2021: 70) further noted that the node comprising ankylosaurids and

2482 nodosaurids "has the potential to become the new taxon Euankylosauria but this additional clade

2483 name is neither essential nor particularly desirable".

2484 When applying a minimum-clade definition (e.g., 'min $\nabla$ (Ankylosaurus magniventris Brown,

24851908 \& Nodosaurus textilis Marsh, 1889)'), the name Euankylosauria may indeed be useful in

2486 the future, especially if further studies support the placement of some taxa, such as

2487 Mymoorapelta maysi and Kunbarrasaurus ieversi (as in Arbour and Currie, 2016), or E. ernsti,

2488 Sce. harrisonii, and Scu. lawleri (as in Norman, 2020c), as non-ankylosaurid/non-nodosaurid

2489 ankylosaurs. However, there is no need to replace Ankylosauria with Ankylosauromorpha as the 
2490 name for the largest clade containing A. magniventris but not Stegosaurus stenops. The branch

2491 has long been named Ankylosauria and it has always been expected that it may contain taxa with 2492 characters that are absent in 'traditional' ankylosaurs (i.e., ankylosaurids and nodosaurids). We 2493 suggest that the name Ankylosauromorpha is abandoned.

\section{Problematic clades within Ankylosauria}

Comprehensive alpha taxonomic reviews and phylogenetic analyses of Ankylosauridae in recent years have clarified many of the interrelationships within this clade (e.g., Arbour \& Currie 2013; Arbour \& Currie 2016). However, similar reviews for Nodosauridae have not been undertaken in recent years, and phylogenetic resolution within Nodosauridae is often poor and inconsistent between different phylogenies (e.g., Thompson et al., 2012; Arbour et al., 2016; Brown et al., 2017), in part because many recent ankylosaur phylogenetic analyses are modified from Arbour \& Currie (2016) which was designed to test relationships within Ankylosauridae, not Nodosauridae. Additionally, many names for clades within Nodosauridae have been introduced by various authors based on proposed diagnostic characters rather than phylogenetic hypotheses, and have not been defined phylogenetically. In particular, the validity of Polacanthidae or Polacanthinae, Sauropeltinae, Struthiosaurinae, and Stegopeltinae, and the contents of Edmontiniinae or Panoplosaurinae, are unclear. In this manuscript we provide a formal definition of Polacanthinae, and discuss the use of Struthiosaurinae and Panoplosaurinae, as the names have been mentioned recently with some frequency and have had informal definitions proposed previously. Ford (2000) introduced the names Sauropeltinae and Stegopeltinae and provided diagnostic characters but did not test their contents phylogenetically; Sauropeltinae included Sauropelta edwardsorum and Silvisaurus condrayi and Stegopeltinae included Aletopelta coombsi, Glyptodontopelta mimus, and Stegopelta landerensis. Sauropelta and

2514 Silvisaurus do not form a clade in any recent analyses, nor do Stegopelta, Glyptodontopelta, and 2515 Aletopelta. As such, we do not provide formal definitions for Sauropeltinae or Stegopeltinae at 2516 this time.

\section{The origin of Ornithopoda}

The understanding of the origin and early evolution of Ornithopoda is tightly connected with the 
2521 basal neornithischian-ornithopod transition is among the poorest known stages of the 2522 ornithischian evolutionary history, as recent phylogenetic studies that focused on that particular 2523 tree segment provided strikingly conflicting topologies (Boyd, 2015; Dieudonné et al., 2016; 2524 Han et al., 2018; Madzia et al., 2018; Andrzejewski et al., 2019; Herne et al., 2019; Dieudonné et 2525 al., 2020; Yang et al., 2020).

2526 Substantial conflicts are apparent especially with regards to the phylogenetic placements of taxa 2527 'traditionally' dubbed the 'hypsilophodonts' (compare, e.g., Boyd, 2015; Han et al. 2018; Herne 2528 et al., 2019), including Hypsilophodon foxii itself (e.g., Madzia et al., 2018). Phylogeny 2529 reconstructions of ornithopods provide more stable results around the node marking the origin of 2530 Iguanodontia (e.g., Madzia et al., 2018; Madzia et al., 2020), although alternative hypotheses of 2531 early iguanodontian phylogenetic relationships exist as well (e.g., Norman, 2015). The names of 2532 non-cerapod neornithischian and rootward ornithopod clades are defined here to reflect these 2533 uncertainties though we recognize that some potential topologies may still render issues. For 2534 example, if Hypsilophodon forms a clade with thescelosaurids but falls outside the 2535 Thescelosaurus + Orodromeus node, Hypsilophodontidae would cover Thescelosauridae if the 2536 latter name was defined using a minimum-clade definition (as in Brown et al., 2013 and Madzia 2537 et al., 2018). We do not include T. neglectus as an external specifier in the definition of 2538 Hypsilophodontidae because under the scenario, in which $H$. foxii would be inferred within the Thescelosaurus + Orodromeus node, the names Thescelosauridae, Thescelosaurinae, and Orodrominae would be all inapplicable, while Hypsilophodontidae could effectively remain in use only for $H$. foxii. The definitions we propose ensure that if $H$. foxii is component of the Thescelosaurus + Orodromeus clade, Thescelosauridae becomes inapplicable, while Thescelosaurinae and Orodrominae still remain in use. The potential issue with Hypsilophodontidae covering Thescelosauridae under a topology in which Hypsilophodon is the sister taxon to the Thescelosaurus + Orodromeus node was solved by providing 2547 scenario.

\section{Hadrosaurid ingroup relationships}

2550 Hadrosaurids are some of the most intensively researched ornithischians, with thoroughly explored phylogenetic relationships. Recent studies almost uniformly infer seven major 
2552 hadrosaurid clades: Brachylophosaurini, Edmontosaurini, Kritosaurini, Lambeosaurini,

2553 Parasaurolophini, Saurolophini, and Tsintaosaurini (e.g., Freedman Fowler \& Horner, 2015;

2554 Prieto-Márquez et al., 2016; Xing et al., 2017; Kobayashi et al., 2019; Prieto-Márquez et al.,

2555 2019; Prieto-Márquez et al., 2020; Zhang et al., 2020; Kobayashi et al., 2021; Longrich et al.,

2556 2021; McDonald et al., 2021; Ramírez-Velasco et al., 2021). Longrich et al. (2021) recently

2557 introduced a new clade name, Arenysaurini, for a diverse grouping of mostly European

2558 lambeosaurines, resulting, at the same time, in that Tsintaosaurini (as originally used and as

2559 defined here) becomes inapplicable. The study of Longrich et al. (2021) was first to infer such

2560 topology. Other phylogenetic studies placed Arenysaurus ardevoli either deeply within

2561 Lambeosaurini (e.g., Prieto-Márquez et al., 2016; Prieto-Márquez et al., 2019; Zhang et al.,

2562 2019; Prieto-Márquez et al., 2020; Gates et al., 2021; Ramírez-Velasco et al., 2021), within

2563 Parasaurolophini (Cruzado-Caballero et al., 2013), or as the sister taxon or close to the clade

2564 uniting Lambeosaurini and Parasaurolophini (e.g., Pereda-Suberbiola et al., 2009; Cruzado-

2565 Caballero et al., 2010; Godefroit et al., 2012; Cruzado-Caballero \& Powell, 2017; Xing et al.,

2566 2017; Kobayashi et al., 2019; Zhang et al., 2020).

2567 Owing to the fact that the consensus regarding the placement of Arenysaurus ardevoli among

2568 lambeosaurines has yet to be reached, and that other 'arenysaurins' of Longrich et al. (2021) are

2569 distributed across the lambeosaurine tree in other studies, we do not define Arenysaurini here. If

2570 future studies support the results of Longrich et al. (2021), Arenysaurini should probably be

2571 defined so that it becomes inapplicable if inferred within Lambeosaurini. The easiest way to

2572 obtain such effect would be to define Arenysaurini through a maximum-clade definition using

2573 Arenysaurus ardevoli and at least one another internal specifier that would make the name

2574 applicable only in the case Arenysaurus is inferred outside Lambeosaurini. The taxon

2575 Adynomosaurus arcanus is a possible candidate, if such solution is preferred. In turn,

2576 Blasisaurus canudoi should be avoided as this taxon has been inferred as the sister taxon of $A$.

2577 ardevoli in some analyses (e.g., Cruzado-Caballero et al., 2010; Cruzado-Caballero et al., 2013;

2578 Prieto-Márquez et al., 2019; Prieto-Márquez et al., 2020; Gates et al., 2021). Another option is to

2579 apply a clause similar to that we used in the definitions of Clypeodonta, Euornithopoda,

2580 Hypsilophodontia, Orodrominae, and Thescelosaurinae. That is, by using the set theory symbol

$2581 \notin$, meaning “not element of”, the name Arenysaurini could be applicable only under the

2582 condition that the clade for which the name was intended was reconstructed outside

Peer] reviewing PDF | (2021:04:60693:1:1:NEW 8 Sep 2021) 
2583 Lambeosaurini and Parasaurolophini. Such definition could be abbreviated as follows: $\max \nabla \notin$

2584 Lambeosaurini \& Parasaurolophini (Arenysaurus ardevoli Pereda-Suberbiola et al., 2009

2585 Lambeosaurus lambei Parks, 1923 \& Parasaurolophus walkeri Parks, 1922).

2586

2587 Conclusions

2588 Ornithischian dinosaurs were a major clade of globally distributed Mesozoic archosaurs that 2589 achieved substantial taxic diversity and apparent morphological disparity, expressed especially

2590 through their cranial features and the body armor of some of their most distinctive members.

2591 Throughout their two-century-long reasearch history, ornithischians have been thoroughly

2592 assessed both taxonomically and phylogenetically, which have led to the recognition of

2593 numerous clades.

2594 Following the pivotal studies establishing the theoretical foundation of the phylogenetic

2595 nomenclature in the 1980s and early 1990s, many names for the ornithischian clades have been

2596 provided phylogenetic definitions, some of which have proven useful and have not been changed

2597 since their introduction.

2598 However, following the 2020 establishment of the International Code of Phylogenetic

2599 Nomenclature $(I C P N)$, or the PhyloCode, all of the definitions proposed before the

2600 implementation of the Code are treated as formally ineffective.

2601 We have reconsidered the utility of previously proposed phylogenetic definitions of established 2602 ornithischian taxon names and provide definitions for 81 names of ornithischian clades, five of 2603 which are newly proposed here, as specified by the Articles of the ICPN, thus marking a key step 2604 towards a formal phylogenetic nomenclature of ornithischian dinosaurs.

2605

2606 Acknowledgements

2607 We would like to express our gratitude to Academic Editor Fabien Knoll (ARAID-Fundación

2608 Conjunto Paleontológico de Teruel-Dinópolis, Teruel, Spain) for handling our manuscript, and 2609 Paul M. Barrett (Natural History Museum, London, England), Thomas R. Holtz (University of 2610 Maryland, MD, USA), and Max C. Langer (Universidade de São Paulo, São Paulo, Brazil) for 2611 their detailed reviews that improved the manuscript.

2612

2613 Funding

Peer) reviewing PDF | (2021:04:60693:1:1:NEW 8 Sep 2021) 
2614 The study was funded by the National Science Centre (Poland) grant no. 2020/37/B/NZ8/01321 2615 to Daniel Madzia (Institute of Paleobiology, Polish Academy of Sciences). VMA is funded by a 2616 Natural Sciences and Engineering Research Council Discovery Grant (RGPIN-2020-04012).

\section{References}

2619

2620

2621

2622

2623

2624

2625

2626

2627

Abel OLFAL. 1919. Die Stämme der Wirbeltiere, Berlin und Leipzig. W. de Gruyter, 914 pp. Agnolin FL, Ezcurra MD. 2019. The Validity of Lagosuchus talampayensis Romer, 1971 (Archosauria, Dinosauriformes), from the Late Triassic of Argentina. Breviora 565: 1-21. Agnolín FL, Rozadilla S. 2018. Phylogenetic reassessment of Pisanosaurus mertii Casamiquela, 1967, a basal dinosauriform from the Late Triassic of Argentina. Journal of Systematic Palaeontology 16: 853-879.

Andrzejewski KA, Winkler DA, Jacobs LL. 2019. A new basal ornithopod (Dinosauria: Ornithischia) from the Early Cretaceous of Texas. PLoS ONE 14: e0207935. Anonymous. 1865. A new Wealden dragon. Order, Sauria; Family, Dinosaurian; Genus, Polacanthus; Species, foxii. Illustrated London News: 47.

Arbour VM, Currie PJ. 2013. Euoplocephalus tutus and the diversity of ankylosaurid dinosaurs in the Late Cretaceous of Alberta, Canada, and Montana, USA. PLoS ONE 8: e62421.

Arbour VM, Currie PJ. 2016. Systematics, phylogeny and palaeobiogeography of the ankylosaurid dinosaurs. Journal of Systematic Palaeontology 14: 385-444.

Arbour VM, Evans DC. 2019. A new leptoceratopsid dinosaur from Maastrichtian-aged deposits of the Sustut Basin, northern British Columbia, Canada. PeerJ 7: e7926.

Arbour VM, Zanno LE, Gates T. 2016. Ankylosaurian dinosaur palaeoenvironmental associations were influenced by extirpation, sea-level fluctuation, and geodispersal. Palaeogeography, Palaeoclimatology, Palaeoecology 449: 289-299.

Bakker RT. 1988. Review of the Late Cretaceous nodosaurid Dinosauria: Denversaurus schlessmani, a new armor-plated dinosaur from the latest Cretaceous of South Dakota, the last survivor of the nodosaurians, with comments on stegosaur-nodosaur relationships. Hunteria 1: 1-23.

Baron MG. 2019. Pisanosaurus mertii and the Triassic ornithischian crisis: could phylogeny offer a solution? Historical Biology 31: 967-981. 
2644 Baron MG, Barrett PM. 2017. A dinosaur missing-link? Chilesaurus and the early evolution of 2645 ornithischian dinosaurs. Biology Letters 13: 20170220.

2646 Baron MG, Barrett PM. 2018. Support for the placement of Chilesaurus within Ornithischia: a 2647 reply to Müller et al. Biology Letters 14: 20180002.

2648 Baron MG, Norman DB, Barrett PM. 2017a. A new hypothesis of dinosaur relationships and 2649 early dinosaur evolution. Nature 543: 501-506.

Baron MG, Norman DB, Barrett PM. 2017b. Baron et al. reply. Nature 551: E4-E5.

Baron MG, Norman DB, Barrett PM. 2017c. Postcranial anatomy of Lesothosaurus diagnosticus (Dinosauria: Ornithischia) from the Lower Jurassic of southern Africa: implications for basal ornithischian taxonomy and systematics. Zoological Journal of the Linnean Society 179: 125-168.

Barta DE, Norell MA. 2021. The osteology of Haya griva (Dinosauria: Ornithischia) from the Late Cretaceous of Mongolia. Bulletin of the American Museum of Natural History 445: 1-111.

Baur G. 1891. Remarks on the reptiles generally called Dinosauria. American Naturalist 25: 434-454.

Benson RBJ, Hunt G, Carrano MT, Campione N. 2018. Cope's rule and the adaptive landscape of dinosaur body size evolution. Palaeontology 61: 13-48.

Blows WT, Honeysett K. 2014. First Valanginian Polacanthus foxii (Dinosauria, Ankylosauria) from England, from the Lower Cretaceous of Bexhill, Sussex. Proceedings of the Geologists' Association 125: 233-251.

Bonaparte C-L. 1850. Conspectus Systematum Herpetologiae et Amphibiologiae. Editio Altera Reformata. E. J. Brill, Leyden 1.

Boulenger GA. 1881. Iguanodon bernissartensis. p. 606. In Beneden P-J.. Sur l'arc pelvien chex les dinosauriens de Bernissart. Bulletin de l'Académie royal de Belgique 3 Sér. 1: 600608.

Boyd CA. 2015. The systematic relationships and biogeographic history of ornithischian dinosaurs. PeerJ 3: e1523.

Brett-Surman MK. 1989. A revision of the Hadrosauridae (Reptilia: Ornithischia) and their evolution during the Campanian and Maastrichtian. Unpublished PhD thesis, George Washington University, $192 \mathrm{pp}$. 
2675 Brown B. 1908. The Ankylosauridae, a new family of armored dinosaurs from the Upper

2676

2677

2678

2679

2680

2681

2682

2683

2684

2685

2686

2687

2688

2689

2690

2691

2692

2693

2694

2695

2696

2697

2698

2699

2700

2701

2702

2703

Cretaceous. Bulletin of the American Museum of Natural History 24: 187-201.

Brown B. 1910. The Cretaceous Ojo Alamo beds of New Mexico with description of the new dinosaur genus Kritosaurus. Bulletin of the American Museum of Natural History 28: 267274.

Brown B. 1912. A crested dinosaur from the Edmonton Cretaceous. Bulletin of the American Museum of Natural History 31: 131-136.

Brown B. 1914a. Corythosaurus casuarius, a New Crested Dinosaur from the Belly River Cretaceous, with Provisional Classification of the Family Trachodontidae. American Museum of Natural History Bulletin 33: 559-565.

Brown B. 1914b. Leptoceratops, a new genus of Ceratopsia from the Edmonton Cretaceous of Alberta. Bulletin of the American Museum of Natural History 33: 567-580.

Brown B. 1914c. Anchiceratops, a new genus of horned dinosaurs from the Edmonton Cretaceous of Alberta. With discussion of the origin of the ceratopsian crest and the brain casts of Anchiceratops and Trachodon. Bulletin of the American Museum of Natural History 33: 539-548.

Brown CM. 2017. An exceptionally preserved armored dinosaur reveals the morphology and allometry of osteoderms and their horny epidermal coverings. PeerJ 5: e4066.Butler RJ. 2005. The 'fabrosaurid' ornithischian dinosaurs of the Upper Elliot Formation (Lower Jurassic) of South Africa and Lesotho. Zoological Journal of the Linnean Society 145: $175-218$.

Brown CM, Druckenmiller P. 2011. Basal ornithopod (Dinosauria: Ornithischia) teeth from the Prince Creek Formation (early Maastrichtian) of Alaska. Canadian Journal of Earth Sciences 48: 1342-1354.

Brown CM, Evans DC, Ryan MJ, Russell AP. 2013. New data on the diversity and abundance of small-bodied ornithopods (Dinosauria, Ornithischia) from the Belly River Group (Campanian) of Alberta. Journal of Vertebrate Paleontology 33: 495-520.

Brown CM, Henderson DM. 2015. A new horned dinosaur reveals convergent evolution in cranial ornamentation in Ceratopsidae. Current Biology 25: 1641-1648.

Peer) reviewing PDF | (2021:04:60693:1:1:NEW 8 Sep 2021) 
2704 Brown CM, Henderson DM, Vinther J, Fletcher I, Sistiaga A, Herrera J, Summons RE. 2017. An

2705

2706

2707

2708

2709

2710

2711

2712

2713

2714

2715

2716

2717

2718

2719

2720

2721

2722

2723

2724

2725

2726

2727

2728

2729

2730

2731

2732

2733

2734
Exceptionally Preserved Three-Dimensional Armored Dinosaur Reveals Insights into

Coloration and Cretaceous Predator-Prey Dynamics. Current Biology 27: 2514-2521.e3.

Brusatte SL, Butler RJ, Barrett PM, Carrano MT, Evans DC, Lloyd GT, Mannion PD, Norell

MA, Peppe DJ, Upchurch P, Williamson TE. 2015. The extinction of the dinosaurs.

Biological Reviews 90: 628-642.

Buchholz PW. 2002. Phylogeny and biogeography of basal Ornithischia. In Brown ED (ed.). The Mesozoic of Wyoming. Proceedings of the Tate Museum Geology Conference 2002: 1834.

Bunzel E. 1871. Die Reptilfauna der Gosauformation in der Neuen Welt bei Weiner-Neustadt. Abhandlungen der Kaiserlich-Königlichen Geologischen Reichsanstalt 5: 1-18.

Burns ME. 2015. Intraspecific variation in the armoured dinosaurs (Dinosauria: Ankylosauria).

PhD Dissertation, University of Alberta, $285 \mathrm{pp}$.

Butler RJ. 2005. The 'fabrosaurid' ornithischian dinosaurs of the Upper Elliot Formation (Lower Jurassic) of South Africa and Lesotho. Zoological Journal of the Linnean Society 145: $175-218$.

Butler RJ, Upchurch P, Norman DB. 2008. The phylogeny of the ornithischian dinosaurs. Journal of Systematic Palaeontology 6: 1-40.

Cabreira SF, Kellner AWA, Dias-da-Silva S, da Silva LR, Bronzati M, de Almeida Marsola JC, Müller RT, de Souza Bittencourt J, Batista BJ, Raugust T, Carrilho R, Brodt A, Langer MC. 2016. A Unique Late Triassic Dinosauromorph Assemblage Reveals Dinosaur Ancestral Anatomy and Diet. Current Biology 26: 3090-3095.

Calvo JO, Porfiri JD, Novas FE. 2007. Discovery of a new ornithopod dinosaur from the Portezuelo Formation (Upper Cretaceous), Neuquén, Patagonia, Argentina. Arquivos do Museu Nacional, Rio de Janeiro 65: 471-483.

Campbell JA, Ryan MJ, Schröder-Adams CJ, Holmes RB, Evans DC. 2019. Temporal range extension and evolution of the chasmosaurine ceratopsid 'Vagaceratops' irvinensis (Dinosauria: Ornithischia) in the Upper Cretaceous (Campanian) Dinosaur Park Formation of Alberta. Vertebrate Anatomy Morphology Palaeontology 7: 83-100.

Carpenter K. 1997. Ankylosauria. In Currie PJ, Padian K (eds). Encyclopedia of Dinosaurs. Academic Press, San Diego: 16-20.

Peer) reviewing PDF | (2021:04:60693:1:1:NEW 8 Sep 2021) 
2735 Carpenter K. 2001. Phylogenetic analysis of the Ankylosauria. In Carpenter K (ed.). The

2736

2737

2738

2739

2740

2741

2742

2743

2744

2745

2746

2747

2748

2749

2750

2751

2752

2753

2754

2755

2756

2757

2758

2759

2760

2761

2762

2763

2764

2765

Armored Dinosaurs. Indiana University Press, Bloomington: 455-483.

Carpenter K, Galton PM. 2018. A photo documentation of bipedal ornithischian dinosaurs from the Upper Jurassic Morrison Formation, USA. Geology of the Intermountain West 5: 167207.

Carpenter K, Lamanna MC. 2015. The Braincase Assigned to the Ornithopod Dinosaur Uteodon McDonald, 2011, Reassigned to Dryosaurus Marsh, 1894: Implications for Iguanodontian Morphology and Taxonomy. Annals of Carnegie Museum 83: 149-165.

Casamiquela RM. 1967. Un nuevo dinosaurio ornitisquio triasico (Pisanosaurus mertii;

Ornithopoda) de la Formación Ischigualasto, Argentina. Ameghiniana 4: 47-64.

Casanovas-Cladellas ML, Santafé-Llopis JV, Isidro-Llorens A. 1993. Pararhabdodon isonensis n. gen. n. sp. (Dinosauria). Estudio mofológico, radio-tomográfico y consideraciones biomecanicas. Paleontologia i Evolució 26-27: 121-131.

Cau A. 2018. The assembly of the avian body plan: a 160-million-year long process. Bollettino della Società Paleontologica Italiana 57: 1-25.

Chiba K, Ryan MJ, Fanti F, Loewen MA, Evans DC. 2018. New material and systematic reevaluation of Medusaceratops lokii (Dinosauria, Ceratopsidae) from the Judith River Formation (Campanian, Montana). Journal of Paleontology 92: 272-288.

Cooper MR. 1985. A revision of the ornithischian dinosaur Kangnasaurus coetzeei Haughton, with a classification of the Ornithischia. Annals of the South African Museum 95: 281317.

Cope ED. 1869. Synopsis of the Extinct Batrachia, Reptilia and Aves of North America. Part I. Transactions of the American Philosophical Society, New Series 14: 1-104.

Cope ED. 1877. On a gigantic saurian from the Dakota epoch of Colorado. Paleontological Bulletin 25: 5-10.

Coria RA, Salgado L. 1996. A basal iguanodontian (Ornithischia: Ornithopoda) from the Late Cretaceous of South America. Journal of Vertebrate Paleontology 16: 445-457.

Crompton AW, Charig AJ. 1962. A new ornithischian from the Upper Triassic of South Africa. Nature 196: 1074-1077.

Cruzado-Caballero P, Canudo JI, Moreno-Azanza M, Ruiz-Omeñaca JI. 2013. New material and phylogenetic position of Arenysaurus ardevoli, a lambeosaurine dinosaur from the late

Peer) reviewing PDF | (2021:04:60693:1:1:NEW 8 Sep 2021) 
2766

2767

2768

2769

2770

2771

2772

2773

2774

2775

2776

2777

2778

2779

2780

2781

2782

2783

2784

2785

2786

2787

2788

2789

2790

2791

2792

2793

2794

2795

Maastrichtian of Arén (northern Spain). Journal of Vertebrate Paleontology 33: 13671384.

Cruzado-Caballero P, Pereda-Suberbiola X, Ruiz-Omeñaca JI. 2010. Blasisaurus canudoi gen. et sp. nov., a new lambeosaurine dinosaur (Hadrosauridae) from the latest Cretaceous of Arén (Huesca, Spain). Canadian Journal of Earth Sciences 47: 1507-1517.

Cruzado-Caballero P, Powell J. 2017. Bonapartesaurus rionegrensis, a new hadrosaurine dinosaur from South America: implications for phylogenetic and biogeographic relations with North America, Journal of Vertebrate Paleontology 37: e1289381.

Currie PJ, Padian K. 1997. Cerapoda. In Currie PJ, Padian K. (eds.). Encyclopedia of Dinosaurs. Academic Press, San Diego: 105.

Czepiński Ł. 2020. Ontogeny and variation of a protoceratopsid dinosaur Bagaceratops rozhdestvenskyi from the Late Cretaceous of the Gobi Desert. Historical Biology 32: 1394 1421.

Černý D, Madzia D, Slater GJ. 2021. Empirical and Methodological Challenges to the ModelBased Inference of Diversification Rates in Extinct Clades. Systematic Biology. doi:10.1093/sysbio/syab045

Dalman SG, Hodnett J-PM, Lichtig, AJ, Lucas SG. 2018. A new ceratopsid dinosaur (Centrosaurinae: Nasutoceratopsini) from the Fort Crittenden Formation, Upper Cretaceous (Campanian) of Arizona. New Mexico Museum of Natural History and Science Bulletin 79: 141-164.

Dalman SG, Lucas SG, Jasinki SG, Lichtig AJ, Dodson P. 2021. The oldest centrosaurine: a new ceratopsid dinosaur (Dinosauria: Ceratopsidae) from the Allison Member of the Menefee Formation (Upper Cretaceous, early Campanian), northwestern New Mexico, USA. PalZ 95: 291-335.

de Queiroz K. 1988. Systematics and the Darwinian revolution. Philosophy of Science 55: 238259.

de Queiroz K, Cantino PD (eds). 2020. International Code of Phylogenetic Nomenclature (PhyloCode). CRC Press, 189 pp.

de Queiroz K, Cantino PD, Gauthier JA (eds). 2020. Phylonyms. A Companion to the PhyloCode. CRC Press, 1352 pp. 
2796 de Queiroz K, Gauthier J. 1990. Phylogeny as a central principle in taxonomy: phylogenetic 2797 definitions of taxon names. Systematic Zoology 39: 307-322.

2798 de Queiroz K, Gauthier J. 1992. Phylogenetic taxonomy. Annual Review of Ecology and 2799 Systematics 23: 449-480.

2800 de Queiroz K, Gauthier J. 1994. Toward a phylogenetic system of biological nomenclature. 2801 Trends in Ecology and Evolution 9: 27-31.

2802 Desojo JB, Fiorelli LE, Ezcurra MD, Martinelli AG, Ramezani J, Da Rosa AAS, Belén von 2803 Baczko M, Trotteyn MJ, Montefeltro FC, Ezpeleta M, Langer MC. 2020. The Late Triassic 2804 2805 2806 2807 2808 Ischigualasto Formation at Cerro Las Lajas (La Rioja, Argentina): fossil tetrapods, highresolution chronostratigraphy, and faunal correlations. Scientific Reports 10: 12782.

Dieudonné PE, Cruzado-Caballero P, Godefroit P, Tortosa T. 2020. A new phylogeny of cerapodan dinosaurs. Historical Biology. https://doi.org/10.1080/08912963.2020.1793979

Dieudonné P-E, Tortosa T, Torcida Fernández-Baldor F, Canudo JI, Díaz-Martínez I. 2016. An Unexpected Early Rhabdodontid from Europe (Lower Cretaceous of Salas de los Infantes, Burgos Province, Spain) and a Re-Examination of Basal Iguanodontian Relationships. PLoS ONE 11: e0156251.

Dodson P. 1997. Ceratopsia. In Currie PJ, Padian K. (eds.). Encyclopedia of Dinosaurs. Academic Press, San Diego: 106.

Dodson P, Forster CA, Sampson SD. 2004. Ceratopsidae. In Weishampel DB, Dodson P, Osmólska H. (eds). The Dinosauria. 2nd Ed. University of California Press, Berkeley: 494 513.

Dollo L. 1882. Première note sur les dinosaures de Bernissart. Bulletin du Musée Royale d'Histoire Naturelle de Belgique 1: 1-18.

Dong Z, Tang Z, Zhou SW. 1982. [Note on the new Mid-Jurassic stegosaur from Sichuan Basin, China]. Vertebrata PalAsiatica 20: 83-87. (in Chinese)

Dzik J. 2003. A beaked herbivorous archosaur with dinosaur affinities from the early Late Triassic of Poland. Journal of Vertebrate Paleontology 23: 556-574.

Estes R, de Queiroz K, Gauthier J. 1988. Phylogenetic relationships within Squamata. In Estes R, Pregill GK. (eds). Phylogenetic Relationships of the Lizard Families: Essays Commemorating Charles L. Camp. Stanford University Press, Stanford, California: 119281. 
2827 Evans DC, Reisz RR. 2007. Anatomy and relationships of Lambeosaurus magnicristatus, a 2828 crested hadrosaurid dinosaur (Ornithischia) from the Dinosaur Park Formation, Alberta. 2829 Journal of Vertebrate Paleontology 27: 373-393.

2830 Evans DC, Schott RK, Larson DW, Brown CM, Ryan MJ. 2013. The oldest North American 2831 pachycephalosaurid and the hidden diversity of small-bodied ornithischian dinosaurs. $2832 \quad$ Nature Communications 4: 1828.

2833 Ezcurra MD. 2016. The phylogenetic relationships of basal archosauromorphs, with an emphasis on the systematics of proterosuchian archosauriforms. PeerJ 4: e1778.

Ezcurra MD, Nesbitt SJ, Bronzati M, Dalla Vecchia FM, Agnolin FL, Benson RBJ, Brissón Egli F, Cabreira SF, Evers SW, Gentil AR, Irmis RB, Martinelli AG, Novas FE, da Silva LR, Smith ND, Stocker MR, Turner AH, Langer MC. 2020. Enigmatic dinosaur precursors bridge the gap to the origin of Pterosauria. Nature 588: 445-449.

Fabbri M, Tschopp E, McPhee B, Nesbitt S, Pol D, Langer M. 2020. Sauropodomorpha. In de Queiroz K, Cantino PD, Gauthier JA (eds). Phylonyms. A Companion to the PhyloCode. CRC Press: 1225-1234.

Fiorillo AR, Tykoski RS. 2012. A new Maastrichtian species of the centrosaurine ceratopsid Pachyrhinosaurus from the North Slope of Alaska. Acta Palaeontologica Polonica 57: 561-573.

Ford TL. 2000. A review of ankylosaur osteoderms from New Mexico and a preliminary review of ankylosaur armor. New Mexico Museum of Natural History and Science Bulletin 17: $157-176$.

Fowler DW. 2017. Revised geochronology, correlation, and dinosaur stratigraphic ranges of the Santonian-Maastrichtian (Late Cretaceous) formations of the Western Interior of North America. PLoS ONE 12: e0188426.

Fowler DW, Freedman Fowler EA. 2020. Transitional evolutionary forms in chasmosaurine ceratopsid dinosaurs: evidence from the Campanian of New Mexico. PeerJ 8: e9251.

Freedman Fowler EA, Horner JR. 2015. A New Brachylophosaurin Hadrosaur (Dinosauria: Ornithischia) with an Intermediate Nasal Crest from the Campanian Judith River Formation of Northcentral Montana. PLoS ONE 10: e0141304.

Galton PM. 1997. Stegosauria. In Currie PJ, Padian K. (eds.). Encyclopedia of Dinosaurs. Academic Press, San Diego: 701-703. 
2858

2859

2860

2861

2862

2863

2864

2865

2866

2867

2868

2869

2870

2871

2872

2873

2874

2875

2876

2877

2878

2879

2880

2881

2882

2883

2884

2885

2886

2887

2888

Galton PM. 2010. Species of plated dinosaur Stegosaurus (Morrison Formation, Late Jurassic) of western USA: new type species designation needed. Swiss Journal of Geosciences 103: 187-198.

Galton PM, Upchurch P. 2004. Stegosauria. In Weishampel DB, Dodson P, Osmólska H. (eds). The Dinosauria, 2nd Ed). Berkeley: University of California Press: 343-362.

Gates TA, Evans DC, Sertich JJW. 2021. Description and rediagnosis of the crested hadrosaurid (Ornithopoda) dinosaur Parasaurolophus cyrtocristatus on the basis of new cranial remains. PeerJ 9: e10669.

Gates TA, Horner JR, Hanna RR, Nelson CR. 2011. New unadorned hadrosaurine hadrosaurid (Dinosauria, Ornithopoda) from the Campanian of North America. Journal of Vertebrate Paleontology 31: 798-811.

Gates TA, Sampson SD, Delgado de Jesús CR, Zanno LE, Eberth D, Hernandez-Rivera R, Aguillón Martínez MC, Kirkland JI. 2007. Velafrons coahuilensis, a new lambeosaurine hadrosaurid (Dinosauria: Ornithopoda) from the late Campanian Cerro del Pueblo Formation, Coahuila, Mexico. Journal of Vertebrate Paleontology 27: 917-930.

Gauthier J. 1986. Saurischian monophyly and the origin of birds. In Padian K. (ed.). The origin of birds and the evolution of flight. California Academy of Science, San Francisco: 1-55.

Gauthier J, de Queiroz K, Estes R 1988. A phylogenetic analysis of Lepidosauromorpha. In Estes R, Pregill GK. (eds). Phylogenetic Relationships of the Lizard Families: Essays Commemorating Charles L. Camp. Stanford University Press, Stanford, California: 15-98. Gauthier JA, Langer MC, Novas FE, Bittencourt J, Ezcurra MS. 2020. Saurischia. In de Queiroz K, Cantino PD, Gauthier JA (eds). Phylonyms. A Companion to the PhyloCode. CRC Press: $1219-1224$.

Ghiselin MT. 1984. "Definition," "character," and other equivocal terms. Sytematic Zoology 33: $104-110$.

Gilmore CW. 1913. A new dinosaur from the Lance Formation of Wyoming. Smithsonian Miscellaneous Publications 61: 1-5.

Gilmore CW. 1931. A new species of troödont dinosaur from the Lance Formation of Wyoming. Proceedings of the United States National Museum 79: 1-6.

Gilmore CW. 1933. Two new dinosaurian reptiles from Mongolia with notes on some fragmentary specimens. American Museum Novitates 679: 1-20. 
2889 Glut DF. 1997. Dinosaurs: The Encyclopedia, Jefferson, North Carolina, McFarland \& Co.: 1076 2890 p.

2891 Godefroit P, Bolotsky YL, Bolotsky IY. 2012. Osteology and relationships of Olorotitan 2892 arharensis, a hollow-crested hadrosaurid dinosaur from the latest Cretaceous of Far 2893 2894 2895

Granger W, Gregory WK. 1923. Protoceratops andrewsi, a pre-ceratopsian dinosaur from Mongolia, with an appendix on the structural relationships of the Protoceratops beds. American Museum Novitates 72: 1-9.

Han F-L, Barrett PM, Butler RJ, Xu X. 2012. Postcranial anatomy of Jeholosaurus shangyuanensis (Dinosauria, Ornithischia) from the Lower Cretaceous Yixian Formation of China. Journal of Vertebrate Paleontology 32: 1370-1395.

Han F, Forster CA, Xu X, Clark JM. 2018. Postcranial anatomy of Yinlong downsi (Dinosauria: Ceratopsia) from the Upper Jurassic Shishugou Formation of China and the phylogeny of basal ornithischians. Journal of Systematic Palaeontology 16: 1159-1187.

Han F, Forster CA, Clark JM, Xu X. 2015. A New Taxon of Basal Ceratopsian from China and the Early Evolution of Ceratopsia. PLoS ONE 10: e0143369.

Hay OP. 1902. Bibliography and Catalogue of the Fossil Vertebrata of North America. Bulletin of the United States Geological Survey 179: 1-868.

Hedrick BP, Dodson P. 2013. Lujiatun Psittacosaurids: Understanding Individual and Taphonomic Variation Using 3D Geometric Morphometrics. PLoS ONE 8: e69265.

Herne MC, Nair JP, Evans AR, Tait AM. 2019. New small-bodied ornithopods (Dinosauria, Neornithischia) from the Early Cretaceous Wonthaggi Formation (Strzelecki Group) of the Australian-Antarctic rift system, with revision of Qantassaurus intrepidus Rich and Vickers-Rich, 1999. Journal of Paleontology 93: 543-584.

Hill RV, Witmer LW, Norell MA. 2003. A new specimen of Pinacosaurus grangeri (Dinosauria: Ornithischia) from the Late Cretaceous of Mongolia: ontogeny and phylogeny of ankylosaurs. American Museum Novitates 3395: 1-29.

Holmes RB, Persons WS, Rupal BS, Qureshi AJ, Currie PJ. 2020. Morphological variation and asymmetrical development in the skull of Styracosaurus albertensis. Cretaceous Research 107: 104308. 
2919 Horner JR. 1992. Cranial morphology of Prosaurolophus (Ornithischia: Hadrosauridae) with

2920

2921

2922

2923

2924

2925

2926

2927

2928

2929

2930

2931

2932

2933

2934

2935

2936

2937

2938

2939

2940

2941

2942

2943

2944

2945

2946

2947

2948

2949

descriptions of two new hadrosaurid species and an evaluation of hadrosaurid phylogenetic relationships. Museum of the Rockies Occasional Paper 2: 1-119.

Horner JR, Goodwin MB. 2009. Extreme Cranial Ontogeny in the Upper Cretaceous Dinosaur Pachycephalosaurus. PLoS ONE 4: e7626.

Horner JR, Weishampel DB. 1988. A comparative embryological study of two ornithischian dinosaurs. Nature 332: 256-257.

von Huene F. 1950. Bemerkungen zu einem fremdartigen neuen Ceratopsiden. Neues Jahrbuch für Geologie und Paläontologie Monatshefte 1950: 347-351.

von Huene F. 1952. Die Saurierwelt und ihre geschichtlichen Zusammenhänge. Gustav Fischer, Jena, $64 \mathrm{pp}$.

Huxley TH. 1869. On Hypsilophodon foxii, a new genus of Dinosauria. Geological Society of London, Abstracts of Proceedings 204: 3-4.

International Commission on Zoological Nomenclature [ICZN]. 1999. International Code of Zoological Nomenclature. Fourth Edition. The International Trust for Zoological Nomenclature, London, UK. 306 pp.

International Commission on Zoological Nomenclature [ICZN]. 2000. Opinion 1947 Iguanodon Mantell, 1825 (Reptilia: Ornithischia): Iguanodon bernissartensis Boulenger in Beneden, 1881 designated as the type species, and a lectotype designated. Bulletin of Zoological Nomenclature 57: 61-62.

International Commission on Zoological Nomenclature [ICZN]. 2013. Opinion 2320 (Case 3536): Stegosaurus Marsh, 1877 (Dinosauria, Ornithischia): type species replaced with Stegosaurus stenops Marsh, 1887. Bulletin of Zoological Nomenclature 70: 129-130. Jaekel O. 1910. Uber das System der Reptillen. Zeitshrift fur Induktive Abstammungs-und Vererbungslehre 4: 324-341.

Kirkland JI. 1998. A polacanthine ankylosaur (Ornithischia: Dinosauria) from the Early Cretaceous (Barremian) of eastern Utah. New Mexico Museum of Natural History and Science Bulletin 14: 271-281.

Kirkland JI, Alcalá L, Loewen MA, Espílez E, Mampel L, Wiersma JP. 2013. The Basal Nodosaurid Ankylosaur Europelta carbonensis n. gen., n. sp. from the Lower Cretaceous (Lower Albian) Escucha Formation of Northeastern Spain. PLoS ONE 8: e80405.

Peerj reviewing PDF | (2021:04:60693:1:1:NEW 8 Sep 2021) 
2950 Kirkland JI, Carpenter K. 1994. North America’s first pre-Cretaceous ankylosaur (Dinosauria)

2951

2952

2953

2954

2955

2956

2957

2958

2959

2960

2961

2962

2963

2964

2965

2966

2967

2968

2969

2970

2971

2972

2973

2974

2975

2976

2977

2978

2979 from the Upper Jurassic Morrison Formation of western Colorado. Brigham Young University Geology Studies 40: 25-42.

Knapp A, Knell RJ, Farke AA, Loewen MA, Hone DWE. 2018. Patterns of divergence in the morphology of ceratopsian dinosaurs: sympatry is not a driver of ornament evolution. Proceedings of the Royal Society B: Biological Sciences 285: 20180312.

Kobayashi Y, Nishimura T, Takasaki R, Chiba K, Fiorillo AR, Tanaka K, Tsogtbaatar C, Sato T, Sakurai K. 2019. A New Hadrosaurine (Dinosauria: Hadrosauridae) from the Marine Deposits of the Late Cretaceous Hakobuchi Formation, Yezo Group, Japan. Scientific Reports 9: 12389.

Kobayashi Y, Takasaki R, Kubota K, Fiorillo AR. 2021. A new basal hadrosaurid (Dinosauria: Ornithischia) from the latest Cretaceous Kita-ama Formation in Japan implies the origin of hadrosaurids. Scientific Reports 11: 8547.

Kuhn O. 1966. Die Reptilien. Verlag Oeben, Krailling near Munich: 154 pp.

Lambe LM. 1902. New genera and species from the Belly River Series (mid-Cretaceous). Geological Survey of Canada Contributions to Canadian Palaeontology 3: 25-81.

Lambe LM. 1905. On the squamoso-parietal crest of the horned dinosaurs Centrosaurus apertus and Monoclonius canadensis from the Cretaceous of Alberta. Proceedings and Transactions of the Royal Society of Canada, series 2 10:1-9.

Lambe LM. 1915. On Eoceratops canadensis, gen. nov., with remarks on other genera of Cretaceous horned dinosaurs. Canada Geological Survey Museum Bulletin 12, Geological Series 24: 1-49.

Lambe LM. 1917. A new genus and species of crestless hadrosaur from the Edmonton Formation of Alberta. The Ottawa Naturalist 31: 65-73.

Lambe LM. 1918. On the genus Trachodon of Leidy. Ottawa Naturalist 31: 135-139.

Lambe LM. 1919. Description of a new genus and species (Panoplosaurus mirus) of an armoured dinosaur from the Belly River Beds of Alberta. Proceedings and Transactions of the Royal Society of Canada, series 3 13: 39-50.

Langer MC, Ezcurra MD, Bittencourt JS, Novas FE. 2010. The origin and early evolution of dinosaurs. Biological Reviews 85: 55-110.

Peer] reviewing PDF | (2021:04:60693:1:1:NEW 8 Sep 2021) 
2980 Langer MC, Ezcurra MD, Rauhut OWM, Benton MJ, Knoll F, McPhee BW, Novas FE, Pol D,

2981

2982

2983

2984

2985

2986

2987

2988

2989

2990

2991

2992

2993

2994

2995

2996

2997

2998

2999

3000

3001

3002

3003

3004

3005

3006

3007

3008

3009
Brusatte SL. 2017. Untangling the dinosaur family tree. Nature 551: E1-E3.

Langer MC, Ferigolo J. 2013. The Late Triassic dinosauromorph Sacisaurus agudoensis

(Caturrita Formation; Rio Grande do Sul, Brazil): anatomy and affinities. Geological

Society, London, Special Publications 379: 353-392.

Langer MC, Novas FE, Bittencourt JS, Ezcurra MD, Gauthier JA. 2020. Dinosauria. In de

Queiroz K, Cantino PD, Gauthier JA (eds). Phylonyms. A Companion to the PhyloCode. CRC Press: 1209-1217.

Lapparent AF, Lavocat R. 1955. Dinosauriens. In Piveteau J. (ed.). Traité de Paléontologie, Tome 5. Massonet Cie, Paris: 785-962.

Leidy J. 1858. Hadrosaurus foulkii, a new saurian from the Cretaceous of New Jersey, related to Iguanodon. Proceedings of the Academy of Natural Sciences of Philadelphia 10: 213-218.

Longrich NR. 2011. Titanoceratops ouranos, a giant horned dinosaur from the late Campanian of New Mexico. Cretaceous Research 32: 264-276.

Longrich NR, Sankey J, Tanke D. 2010. Texacephale langstoni, a new genus of pachycephalosaurid (Dinosauria: Ornithischia) from the upper Campanian Aguja

Formation, southern Texas, USA. Cretaceous Research 31: 274-284.

Longrich NR, Suberbiola XP, Pyron RA, Jalil N-E. 2021. The first duckbill dinosaur

(Hadrosauridae: Lambeosaurinae) from Africa and the role of oceanic dispersal in dinosaur biogeography. Cretaceous Research 120: 104678.

Lucas SG, Sullivan RM, Lichtig AJ, Dalman AG, Jasinski SE. 2016. Late Cretaceous dinosaur biogeography and endemism in the Western Interior Basin, North America: A critical reevaluation. New Mexico Museum of Natural History and Science Bulletin 71: 195-213.

Lydekker R. 1889. Notes on new and other dinosaurian remains. Geological Magazine, decade 3 6: 352-356.

Madzia D, Boyd CA, Mazuch M. 2018. A basal ornithopod dinosaur from the Cenomanian of the Czech Republic. Journal of Systematic Palaeontology 16: 967-979.

Madzia D, Jagt JWM, Mulder EWA. 2020. Osteology, phylogenetic affinities and taxonomic status of the enigmatic late Maastrichtian ornithopod taxon Orthomerus dolloi (Dinosauria, Ornithischia). Cretaceous Research 108: 104334.

Peer) reviewing PDF | (2021:04:60693:1:1:NEW 8 Sep 2021) 
3010 Maidment SCR, Norman DB, Barrett PM, Upchurch P. 2008. Systematics and phylogeny of

3011

3012

3013

3014

3015

3016

3017

3018

3019

3020

3021

3022

3023

3024

3025

3026

3027

3028

3029

3030

3031

3032

3033

3034

3035

3036

3037

3038

3039

Stegosauria (Dinosauria: Ornithischia). Journal of Systematic Palaeontology 6: 367-407.

Maidment SCR, Raven TJ, Ouarhache D, Barrett PM. 2020. North Africa's first stegosaur:

implications for Gondwanan thyreophoran dinosaur diversity. Gondwana Research 77: 8297.

Makovicky PJ. 2001. A Montanoceratops cerorhynchus (Dinosauria: Ceratopsia) braincase from the Horseshoe Canyon Formation of Alberta. In Tanke D, Carpenter K. (eds). Mesozoic vertebrate life. Bloomington: University of Indiana Press: 243-262.

Mallon JC, Ott CJ, Larson PL, Iuliano EM, Evans DC. 2016. Spiclypeus shipporum gen. et sp. nov., a Boldly Audacious New Chasmosaurine Ceratopsid (Dinosauria: Ornithischia) from the Judith River Formation (Upper Cretaceous: Campanian) of Montana, USA. PLoS ONE 11: $\mathrm{e} 0154218$.

Mantell GA. 1825. Notice on the Iguanodon, a newly discovered fossil reptile, from the sandstone of Tilgate Forest, in Sussex. Philosophical Transactions of the Royal Society of London 115: 179-186.

Mantell GA. 1833. The Geology of the South-East of England: xix-415.

Marsh OC. 1877a. Notice of new dinosaurian reptiles from the Jurassic formation. American Journal of Science and Arts 14: 514-516.

Marsh OC. 1877b. A new order of extinct Reptilia (Stegosauria) from the Jurassic of the Rocky Mountains. American Journal of Science and Arts 14: 513-514.

Marsh OC. 1878. Principal characters of American Jurassic dinosaurs. Part I. American Journal of Science and Arts 16: 411-416.

Marsh OC. 1879. Notice of new Jurassic reptiles. American Journal of Science and Arts 18: 501505.

Marsh OC. 1880. Principal characters of American Jurassic dinosaurs. Part III. American Journal of Science 19: 253-259.

Marsh OC. 1881. Principal characters of American Jurassic dinosaurs. Part V. The American Journal of Science and Arts, series 3 21: 417-423.

Marsh OC. 1882. Classification of the Dinosauria. American Journal of Science 23: 81-86.

Marsh OC. 1885. Names of extinct reptiles. American Journal of Science 29: 169.

Peer) reviewing PDF | (2021:04:60693:1:1:NEW 8 Sep 2021) 
3040 Marsh OC. 1887. Principal characters of American Jurassic dinosaurs. Part IX. The skull and 3041 dermal armor of Stegosaurus. American Journal of Science 34: 413-417.

3042 Marsh OC. 1888. A new family of horned Dinosauria, from the Cretaceous. The American $3043 \quad$ Journal of Science, series 3 36: 477-478.

3044 Marsh OC. 1889. Notice of gigantic horned Dinosauria from the Cretaceous. American Journal 3045 of Science 38: 173-175.

3046 Marsh OC. 1890. Additional characters of the Ceratopsidae, with notice of new Cretaceous 3047 dinosaurs. American Journal of Science 39: 418-426.

3048 Marsh OC. 1894. The typical Ornithopoda of the American Jurassic. American Journal of 3049 Science, series 3 48: 85-90.

3050

3051

3052

3053

3054

3055

3056

3057

3058

3059

3060

3061

3062

3063

3064

3065

3066

3067

Martz JW, Small BJ. 2019. Non-dinosaurian dinosauromorphs from the Chinle Formation (Upper Triassic) of the Eagle Basin, northern Colorado: Dromomeron romeri (Lagerpetidae) and a new taxon, Kwanasaurus williamparkeri (Silesauridae). PeerJ 7: e7551.

Maryańska T. 1977. Ankylosauridae (Dinosauria) from Mongolia. Palaeontologia Polonica 37: 85-151.

Maryańska T, Chapman RE, Weishampel DB. 2004. Pachycephalosauria. In Weishampel DB, Dodson P, Osmólska H. (eds). The Dinosauria. 2nd Ed. University of California Press, Berkeley: 464-477.

Maryańska T, Osmólska H. 1974. Pachycephalosauria, a new suborder of ornithischian dinosaurs. Palaeontologia Polonica 30: 45-102.

Matheron P. 1869. Notice sur les reptiles fossiles des dépôts fluvio-lacustres crétacés du bassin à lignite de Fuveau. Mémoires de l'Académie des Sciences, Belles-Lettres, et Arts de Marseille 1868-1869: 345-379.

McDonald AT. 2011. The taxonomy of species assigned to Camptosaurus (Dinosauria: Ornithopoda). Zootaxa 2783: 52-68.

McDonald AT, Wolfe DG, Freedman Fowler EA, Gates TA. 2021. A new brachylophosaurin (Dinosauria: Hadrosauridae) from the Upper Cretaceous Menefee Formation of New 3068 Mexico. PeerJ 9: e11084. 
3069 McFeeters BD, Evans DC, Ryan MJ, Maddin HC. 2021a. First occurrence of Maiasaura 3070 (Dinosauria, Hadrosauridae) from the Upper Cretaceous Oldman Formation of southern Alberta, Canada. Canadian Journal of Earth Sciences 58: 286-296.

3072

3073

3074

3075

3076

3077

3078

3079

3080

3081

3082

3083

3084

3085

3086

3087

3088

3089

3090

3091

3092

3093

3094

3095

3096

3097

3098

3099

McFeeters B, Evans DC, Maddin HC. 2021b. Ontogeny and variation in the skull roof and braincase of the hadrosaurid dinosaur Maiasaura peeblesorum from the Upper Cretaceous of Montana, USA. Acta Palaeontologica Polonica 66. doi: 10.4202/app.00698.2019

Milner AR, Norman DB. 1984. The biogeography of advanced ornithopod dinosaurs (Archosauria: Ornithischia) — a cladistic-vicariance model. In Reif WE, Westphal F. (eds.). Third Symposium on Mesozoic Terrestrial Ecosystems, Short Papers. Attempto Verlag, Tübingen: $145-150$.

Morschhauser EM, You H, Li D, Dodson P. 2019. Phylogenetic history of Auroraceratops rugosus (Ceratopsia: Ornithischia) from the Lower Cretaceous of Gansu Province, China: 117-147. In You H, Dodson P, Morschhauser E. Auroraceratops rugosus (Ornithischia, Ceratopsia) from the Early Cretaceous of northwestern Gansu Province, China. Society of Vertebrate Paleontology Memoir 18. Journal of Vertebrate Paleontology 38 (Supplement).

Müller RT, Dias-da-Silva S. 2019. Taxon sample and character coding deeply impact unstable branches in phylogenetic trees of dinosaurs. Historical Biology 31: 1089-1092.

Müller RT, Garcia MS. 2020. A paraphyletic 'Silesauridae' as an alternative hypothesis for the initial radiation of ornithischian dinosaurs. Biology Letters 16: 20200417.

Müller RT, Pretto FA, Kerber L, Silva-Neves E, Dias-da-Silva S. 2018. Comment on 'A dinosaur missing-link? Chilesaurus and the early evolution of ornithischian dinosaurs'. Biology Letters 14: 20170581.

Naish D, Cau A, Holtz, Jr. TR, Fabbri M, Gauthier JA. 2020. Theropoda. In de Queiroz K, Cantino PD, Gauthier JA (eds). Phylonyms. A Companion to the PhyloCode. CRC Press: $1235-1246$.

Nesbitt SJ, Sidor CA, Irmis RB, Angielczyk KD, Smith RMH, Tsuji LA. 2010. Ecologically distinct dinosaurian sister group shows early diversification of Ornithodira. Nature 464: 95-98.

Nopcsa F. 1900. Dinosaurierreste aus Siebenbürgen (Schädel von Limnosaurus transsylvanicus nov. gen. et spec.). Denkschriften der Kaiserlichen Akademie der Wissenschaften. Mathematisch-Naturwissenschaftliche Classe 68: 555-591. 
3100 Nopcsa F. 1915. Die dinosaurier der Siebenbürgischen landesteile Ungarns. Mitteilungen aus den

3101

3102

3103

3104

3105

3106

3107

3108

3109

3110

3111

3112

3113

3114

3115

3116

3117

3118

3119

3120

3121

3122

3123

3124

3125

3126

3127

3128 Jahrbuch der Königlich Ungarnischen Geologischen Reichsanstalt 23: 1-24.

Nopcsa F. 1918. Leipsanosaurus n. gen. In neuer Thyreophoreaus der Gosau. Foldtani Kozlony 48: $324-328$.

Nopcsa F. 1923. Die Familien der Reptilien. Forschritte der Geologie und Palaeontologie. Verlag von Gebrüder Borntraeger, Berlin 2: 1-210.

Nopcsa F. 1929. Dinosaurierreste aus Siebenbürgen V. Geologica Hungarica, Series Palaeontologica 4: 1-72.

Norman DB. 1980. On the ornithischian dinosaur Iguanodon bernissartensis from the Lower Cretaceous of Bernissart (Belgium). Memoires de 1'Institut Royal des Sciences Naturelles de Belgique 178: 1-103.

Norman DB. 2002. On Asian ornithopods (Dinosauria: Ornithischia). 4. Redescription of Probactrosaurus gobiensis Rozhdestvensky 1966. Zoological Journal of the Linnean Society 136: 113-144.

Norman DB. 2014. Iguanodonts from the Wealden of England: do they contribute to the discussion concerning hadrosaur origins? In Eberth DA, Evans DC. (eds). Hadrosaurs, Indiana University Press, Bloomington: 10-43.

Norman DB. 2015. On the history, osteology, and systematic position of the Wealden (Hastings group) dinosaur Hypselospinus fittoni (Iguanodontia: Styracosterna) Zoological Journal of the Linnean Society 173: 92-189.

Norman DB. 2020a. Scelidosaurus harrisonii from the Early Jurassic of Dorset, England: cranial anatomy. Zoological Journal of the Linnean Society 188: 1-81.

Norman DB. 2020b. Scelidosaurus harrisonii from the Early Jurassic of Dorset, England: postcranial skeleton. Zoological Journal of the Linnean Society 189: 47-157.

Norman DB. 2020c. Scelidosaurus harrisonii from the Early Jurassic of Dorset, England: the dermal skeleton. Zoological Journal of the Linnean Society 190: 1-53.

Norman DB. 2021. Scelidosaurus harrisonii (Dinosauria: Ornithischia) from the Early Jurassic of Dorset, England: biology and phylogenetic relationships. Zoological Journal of the Linnean Society 191: 1-86. 
3129 Norman DB, Butler RJ, Maidment SCR. 2007. Reconsidering the status and affinities of the 3130 ornithischian dinosaur Tatisaurus oehleri Simmons, 1965. Zoological Journal of the $3131 \quad$ Linnean Society 150: 865-874.

3132 Norman DB, Witmer LM, Weishampel DB. 2004a. Basal Ornithischia. In Weishampel DB, 3133 Dodson P. Osmólska H. (eds), The Dinosauria. 2nd Ed. University of California Press, 3134 Berkeley: 325-334.

3135 Norman DB, Sues H-D, Witmer LM, Coria RA. 2004b. Basal Ornithopoda. In Weishampel DB, 3136 Dodson P, Osmólska H. (eds). The Dinosauria. 2nd Ed. University of California Press, 3137 Berkeley: 393-412.

3138 Novas FE, Cambiaso AV, Ambrosio A. 2004. A new basal iguanodontian (Dinosauria, 3139 Ornithischia) from the Upper Cretaceous of Patagonia. Ameghiniana 41: 75-82.

3140 Novas FE, Salgado L, Suárez M, Agnolín FL, Ezcurra MD, Chimento NR, de la Cruz R, Isasi MP, Vargas AO, Rubilar-Rogers D. 2015. An enigmatic plant-eating theropod from the Late Jurassic period of Chile. Nature 522: 331-334.

Osborn HF. 1923. Two Lower Cretaceous dinosaurs of Mongolia. American Museum Novitates 95: $1-10$.

Ostrom JH. 1970. Stratigraphy and paleontology of the Cloverly Formation (Lower Cretaceous) of the Bighorn Basin area, Wyoming and Montana. Peabody Museum Bulletin 35: 1-234. Ösi A. 2015. The European ankylosaur record: a review. Hantkeniana 10: 89-106.

Pacheco C, Müller RT, Langer M, Pretto FA, Kerber L, Dias da Silva S. 2019. Gnathovorax cabreirai: a new early dinosaur and the origin and initial radiation of predatory dinosaurs. PeerJ 7: e7963.

Padian K, May CL. 1993. The earliest dinosaurs. In Lucas SG, Morales M. (eds). The Nonmarine Triassic. New Mexico Museum of Natural History and Science, Albuquerque: 379-381.

Parks WA. 1922. Parasaurolophus walkeri, a new genus and species of crested trachodont dinosaur. University of Toronto Studies, Geology Series 13:1-32.

Parks WA. 1923. Corythosaurus intermedius, a new species of trachodont dinosaur. University of Toronto Studies, Geological Series 15: 1-57.

Parks WA. 1925. Arrhinoceratops brachyops, a new genus and species of Ceratopsia from the Edmonton Formation of Alberta. University of Toronto Studies, Geology Series 19: 1-15. 
3160 Párraga J, Prieto-Márquez A. 2019. Pareisactus evrostos, a new basal iguanodontian

3161 (Dinosauria: Ornithopoda) from the Upper Cretaceous of southwestern Europe. Zootaxa $3162 \quad 4555: 247-258$

3163 Peecook BR, Sidor CA, Nesbitt SJ, Smith RMH, Steyer JS, Angielczyk KD. 2013. A new

3164 silesaurid from the upper Ntawere Formation of Zambia (Middle Triassic) demonstrates

3165 the rapid diversification of Silesauridae (Avemetatarsalia, Dinosauriformes). Journal of

$3166 \quad$ Vertebrate Paleontology 33: 1127-1137.

3167 Pereda-Suberbiola X, Canudo JI, Cruzado-Caballero P, Barco JL, López-Martínez N, Oms O, 3168 Ruiz-Omeñaca JI. 2009. The last hadrosaurid dinosaurs of Europe: a new lambeosaurine 3169 from the uppermost Cretaceous of Aren (Huesca, Spain). Comptes Rendus Palevol 8: 559$3170 \quad 572$.

3171 Prieto-Márquez A. 2010. Global phylogeny of Hadrosauridae (Dinosauria: Ornithopoda) using 3172 parsimony and Bayesian methods. Zoological Journal of the Linnean Society 159: 4353173502.

3174 Prieto-Márquez A. 2014. Skeletal morphology of Kritosaurus navajovius (Dinosauria:

3175 Hadrosauridae) from the Late Cretaceous of the North American south-west, with an 3176 evaluation of the phylogenetic systematics and biogeography of Kritosaurini. Journal of 3177 Systematic Palaeontology 12: 133-175.

3178 Prieto-Márquez A, Dalla Vecchia FM, Gaete R, Galobart À. 2013. Diversity, Relationships, and 3179 Biogeography of the Lambeosaurine Dinosaurs from the European Archipelago, with 3180 Description of the New Aralosaurin Canardia garonnensis. PLoS ONE 8: e69835.

3181 Prieto-Márquez A, Erickson GM, Ebersole JA. 2016. A primitive hadrosaurid from southeastern 3182 North America and the origin and early evolution of 'duck-billed' dinosaurs. Journal of 3183 Vertebrate Paleontology 36: e1054495.

3184 Prieto-Márquez A, Fondevilla V, Sellés AG, Wagner JR, Galobart À. 2019. Adynomosaurus 3185 arcanus, a new lambeosaurine dinosaur from the Late Cretaceous Ibero-Armorican Island 3186 of the European Archipelago. Cretaceous Research 96: 19-37.

3187 Prieto-Márquez A, Wagner JR, Bell PR, Chiappe LM. 2014. The late-surviving 'duck-billed' 3188 dinosaur Augustynolophus from the upper Maastrichtian of western North America and 3189 crest evolution in Saurolophini. Geological Magazine 152: 225-241. 
3190 Prieto-Márquez A, Wagner JR, Lehman T. 2020. An unusual 'shovel-billed' dinosaur with 3191 trophic specializations from the early Campanian of Trans-Pecos Texas, and the ancestral 3192 hadrosaurian crest. Journal of Systematic Palaeontology 18: 461-498.

3193 Ramírez-Velasco ÁA, Aguilar FJ, Hernández-Rivera R, Gudiño Maussán JL, Rodriguez ML, Alvarado-Ortega J. 2021. Tlatolophus galorum, gen. et sp. nov., a parasaurolophini dinosaur from the upper Campanian of the Cerro del Pueblo Formation, Coahuila, northern Mexico. Cretaceous Research: 104884.

Raven TJ, Barrett PM, Xu X, Maidment SCR. 2019. A reassessment of the purported ankylosaurian dinosaur Bienosaurus lufengensis from the Lower Lufeng Formation of

Raven TJ, Maidment SCR. 2017. A new phylogeny of Stegosauria (Dinosauria, Ornithischia). Palaeontology 60: 401-408.

Rivera-Sylva HE, Frey E, Stinnesbeck W, Carbot-Shanona G, Sanchez-Uribe IE, GuzmánGutiérrez JR. 2018a. Paleodiversity of Late Cretaceous Ankylosauria from Mexico and their phylogenetic significance. Swiss Journal of Palaeontology 137: 83-93.

Rivera-Sylva HE, Frey E, Stinnesbeck W, Amezcua N, Flores Huerta D. 2018b. First occurrence of Parksosauridae in Mexico, from the Cerro del Pueblo Formation (Late Cretaceous; late Campanian) at Las Águilas, Coahuila: Boletín de la Sociedad Geológica Mexicana 70: 779-784.

Romer AS. 1966. Vertebrate Paleontology, 3rd edition, 468 pp.

Rotatori FM, Moreno-Azanza M, Mateus O. 2020. New information on ornithopod dinosaurs from the Late Jurassic of Portugal. Acta Palaeontologica Polonica 65: 35-57.

Rowe T. 1987. Definition and diagnosis in the phylogenetic system. Systematic Zoology 36: 208-211.

Rozadilla S, Agnolín FL, Novas FE. Osteology of the Patagonian ornithopod Talenkauen santacrucensis (Dinosauria, Ornithischia). Journal of Systematic Palaeontology 17: 20432089.

Rozhdestvensky AK. 1966. Novye igyanodonti ie tsentrallinoy Asii. Phillogeneticheskye y taksonomicheskye veaimoothoshenia poednich Iguanodontidae y rannich Hadrosauridae. Paleontologicheskii Zhurnal 1966: 103-116. 
3220 Rozhdestvensky AK. 1968. Gadrozavry Kazakhstana. In Tatarinov LP et al. (ed.). Akademia $3221 \quad$ Naul SSSR, Moscow: 97-141.

3222 Ryan MJ, Holmes R, Mallon J, Loewen M, Evans DC. 2017. A basal ceratopsid (Centrosaurinae: Nasutoceratopsini) from the Oldman Formation (Campanian) of Alberta, Canada. Canadian Journal of Earth Sciences 54: 1-14.

Sampson SD. 1995. Two new horned dinosaurs from the upper Cretaceous Two Medicine Formation of Montana; with a phylogenetic analysis of the Centrosaurinae (Ornithischia: Ceratopsidae). Journal of Vertebrate Paleontology 15: 743-760.

Sampson SD, Lund EK, Loewen MA, Farke AA, Clayton KE. 2013. A remarkable short-snouted horned dinosaur from the Late Cretaceous (late Campanian) of southern Laramidia. Proceedings of the Royal Society B: Biological Sciences 280: 20131186.

Santos-Cubedo A, de Santisteban C, Poza B, Meseguer S. 2021. A new styracosternan hadrosauroid (Dinosauria: Ornithischia) from the Early Cretaceous of Portell, Spain. PLoS ONE 16: e0253599.

Schott RK, Evans DC. 2017. Cranial variation and systematics of Foraminacephale brevis gen. nov. and the diversity of pachycephalosaurid dinosaurs (Ornithischia: Cerapoda) in the Belly River Group of Alberta, Canada. Zoological Journal of the Linnean Society 179: 865-906.

Seeley HG. 1888. On the classification of the fossil animals commonly named Dinosauria. Proceedings of the Royal Society of London 43: 165-171.

Sereno PC. 1986. Phylogeny of the bird-hipped dinosaurs. National Geographic Research 2: $234-256$.

Sereno PC. 1997. The origin and evolution of dinosaurs. Annual Review of Earth and Planetary Sciences 25: 435-489.

Sereno PC. 1998. A rationale for phylogenetic definitions, with application to the higher level taxonomy of Dinosauria. Neues Jahrbuch für Geologie und Paläontologie Abhandlungen 210: 41-83.

Sereno PC. 1999. The evolution of dinosaurs. Science 284: 2137-2147.

Sereno PC. 2005. Stem Archosauria-TaxonSearch. http://taxonsearch.uchicago.edu/ [accessed May $\left.4^{\text {th }}, 2021\right]$ 
3250 Sereno PC. 2012. Taxonomy, morphology, masticatory function and phylogeny of

3251 heterodontosaurid dinosaurs. ZooKeys 226: 1-225.

3252 Słowiak J, Szczygielski T, Ginter M, Fostowicz-Frelik Ł. 2020. Uninterrupted growth in a non-polar hadrosaur explains the gigantism among duck-billed dinosaurs. Palaeontology 63: 579-599.

Sternberg CM. 1937. Classification of Thescelosaurus, with a description of a new species. Proceedings of the Geological Society of America 1936: 375.

Sternberg CM. 1940. Thescelosaurus edmontonensis, n. sp., and classification of the Hypsilophodontidae. Journal of Paleontology 14: 481-494.

Sternberg CM. 1945. Pachycephalosauridae proposed for dome-headed dinosaurs, Stegoceras lambei, n. sp., described. Journal of Paleontology 19: 534-538.

Sternberg CM. 1950. Pachyrhinosaurus canadensis, representing a new family of the Ceratopsia, from southern Alberta. National Museum of Canada Bulletin 118: 109-120.

Sternberg CM. 1953. A new hadrosaur from the Oldman Formation of Alberta: discussion of nomenclature. National Museum of Canada Bulletin 128: 1-12.

Stubbs TL, Benton MJ, Elsler A, Prieto-Márquez A. 2019. Morphological innovation and the evolution of hadrosaurid dinosaurs. Paleobiology 45: 347-362.

Sullivan RM. 2003. Revision of the dinosaur Stegoceras Lambe (Ornithischia, Pachycephalosauridae). Journal of Vertebrate Paleontology 23: 181-207.

Sullivan RM, Jasinski SE, Guenther M, Lucas SG. 2011. The first lambeosaurin (Dinosauria, Hadrosauridae, Lambeosaurinae) from the Upper Cretaceous Ojo Alamo Formation (Naashoibito Member), San Juan Basin, New Mexico. In Sullivan RM, Lucas SG, Spielmann JA. (eds.). Fossil Record 3. New Mexico Museum of Natural History and Science Bulletin: 405-417.

Tennant JP, Chiarenza AA, Baron M. 2018. How has our knowledge of dinosaur diversity through geologic time changed through research history? PeerJ 6: e4417.

Thompson RS, Parish JC, Maidment SCR, Barrett PM. 2012. Phylogeny of the ankylosaurian dinosaurs (Ornithischia: Thyreophora). Journal of Systematic Palaeontology 10: 301-312.

Tsogtbaatar K, Weishampel DB, Evans DC, Watabe M. 2019. A new hadrosauroid (Dinosauria: Ornithopoda) from the Late Cretaceous Baynshire Formation of the Gobi Desert (Mongolia). PLoS ONE 14: e0208480. 
3281 Tumanova TA. 1983. Pervyy ankilozavr iz nizhnego mela Mongolii. In Tatarinov LP, Barsbold

3282 R, Vorobyeva E, Luvsandanzan B, Trofimov BA, Reshetov YA, Shishkin MA (eds.).

3283 Iskopayemyye reptilii mongolii. Trudy Sovmestnaya Sovetsko-Mongol'skaya

3284 Paleontologicheskaya Ekspeditsiya 24: 110-118.

3285 Tumanova TA. 1987. Pantsirnyye dinozavry Mongolii. Trudy Sovmestnaya Sovetsko-

$3286 \quad$ Mongol'skaya Paleontologicheskaya Ekspeditsiya 32: 1-80.

3287 Verdú FJ, Godefroit P, Royo-Torres R, Cobos A, Alcalá L. 2017. Individual variation in the 3288 postcranial skeleton of the Early Cretaceous Iguanodon bernissartensis (Dinosauria:

3289 Ornithopoda). Cretaceous Research 74: 65-86.

3290 Verdú FJ, Royo-Torres R, Cobos A, Alcalá L. 2018. New systematic and phylogenetic data 3291 about the early Barremian Iguanodon galvensis (Ornithopoda: Iguanodontoidea) from 3292 Spain. Historical Biology 30: 437-474.

3293 Verdú FJ, Royo-Torres R, Cobos A, Alcalá L. 2020. Systematics and paleobiology of a new articulated axial specimen referred to Iguanodon cf. galvensis (Ornithopoda, Iguanodontoidea). Journal of Vertebrate Paleontology 40: e1878202.

Vickaryous MK, Maryanska T, Weishampel DB. 2004. Ankylosauria. In Weishampel DB, Dodson P, Osmólska H. (eds.). The Dinosauria. 2nd Ed. University of California Press, Berkeley: 363-392.

Vickaryous MK, Russell AP, Currie PJ, Zhao X-J. 2001. A new ankylosaurid (Dinosauria: Ankylosauria) from the Lower Cretaceous of China, with comments on ankylosaurian relationships. Canadian Journal of Earth Sciences 38: 1767-1780.

Villanueva-Amadoz U, Sender LM, Alcalá L, Pons D, Royo-Torres R, Diez JB. 2015. Paleoenvironmental reconstruction of an Albian plant community from the Ariño bonebed layer (Iberian Chain, NE Spain). Historical Biology 27: 430-441.

Weishampel DB. 2004. Ornithischia. In Weishampel DB, Dodson P, Osmólska H. (eds). The Dinosauria. 2nd Ed. University of California Press, Berkeley: 323-324. Noto CR. 2004. Dinosaur Distribution. In Weishampel DB, Dodson P, Osmólska H. (eds). The Dinosauria. 2nd Ed. University of California Press, Berkeley: 517-606. 
3310 Weishampel DB, Jianu C-M, Csiki Z, Norman DB. 2003. Osteology and phylogeny of Zalmoxes

3311

3312

3313

3314

3315

3316

3317

3318

3319

3320

3321

3322

3323

3324

3325

3326

3327

3328

3329

3330

3331

3332

3333

3334

3335

3336

3337

3338

3339

(n. g.), an unusual euornithopod dinosaur from the latest Cretaceous of Romania. Journal of Systematic Palaeontology 1: 65-123.

Weishampel DB, Norman DB, Grigorescu D. 1993. Telmatosaurus transsylvanicus from the Late Cretaceous of Romania: the most basal hadrosaurid dinosaur. Palaeontology 36: 361385.

Wiersma JP, Irmis RB. 2018. A new southern Laramidian ankylosaurid, Akainacephalus johnsoni gen. et sp. nov., from the upper Campanian Kaiparowits Formation of southern Utah, USA. PeerJ 6: e5016.

Williamson TE, Brusatte SL. 2016. Pachycephalosaurs (Dinosauria: Ornithischia) from the Upper Cretaceous (upper Campanian) of New Mexico: a reassessment of Stegoceras novomexicanum. Cretaceous Research 62: 29-43.

Williamson TE, Carr TD. 2003. A new genus of derived pachycephalosaurian from western North America. Journal of Vertebrate Paleontology 22: 779-801.

Wilson JP, Ryan MJ, Evans DC. 2020. A new, transitional centrosaurine ceratopsid from the Upper Cretaceous Two Medicine Formation of Montana and the evolution of the 'Styracosaurus-line' dinosaurs. Royal Society Open Science 7: 200284.

Woodruff DC, Goodwin MB, Lyson TR, Evans DC. 2021. Ontogeny and variation of the pachycephalosaurine dinosaur Sphaerotholus buchholtzae, and its systematics within the genus. Zoological Journal of the Linnean Society. doi: 10.1093/zoolinnean/zlaa179

Xing H, Mallon JC, Currie ML. 2017. Supplementary cranial description of the types of Edmontosaurus regalis (Ornithischia: Hadrosauridae), with comments on the phylogenetics and biogeography of Hadrosaurinae. PLoS ONE 12: e0175253.

Xing H, Zhao X, Wang K, Li D, Chen S, Mallon JC, Zhang Y, Xu X. 2014. Comparative Osteology and Phylogenetic Relationship of Edmontosaurus and Shantungosaurus (Dinosauria: Hadrosauridae) from the Upper Cretaceous of North America and East Asia. Acta Geologica Sinica 88: 1623-1652.

Xu X, Forster CA, Clark JM, Mo J. 2006. A basal ceratopsian with transitional features from the Late Jurassic of northwestern China. Proceedings of the Royal Society B: Biological Sciences 273: 2135-2140. 
3340 Yang J-T, You H-L, Li D-Q, Kong D-L. 2013. First discovery of polacanthine ankylosaur 3341 dinosaur in Asia. Vertebrata PalAsiatica 51: 265-277.

3342 Yang Y, Wu W, Dieudonné P, Godefroit P. 2020. A new basal ornithopod dinosaur from the $3343 \quad$ Lower Cretaceous of China. PeerJ 8: e9832.

3344 You H, Dodson P. 2004. Basal Ceratopsia. In Weishampel DB, Osmólska H, Dodson P. (eds).

3345 The Dinosauria. 2nd Ed. University of California Press, Berkeley: 478-493.

3346 Young C-C. 1958. The dinosaurian remains of Laiyang, Shantung. Palaeontologia Sinica, New 3347 Series C, Whole Number 42: 1-138.

3348 Yu C, Prieto-Marquez A, Chinzorig T, Badamkhatan Z, Norell M. 2020. A neoceratopsian 3349 dinosaur from the early Cretaceous of Mongolia and the early evolution of ceratopsia. $3350 \quad$ Communications Biology 3: 499.

3351 Zhang J, Wang X, Wang Q, Jiang S, Cheng X, Li N, Qiu R. 2019. A new saurolophine 3352 hadrosaurid (Dinosauria: Ornithopoda) from the Upper Cretaceous of Shandong, China. 3353 Anais da Academia Brasileira de Ciências 91: e20160920.

3354 Zhang Y-G, Wang K-B, Chen S-Q, Liu D, Xing H. 2020. Osteological Re-Assessment and 3355 Taxonomic Revision of "Tanius laiyangensis" (Ornithischia: Hadrosauroidea) from the 3356 Upper Cretaceous of Shandong, China. The Anatomical Record 303: 790-800.

3357 Zhao X, Cheng Z, Xu X. 1999. The earliest ceratopsian from the Tuchengzi Formation of 3358 Liaoning, China. Journal of Vertebrate Paleontology 19: 681-691.

3359 Zheng W, Jin X, Azuma Y, Wang Q, Miyata K, Xu X. 2018. The most basal ankylosaurine 3360 dinosaur from the Albian-Cenomanian of China, with implications for the evolution of the $3361 \quad$ tail club. Scientific Reports 8: 3711. 
3363 Figure 1. Specifier-based phylogeny of Ornithischia. Subclade topologies reflect those of the 3364 primary reference phylogenies: Ankylosauria (Figure 11 of Arbour \& Currie, 2016; Figure 5 of 3365 Rivera-Sylva et al., 2018a), Hadrosauridae (Figure 25 of Prieto-Márquez et al., 2013; Figure 18 3366 of Prieto-Márquez et al., 2020), Marginocephalia (Figure 27 of Schott \& Evans, 2017; Figure 9 3367 of Chiba et al., 2018; Figure 9a of Fowler \& Freedman Fowler, 2020; Figure 10 of Morschhauser 3368 et al., 2019; Figure 4 of Yu et al., 2020), non-ankylosaur Thyreophora (Figure 16 of Han et al., 2018), non-cerapod Neornithischia (Figure 4 of Madzia et al., 2018), non-genasaur Ornithischia (Figure 4 of Madzia et al., 2018), non-hadrosaurid Ornithopoda (Figure 2.26 of Norman, 2014; Figure 4 of Madzia et al., 2018; Figure 12 of Madzia et al., 2020). Abbreviations: Ch. Chasmosaurinae; Ni. - Neoiguanodontia; Pd. - Pachycephalosauridae; Pn. Pachycephalosaurinae; Pr. - Pachyrostra; Rh. - Rhabdodontomorpha; Rd. - Rhabdodontidae; and Sh. - Shamosaurinae. Majority of the silhouettes were obtained from phylopic.org: Ankylosaurinae (Andrew A. Farke, CC BY 3.0), Camptosauridae (Tasman Dixon, public domain), Centrosaurinae (Andrew A. Farke, CC BY 3.0), Chaoyangsauridae (Andrew A. Farke, CC BY 3.0), Chasmosaurinae (Jagged Fang Designs, public domain), Dryosauridae (Gereth Monger, CC BY 3.0), Heterodontosauridae (Scott Hartman, CC BY 3.0), Iguanodontidae (Tasman Dixon, public domain), Lambeosaurinae (Dmitry Bogdanov, CC BY 3.0), Nodosaurinae (Scott Hartman, public domain), Polacanthinae (FunkMonk, public domain), Protoceratopsidae (Andrew A. Farke, CC BY 3.0), Rhabdodontidae (Scott Hartman, CC BY 3.0), Stegosauria (Scott Hartman, CC BY 3.0). We have further added silhouettes for Elasmaria (Victoria M. Arbour, CC BY 4.0), Pachycephalosauria (Victoria M. Arbour, CC BY 4.0), Saurolophinae (Victoria M. Arbour, CC BY 4.0), and Thescelosauridae (Victoria M. Arbour, CC BY 4.0).

Figure 2. The phylogenetic nomenclature of ornithischian dinosaurs using the topology of Madzia et al. (2018: Fig. 4B). Note that Nanosaurus agilis has been analyzed by Madzia et al. (2018) as 'Othnielosaurus'. The name was changed here following Carpenter \& Galton (2018). Additionally, the name Marasuchus lilloensis was placed in quotation marks to highlight that the taxon may not be distinct from Lagosuchus talampayensis (Agnolin \& Ezcurra, 2019). 
3393 Figure 3. The phylogenetic nomenclature of ornithischian dinosaurs using the topology of 3394 Dieudonné et al. (2020: Figs 1 and 2). Note that Dieudonné et al. (2020) followed Carpenter \& 3395 Lamanna (2015) in placing aphanoecetes within Camptosaurus. Owing to the results of recent 3396 phylogenetic analyses (e.g., Madzia et al., 2020; Verdú et al., 2020), aphanoecetes is placed here 3397 within Uteodon McDonald, 2011. Additionally, the name Psittacosaurus major was changed to 3398 Psittacosaurus lujiatunensis (following Hedrick \& Dodson, 2013), and Ankylosauria and 3399 Stegosauria of Dieudonné et al. (2020) were placed in quotation marks to highlight that these 3400 names have not been necessarily used by the authors as defined in the present study. Note also 3401 that the extent of Ornithischia is difficult to indicate on the tree because Chilesaurus 3402 diegosuarezi may represent a theropod (see Discussion). Abbreviation: An. - Ankylosauria.

3404 Figure 4. The phylogenetic nomenclature of ornithischian dinosaurs using the topology of Yang 3405 et al. (2020: Fig. 12). Ankylosauria and Stegosauria of Yang et al. (2020) were placed in quotation marks to highlight that these names have not been necessarily used by the authors as defined in the present study. In turn, Psittacosauridae of Yang et al. (2020) was placed in quotation marks because the name has not been formally defined yet. Abbreviation: St. Stegosauria.

3411 Figure 5. Specifier-based phylogeny of Hadrosauridae showing alternative placements of 3412 Hadrosaurus foulkii. The silhouette of Lambeosaurinae was obtained from phylopic.org (Dmitry 3413 Bogdanov, CC BY 3.0). The silhouette of Hadrosaurinae/Saurolophinae was prepared by 3414 Victoria M. Arbour (CC BY 4.0). 


\section{Figure 1}

Specifier-based phylogeny of Ornithischia.

Subclade topologies reflect those of the primary reference phylogenies: Ankylosauria (Figure 11 of Arbour \& Currie, 2016; Figure 5 of Rivera-Sylva et al., 2018a), Hadrosauridae (Figure 25 of Prieto-Márquez et al., 2013; Figure 18 of Prieto-Márquez et al., 2020), Marginocephalia (Figure 27 of Schott \& Evans, 2017; Figure 9 of Chiba et al., 2018; Figure 9a of Fowler \& Freedman Fowler, 2020; Figure 10 of Morschhauser et al., 2019; Figure 4 of Yu et al., 2020), non-ankylosaur Thyreophora (Figure 16 of Han et al., 2018), non-cerapod Neornithischia (Figure 4 of Madzia et al., 2018), non-genasaur Ornithischia (Figure 4 of Madzia et al., 2018), non-hadrosaurid Ornithopoda (Figure 2.26 of Norman, 2014; Figure 4 of Madzia et al., 2018; Figure 12 of Madzia et al., 2020). Abbreviations: Ch. - Chasmosaurinae; Ni. Neoiguanodontia; Pd. - Pachycephalosauridae; Pn. - Pachycephalosaurinae; Pr. - Pachyrostra; Rh. - Rhabdodontomorpha; Rd. - Rhabdodontidae; and Sh. - Shamosaurinae. Majority of the silhouettes were obtained from phylopic.org: Ankylosaurinae (Andrew A. Farke, CC BY 3.0), Camptosauridae (Tasman Dixon, public domain), Centrosaurinae (Andrew A. Farke, CC BY 3.0), Chaoyangsauridae (Andrew A. Farke, CC BY 3.0), Chasmosaurinae (Jagged Fang Designs, public domain), Dryosauridae (Gereth Monger, CC BY 3.0), Heterodontosauridae (Scott Hartman, CC BY 3.0), Iguanodontidae (Tasman Dixon, public domain), Lambeosaurinae (Dmitry Bogdanov, CC BY 3.0), Nodosaurinae (Scott Hartman, public domain), Polacanthinae (FunkMonk, public domain), Protoceratopsidae (Andrew A. Farke, CC BY 3.0), Rhabdodontidae (Scott Hartman, CC BY 3.0), Stegosauria (Scott Hartman, CC BY 3.0). We have further added silhouettes for Elasmaria (Victoria M. Arbour, CC BY 4.0), Pachycephalosauria (Victoria M. Arbour, CC BY 4.0), Saurolophinae (Victoria M. Arbour, CC BY 4.0), and Thescelosauridae (Victoria M. Arbour, CC BY 4.0). 


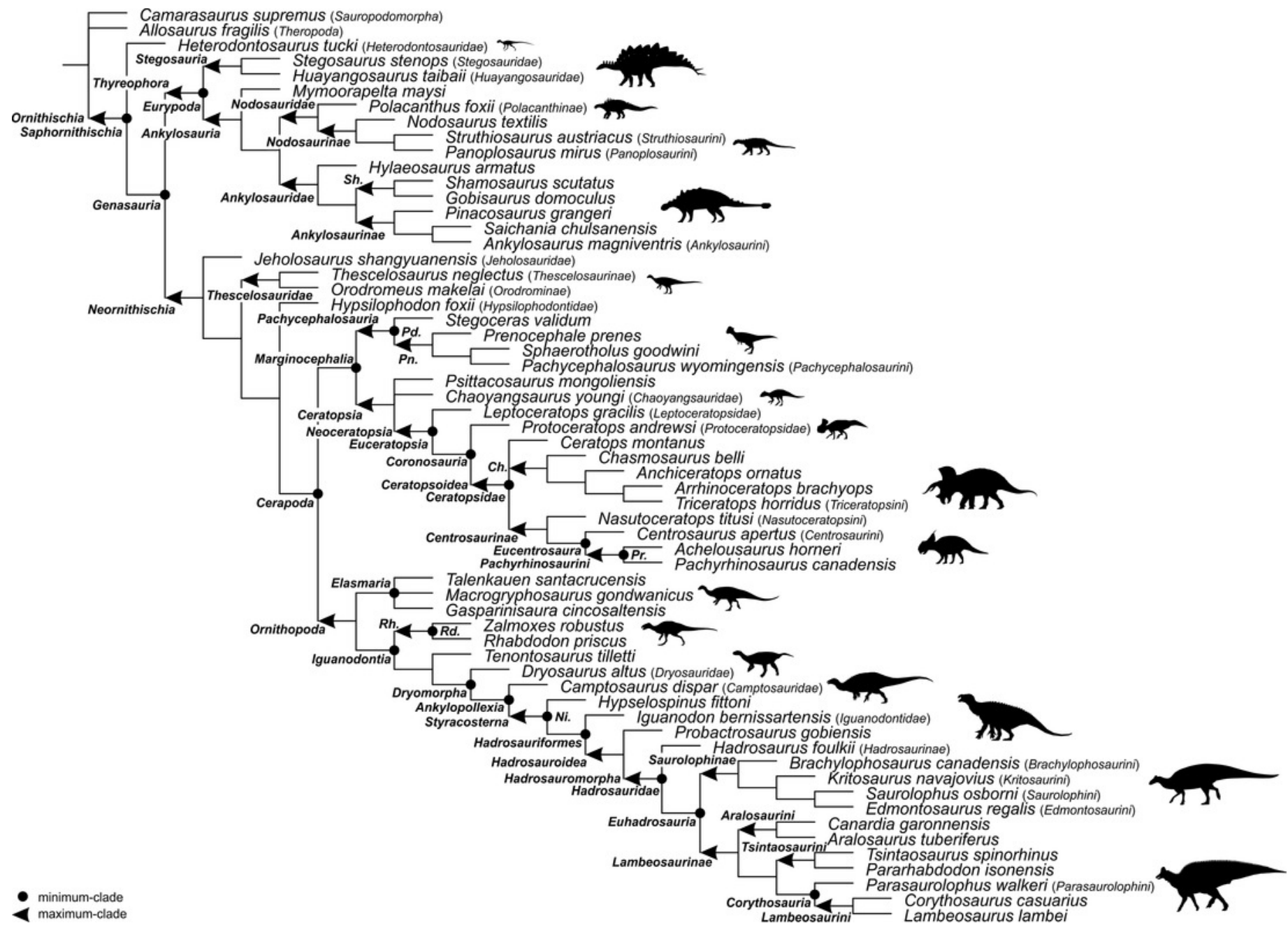




\section{Figure 2}

The phylogenetic nomenclature of ornithischian dinosaurs using the topology of Madzia et al. (2018: Fig. 4B).

Note that Nanosaurus agilis has been analyzed by Madzia et al. (2018) as 'Othnielosaurus'. The name was changed here following Carpenter \& Galton (2018). Additionally, the name Marasuchus lilloensis was placed in quotation marks to highlight that the taxon may not be distinct from Lagosuchus talampayensis (Agnolin \& Ezcurra, 2019). 


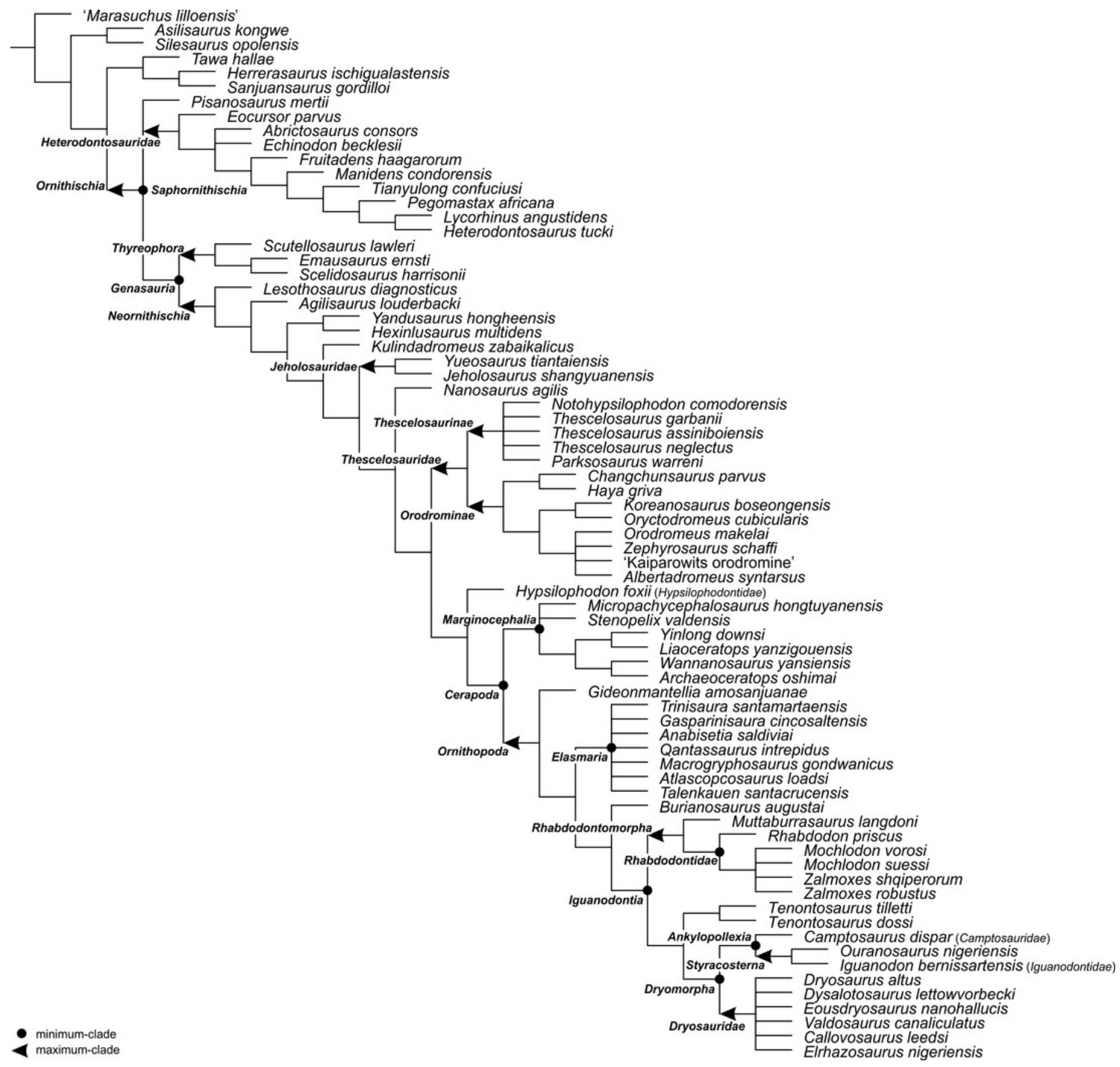




\section{Figure 3}

The phylogenetic nomenclature of ornithischian dinosaurs using the topology of Dieudonné et al. (2020: Figs 1 and 2).

Note that Dieudonné et al. (2020) followed Carpenter \& Lamanna (2015) in placing aphanoecetes within Camptosaurus. Owing to the results of recent phylogenetic analyses (e.g., Madzia et al., 2020; Verdú et al., 2020), aphanoecetes is placed here within Uteodon McDonald, 2011. Additionally, the name Psittacosaurus major was changed to Psittacosaurus lujiatunensis (following Hedrick \& Dodson, 2013), and Ankylosauria and Stegosauria of Dieudonné et al. (2020) were placed in quotation marks to highlight that these names have not been necessarily used by the authors as defined in the present study. Note also that the extent of Ornithischia is difficult to indicate on the tree because Chilesaurus diegosuarezi may represent a theropod (see Discussion). Abbreviation: An. - Ankylosauria. 
Herrerasaurus ischigualastensis

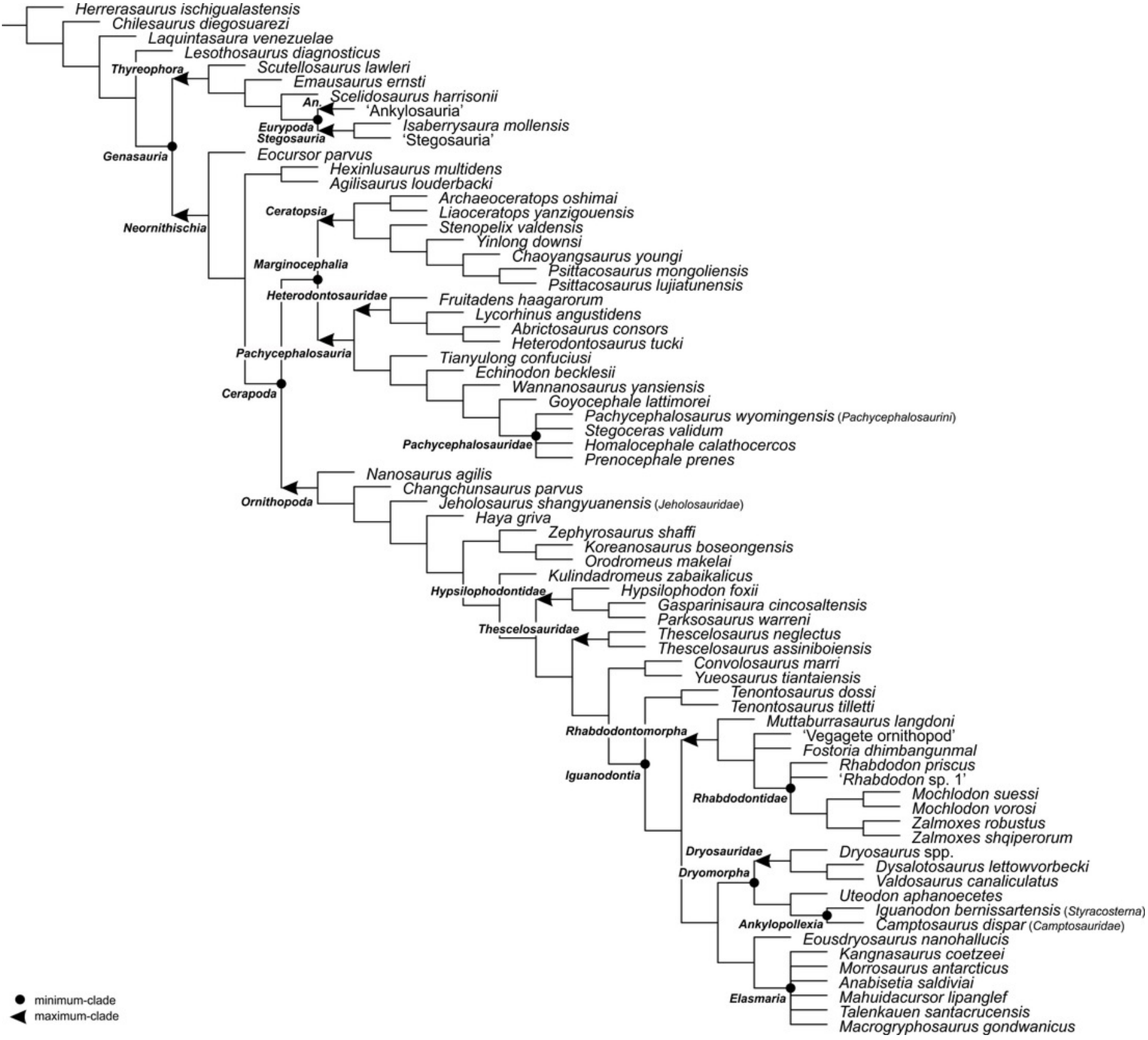




\section{Figure 4}

The phylogenetic nomenclature of ornithischian dinosaurs using the topology of Yang et al. (2020: Fig. 12).

Ankylosauria and Stegosauria of Yang et al. (2020) were placed in quotation marks to highlight that these names have not been necessarily used by the authors as defined in the present study. In turn, Psittacosauridae of Yang et al. (2020) was placed in quotation marks because the name has not been formally defined yet. Abbreviation: St. - Stegosauria. 


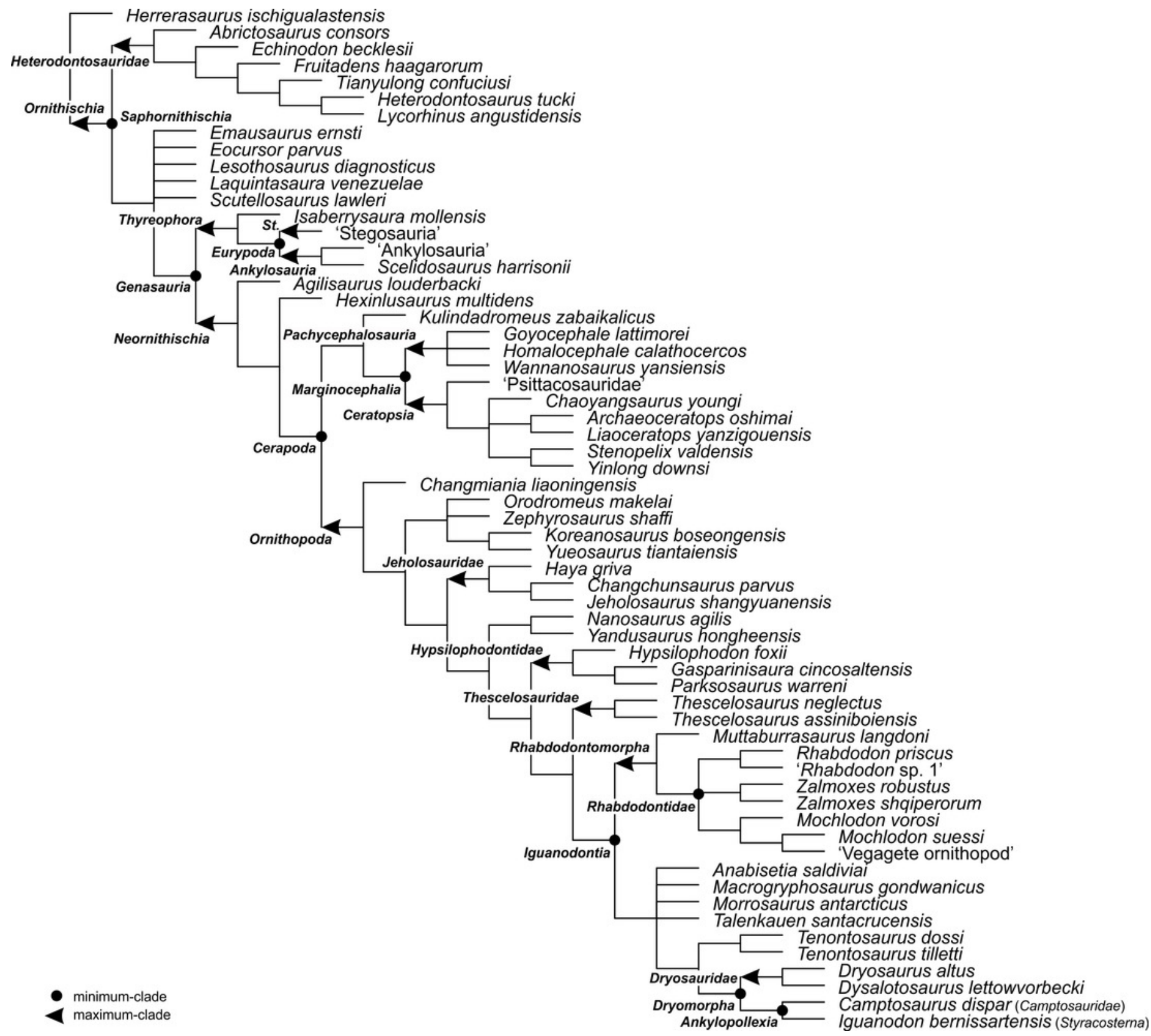


Figure 5

Specifier-based phylogeny of Hadrosauridae showing alternative placements of Hadrosaurus foulkii.

The silhouette of Lambeosaurinae was obtained from phylopic.org (Dmitry Bogdanov, CC BY 3.0). The silhouette of Hadrosaurinae/Saurolophinae was prepared by Victoria M. Arbour (CC BY 4.0).
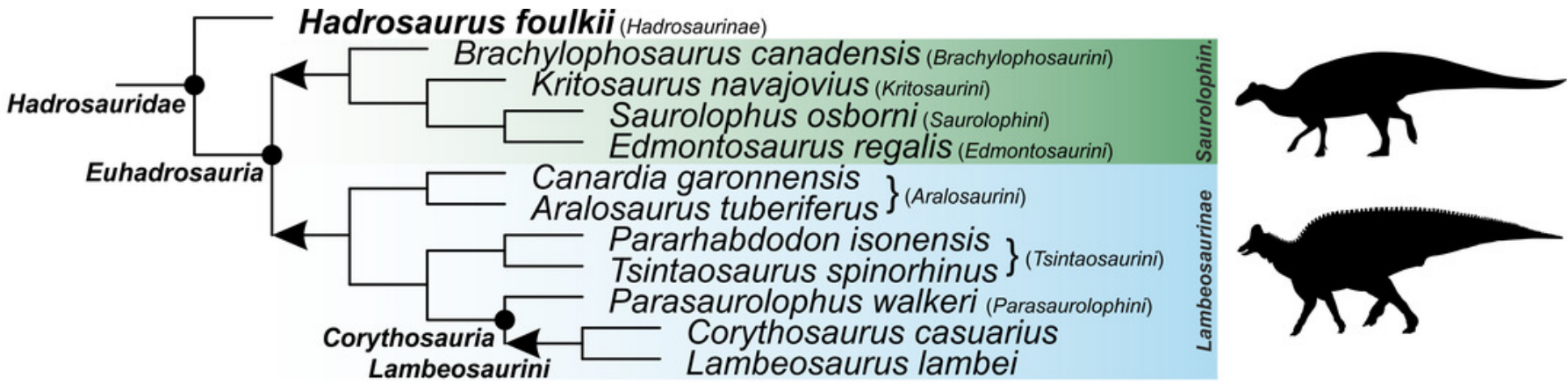

Hadrosaurus foulkii

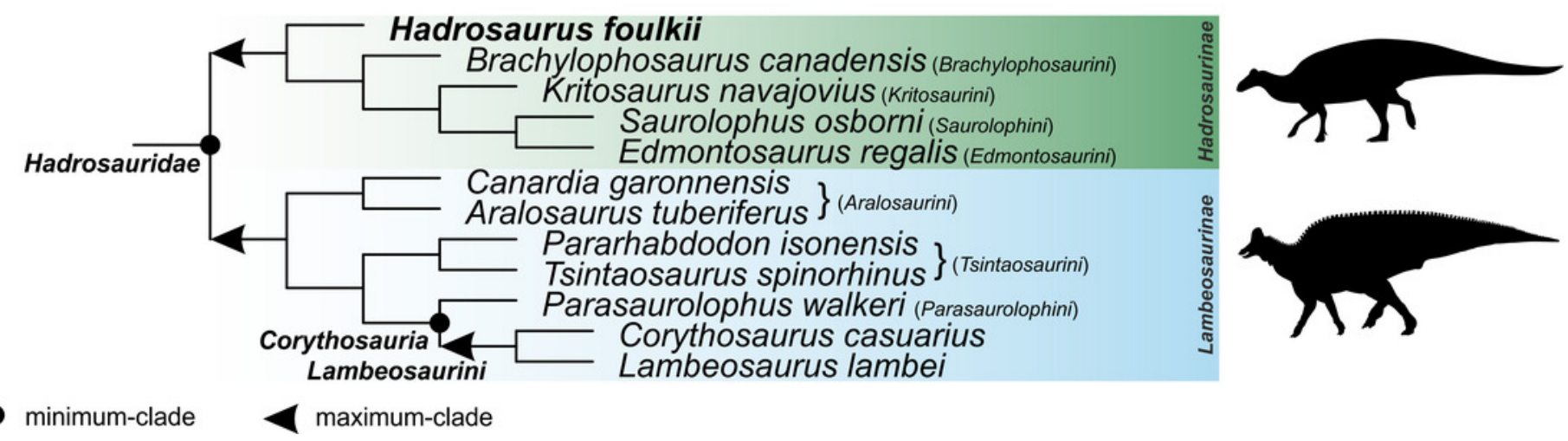




\section{Table $\mathbf{1}$ (on next page)}

The phylogenetic nomenclature of ornithischian dinosaurs. 


\begin{tabular}{|c|c|c|c|c|}
\hline Clade name & Authorship & Definition type & Abbreviated definition & Primary reference phylogeny \\
\hline Ankylopollexia & Sereno, 1986 & minimum-clade & $\begin{array}{l}\min \nabla \text { (Camptosaurus dispar }[\text { Marsh, } 1879] \& \\
\text { Iguanodon bernissartensis Boulenger in Beneden, } \\
\text { 1881) }\end{array}$ & $\begin{array}{l}\text { Figure } 12 \text { of Madzia et al. } \\
(2020)\end{array}$ \\
\hline Ankylosauria & Osborn, 1923 & maximum-clade & $\begin{array}{l}\max \nabla \text { (Ankylosaurus magniventris Brown, } 1908 \sim \\
\text { Stegosaurus stenops Marsh, 1887) }\end{array}$ & $\begin{array}{l}\text { Figure } 11 \text { of Arbour \& Currie } \\
\text { (2016) }\end{array}$ \\
\hline Ankylosauridae & Brown, 1908 & maximum-clade & $\begin{array}{l}\max \nabla \text { (Ankylosaurus magniventris Brown, } 1908 \sim \\
\text { Nodosaurus textilis Marsh, 1889) }\end{array}$ & $\begin{array}{l}\text { Figure } 11 \text { of Arbour \& Currie } \\
\text { (2016) }\end{array}$ \\
\hline Ankylosaurinae & Nopcsa, 1918 & maximum-clade & $\begin{array}{l}\max \nabla \text { (Ankylosaurus magniventris Brown, } 1908 \sim \\
\text { Shamosaurus scutatus Tumanova, 1983) }\end{array}$ & $\begin{array}{l}\text { Figure } 11 \text { of Arbour and } \\
\text { Currie (2016) }\end{array}$ \\
\hline Ankylosaurini & $\begin{array}{l}\text { Arbour \& } \\
\text { Currie, } 2016\end{array}$ & maximum-clade & $\begin{array}{l}\text { max } \nabla \text { (Ankylosaurus magniventris Brown, } 1908 \sim \\
\text { Pinacosaurus grangeri Gilmore, } 1933 \text { \& Saichania } \\
\text { chulsanensis Maryańska 1977) }\end{array}$ & $\begin{array}{l}\text { Figure } 11 \text { of Arbour and } \\
\text { Currie (2016) }\end{array}$ \\
\hline Aralosaurini & $\begin{array}{l}\text { Prieto- } \\
\text { Márquez et al., } \\
2013\end{array}$ & maximum-clade & $\begin{array}{l}\text { max } \nabla \text { (Aralosaurus tuberiferus Rozhdestvensky, } 1968 \\
\text { \& Canardia garonnensis Prieto-Márquez et al., 2013 } \\
\text { Lambeosaurus lambei Parks, } 1923 \text { \& Parasaurolophus } \\
\text { walkeri Parks, } 1922 \text { \& Tsintaosaurus spinorhinus } \\
\text { Young, 1958) }\end{array}$ & $\begin{array}{l}\text { Figure } 25 \text { of Prieto-Márquez et } \\
\text { al. (2013) }\end{array}$ \\
\hline Brachylophosaurini & $\begin{array}{l}\text { Gates et al., } \\
2011\end{array}$ & maximum-clade & $\begin{array}{l}\max \nabla \text { (Brachylophosaurus canadensis Sternberg, } 1953 \\
\sim \text { Edmontosaurus regalis Lambe, } 1917 \text { \& Hadrosaurus } \\
\text { foulkii Leidy, } 1858 \text { \& Kritosaurus navajovius Brown, } \\
1910 \text { \& Saurolophus osborni Brown, 1912) }\end{array}$ & $\begin{array}{l}\text { Figure } 18 \text { of Prieto-Márquez et } \\
\text { al. (2020) }\end{array}$ \\
\hline Camptosauridae & Marsh, 1885 & maximum-clade & $\begin{array}{l}\max \nabla(\text { Camptosaurus dispar [Marsh, 1879] } \\
\text { Iguanodon bernissartensis Boulenger in Beneden, } \\
\text { 1881) }\end{array}$ & $\begin{array}{l}\text { Figure } 13 \text { of Madzia et al. } \\
(2020)\end{array}$ \\
\hline Centrosaurinae & Lambe, 1915 & maximum-clade & $\begin{array}{l}\max \nabla(\text { Centrosaurus apertus Lambe, } 1905 \sim \\
\text { Chasmosaurus belli [Lambe, 1902] \& Triceratops } \\
\text { horridus Marsh, 1889) }\end{array}$ & Figure 9 of Chiba et al. (2018) \\
\hline Centrosaurini & Ryan et al., & maximum-clade & $\max \nabla$ (Centrosaurus apertus Lambe, $1905 \sim$ & Figure 9 of Chiba et al. (2018) \\
\hline
\end{tabular}




\begin{tabular}{|c|c|c|c|c|}
\hline & 2017 & & Pachyrhinosaurus canadensis Sternberg, 1950) & \\
\hline Cerapoda & Sereno, 1986 & minimum-clade & $\begin{array}{l}\min \nabla \text { (Iguanodon bernissartensis Boulenger in } \\
\text { Beneden, 1881 \& Pachycephalosaurus wyomingensis } \\
\text { [Gilmore, 1931] \& Triceratops horridus Marsh, 1889) }\end{array}$ & $\begin{array}{l}\text { Figure } 4 \text { of Madzia et al. } \\
\text { (2018) }\end{array}$ \\
\hline Ceratopsia & Marsh, 1890 & maximum-clade & $\begin{array}{l}\max \nabla(\text { Ceratops montanus Marsh, } 1888 \& \text { Triceratops } \\
\text { horridus Marsh, } 1889 \sim \text { Pachycephalosaurus } \\
\text { wyomingensis }[\text { Gilmore, 1931]) }\end{array}$ & $\begin{array}{l}\text { Figure } 10 \text { of Morschhauser et } \\
\text { al. (2019) }\end{array}$ \\
\hline Ceratopsidae & Marsh, 1888 & minimum-clade & $\begin{array}{l}\min \nabla \text { (Centrosaurus apertus Lambe, } 1905 \text { \& Ceratops } \\
\text { montanus Marsh, } 1888 \text { \& Chasmosaurus belli [Lambe, } \\
\text { 1902] \& Triceratops horridus Marsh, 1889) }\end{array}$ & Figure 4 of Yu et al. (2020) \\
\hline Ceratopsoidea & Hay, 1902 & maximum-clade & $\begin{array}{l}\max \nabla \text { (Ceratops montanus Marsh, } 1888 \& \text { Triceratops } \\
\text { horridus Marsh, } 1889 \sim \text { Protoceratops andrewsi } \\
\text { Granger \& Gregory, 1923) }\end{array}$ & Figure 4 of $\mathrm{Yu}$ et al. (2020) \\
\hline Chaoyangsauridae & $\begin{array}{l}\text { Zhao et al., } \\
1999\end{array}$ & maximum-clade & $\begin{array}{l}\max \nabla \text { (Chaoyangsaurus youngi Zhao et al., } 1999 \sim \\
\text { Psittacosaurus mongoliensis Osborn, } 1923 \& \\
\text { Triceratops horridus Marsh, 1889) }\end{array}$ & $\begin{array}{l}\text { Figure } 10 \text { of Morschhauser et } \\
\text { al. (2019) }\end{array}$ \\
\hline Chasmosaurinae & Lambe, 1915 & maximum-clade & $\begin{array}{l}\max \nabla \text { (Chasmosaurus belli }[\text { Lambe, 1902] \& } \\
\text { Triceratops horridus Marsh, } 1889 \sim \text { Centrosaurus } \\
\text { apertus Lambe, 1905) }\end{array}$ & $\begin{array}{l}\text { Figure 9a of Fowler \& } \\
\text { Freedman Fowler }(2020)\end{array}$ \\
\hline Clypeodonta & Norman, 2014 & minimum-clade & $\begin{array}{l}\min \nabla \in \text { Ornithopoda (Edmontosaurus regalis Lambe, } \\
1917 \text { \& Hypsilophodon foxii Huxley, 1869) }\end{array}$ & Figure 50 of Norman (2015) \\
\hline Coronosauria & Sereno, 1986 & minimum-clade & $\begin{array}{l}\min \nabla \text { (Protoceratops andrewsi Granger \& Gregory, } \\
1923 \text { \& Triceratops horridus Marsh, 1889) }\end{array}$ & $\begin{array}{l}\text { Figure } 10 \text { of Morschhauser et } \\
\text { al. (2019) }\end{array}$ \\
\hline Corythosauria & new & minimum-clade & $\begin{array}{l}\min \nabla \text { (Corythosaurus casuarius Brown, 1914a \& } \\
\text { Lambeosaurus lambei Parks, } 1923 \text { \& Parasaurolophus } \\
\text { walkeri Parks, 1922) }\end{array}$ & $\begin{array}{l}\text { Figure } 18 \text { of Prieto-Márquez et } \\
\text { al. (2020) }\end{array}$ \\
\hline Dryomorpha & Sereno, 1986 & minimum-clade & $\begin{array}{l}\min \nabla(\text { Dryosaurus altus [Marsh, 1878] \& Iguanodon } \\
\text { bernissartensis Boulenger in Beneden, 1881) }\end{array}$ & $\begin{array}{l}\text { Figure } 12 \text { of Madzia et al. } \\
(2020)\end{array}$ \\
\hline Dryosauridae & Milner \& & maximum-clade & $\max \nabla$ (Dryosaurus altus $[$ Marsh, 1878] $\sim$ Iguanodon & Figure 12 of Madzia et al. \\
\hline
\end{tabular}




\begin{tabular}{|c|c|c|c|c|}
\hline & Norman, 1984 & & bernissartensis Boulenger in Beneden, 1881) & $(2020)$ \\
\hline Edmontosaurini & Glut, 1997 & maximum-clade & $\begin{array}{l}\text { max } \nabla \text { (Edmontosaurus regalis Lambe, } 1917 \sim \\
\text { Brachylophosaurus canadensis Sternberg, } 1953 \text { \& } \\
\text { Hadrosaurus foulkii Leidy, } 1858 \text { \& Kritosaurus } \\
\text { navajovius Brown, } 1910 \text { \& Saurolophus osborni } \\
\text { Brown, 1912) }\end{array}$ & $\begin{array}{l}\text { Figure } 18 \text { of Prieto-Márquez et } \\
\text { al. (2020) }\end{array}$ \\
\hline Elasmaria & $\begin{array}{l}\text { Calvo et al., } \\
2007\end{array}$ & minimum-clade & $\begin{array}{l}\min \nabla \text { (Macrogryphosaurus gondwanicus Calvo et al., } \\
2007 \text { \& Talenkauen santacrucensis Novas et al., 2004 | } \\
\sim \text { Hypsilophodon foxii Huxley, } 1869 \text { V Iguanodon } \\
\text { bernissartensis Boulenger in Beneden, } 1881 \mathrm{~V} \\
\text { Thescelosaurus neglectus Gilmore, 1913) }\end{array}$ & $\begin{array}{l}\text { Figure } 31 \text { of Rozadilla et al. } \\
\text { (2019) }\end{array}$ \\
\hline Eucentrosaura & $\begin{array}{l}\text { Chiba et al., } \\
2018\end{array}$ & minimum-clade & $\begin{array}{l}\min \nabla \text { (Centrosaurus apertus Lambe, } 1905 \& \\
\text { Pachyrhinosaurus canadensis Sternberg, 1950) }\end{array}$ & Figure 9 of Chiba et al. (2018) \\
\hline Euceratopsia & new & minimum-clade & $\begin{array}{l}\min \nabla \text { (Leptoceratops gracilis Brown, } 1914 \mathrm{~b} \& \\
\text { Protoceratops andrewsi Granger \& Gregory, } 1923 \& \\
\text { Triceratops horridus Marsh, 1889) }\end{array}$ & Figure 4 of $\mathrm{Yu}$ et al. (2020) \\
\hline Euhadrosauria & $\begin{array}{l}\text { Weishampel et } \\
\text { al., } 1993\end{array}$ & minimum-clade & $\begin{array}{l}\min \nabla \text { (Lambeosaurus lambei Parks, } 1923 \text { \& } \\
\text { Saurolophus osborni Brown, 1912| Hadrosaurus } \\
\text { foulkii Leidy, 1858) }\end{array}$ & $\begin{array}{l}\text { Figure } 18 \text { of Prieto-Márquez et } \\
\text { al. (2020) }\end{array}$ \\
\hline Euiguanodontia & $\begin{array}{l}\text { Coria \& } \\
\text { Salgado, } 1996\end{array}$ & minimum-clade & $\begin{array}{l}\min \nabla \text { (Camptosaurus dispar [Marsh, 1879] \& } \\
\text { Dryosaurus altus }[\text { Marsh, 1878] \& Gasparinisaura } \\
\text { cincosaltensis Coria \& Salgado, 1996| } \sim \\
\text { Tenontosaurus tilletti Ostrom, 1970) }\end{array}$ & $\begin{array}{l}\text { Figure } 13 \text { of Coria \& Salgado } \\
\text { (1996) }\end{array}$ \\
\hline Euornithopoda & Sereno, 1986 & maximum-clade & $\begin{array}{l}\text { max } \nabla \in \text { Ornithopoda (Iguanodon bernissartensis } \\
\text { Boulenger in Beneden, } 1881 \sim \text { Heterodontosaurus } \\
\text { tucki Crompton \& Charig, 1962) }\end{array}$ & Figure 1 of Sereno (1999) \\
\hline Eurypoda & Sereno, 1986 & minimum-clade & $\begin{array}{l}\min \nabla \text { (Ankylosaurus magniventris Brown, } 1908 \text { \& } \\
\text { Stegosaurus stenops Marsh, 1887) }\end{array}$ & $\begin{array}{l}\text { Figure } 3 \text { of Thompson et al. } \\
\text { (2012) }\end{array}$ \\
\hline Genasauria & Sereno, 1986 & minimum-clade & $\begin{array}{l}\text { min } \nabla \text { (Ankylosaurus magniventris Brown, } 1908 \& \\
\text { Iguanodon bernissartensis Boulenger in Beneden, } 1881\end{array}$ & Figure 16 of Han et al. (2018) \\
\hline
\end{tabular}




\begin{tabular}{|c|c|c|c|c|}
\hline & & & $\begin{array}{l}\text { \& Stegosaurus stenops Marsh, } 1887 \text { \& Triceratops } \\
\text { horridus Marsh, 1889) }\end{array}$ & \\
\hline Hadrosauridae & Cope, 1869 & minimum-clade & $\begin{array}{l}\min \nabla \text { (Hadrosaurus foulkii Leidy, } 1858 \text { \& } \\
\text { Lambeosaurus lambei Parks, } 1923 \text { \& Saurolophus } \\
\text { osborni Brown, 1912) }\end{array}$ & $\begin{array}{l}\text { Figure } 18 \text { of Prieto-Márquez et } \\
\text { al. (2020) }\end{array}$ \\
\hline Hadrosauriformes & Sereno, 1997 & minimum-clade & $\begin{array}{l}\min \nabla \text { (Hadrosaurus foulkii Leidy, } 1858 \text { \& Iguanodon } \\
\text { bernissartensis Boulenger in Beneden, 1881) }\end{array}$ & $\begin{array}{l}\text { Figure } 12 \text { of Madzia et al. } \\
(2020)\end{array}$ \\
\hline Hadrosaurinae & Lambe, 1918 & maximum-clade & $\begin{array}{l}\max \nabla \text { (Hadrosaurus foulkii Leidy, } 1858 \sim \\
\text { Lambeosaurus lambei Parks, 1923) }\end{array}$ & $\begin{array}{l}\text { Figure } 5 \text { of Kobayashi et al. } \\
\text { (2019) }\end{array}$ \\
\hline Hadrosauroidea & $\begin{array}{l}\text { von Huene, } \\
1952\end{array}$ & maximum-clade & $\begin{array}{l}\max \nabla \text { (Hadrosaurus foulkii Leidy, } 1858 \sim \text { Iguanodon } \\
\text { bernissartensis Boulenger in Beneden, 1881) }\end{array}$ & $\begin{array}{l}\text { Figure } 12 \text { of Madzia et al. } \\
(2020)\end{array}$ \\
\hline Hadrosauromorpha & Norman, 2014 & maximum-clade & $\begin{array}{l}\max \nabla \text { (Hadrosaurus foulkii Leidy, } 1858 \sim \\
\text { Probactrosaurus gobiensis Rozhdestvensky, 1966) }\end{array}$ & $\begin{array}{l}\text { Figure } 12 \text { of Madzia et al. } \\
(2020)\end{array}$ \\
\hline Heterodontosauridae & Kuhn, 1966 & maximum-clade & $\begin{array}{l}\text { max } \nabla \text { (Heterodontosaurus tucki Crompton \& Charig, } \\
1962 \sim \text { Iguanodon bernissartensis Boulenger in } \\
\text { Beneden, 1881 \& Pachycephalosaurus wyomingensis } \\
\text { [Gilmore, 1931] \& Stegosaurus stenops Marsh, } 1887 \text { \& } \\
\text { Triceratops horridus Marsh, 1889) }\end{array}$ & $\begin{array}{l}\text { Figure } 4 \text { of Madzia et al. } \\
(2018)\end{array}$ \\
\hline Huayangosauridae & $\begin{array}{l}\text { Dong et al., } \\
1982\end{array}$ & maximum-clade & $\begin{array}{l}\max \nabla \text { (Huayangosaurus taibaii Dong et al., } 1982 \sim \\
\text { Stegosaurus stenops Marsh, 1887) }\end{array}$ & $\begin{array}{l}\text { Figure } 12 \text { of Maidment et al. } \\
(2020)\end{array}$ \\
\hline Hypsilophodontia & Cooper, 1985 & minimum-clade & $\begin{array}{l}\min \nabla \in \text { Ornithopoda (Hypsilophodon foxii Huxley, } \\
1869 \text { \& Tenontosaurus tilletti Ostrom, } 1970 \mid \sim \\
\text { Iguanodon bernissartensis Boulenger in Beneden, } \\
\text { 1881) }\end{array}$ & Figure 50 of Norman (2015) \\
\hline Hypsilophodontidae & Dollo, 1882 & maximum-clade & $\begin{array}{l}\max \nabla \text { (Hypsilophodon foxii Huxley, } 1869 \sim \\
\text { Iguanodon bernissartensis Boulenger in Beneden, } 1881 \\
\& \text { Rhabdodon priscus Matheron, 1869) }\end{array}$ & $\begin{array}{l}\text { Figure } 2 \text { of Dieudonné et al. } \\
(2020)\end{array}$ \\
\hline Iguanodontia & Baur, 1891 & minimum-clade & $\begin{array}{l}\min \nabla \text { (Dryosaurus altus [Marsh, 1878] \& Iguanodon } \\
\text { bernissartensis Boulenger in Beneden, } 1881 \text { \& } \\
\text { Rhabdodon priscus Matheron, } 1869 \text { \& Tenontosaurus }\end{array}$ & $\begin{array}{l}\text { Figure } 12 \text { of Madzia et al. } \\
(2020)\end{array}$ \\
\hline
\end{tabular}




\begin{tabular}{|c|c|c|c|c|}
\hline & & & $\begin{array}{l}\text { tilletti Ostrom, } 1970 \mid \sim \text { Hypsilophodon foxii Huxley, } \\
\text { 1869) }\end{array}$ & \\
\hline Iguanodontidae & $\begin{array}{l}\text { Bonaparte, } \\
1850\end{array}$ & maximum-clade & $\begin{array}{l}\max \nabla \text { (Iguanodon bernissartensis Boulenger in } \\
\text { Beneden, } 1881 \sim \text { Hadrosaurus foulkii Leidy, 1858) }\end{array}$ & $\begin{array}{l}\text { Figure } 13 \text { of Madzia et al. } \\
(2020)\end{array}$ \\
\hline Jeholosauridae & $\begin{array}{l}\text { Han et al., } \\
2012\end{array}$ & maximum-clade & $\begin{array}{l}\max \nabla \notin \text { Hypsilophodontidae } \vee \text { Thescelosauridae } \\
\text { (Jeholosaurus shangyuanensis Xu et al., 2000 } \\
\text { Hypsilophodon foxii Huxley, } 1869 \text { \& Iguanodon } \\
\text { bernissartensis Boulenger in Beneden, } 1881 \& \\
\text { Pachycephalosaurus wyomingensis [Gilmore, 1931] \& } \\
\text { Thescelosaurus neglectus Gilmore, } 1913 \& \text { Triceratops } \\
\text { horridus Marsh, 1889) }\end{array}$ & $\begin{array}{l}\text { Figure } 25 \text { of Herne et al. } \\
(2019)\end{array}$ \\
\hline Kritosaurini & Glut, 1997 & maximum-clade & $\begin{array}{l}\max \nabla \text { (Kritosaurus navajovius Brown, } 1910 \sim \\
\text { Brachylophosaurus canadensis Sternberg, } 1953 \text { \& } \\
\text { Edmontosaurus regalis Lambe, } 1917 \text { \& Hadrosaurus } \\
\text { foulkii Leidy, } 1858 \text { \& Saurolophus osborni Brown, } \\
\text { 1912) }\end{array}$ & $\begin{array}{l}\text { Figure } 18 \text { of Prieto-Márquez et } \\
\text { al. (2020) }\end{array}$ \\
\hline Lambeosaurinae & Parks, 1923 & maximum-clade & $\begin{array}{l}\max \nabla \text { (Lambeosaurus lambei Parks, } 1923 \sim \\
\text { Hadrosaurus foulkii Leidy, } 1858 \text { \& Saurolophus } \\
\text { osborni Brown, 1912) }\end{array}$ & $\begin{array}{l}\text { Figure } 18 \text { of Prieto-Márquez et } \\
\text { al. }(2020)\end{array}$ \\
\hline Lambeosaurini & $\begin{array}{l}\text { Sullivan et al., } \\
2011\end{array}$ & maximum-clade & $\begin{array}{l}\max \nabla \text { (Lambeosaurus lambei Parks, } 1923 \sim \\
\text { Aralosaurus tuberiferus Rozhdestvensky, } 1968 \text { \& } \\
\text { Parasaurolophus walkeri Parks, } 1922 \text { \& Tsintaosaurus } \\
\text { spinorhinus Young, 1958) }\end{array}$ & $\begin{array}{l}\text { Figure } 18 \text { of Prieto-Márquez et } \\
\text { al. }(2020)\end{array}$ \\
\hline Leptoceratopsidae & Nopcsa, 1923 & maximum-clade & $\begin{array}{l}\max \nabla \text { (Leptoceratops gracilis Brown, 1914b } \\
\text { Protoceratops andrewsi Granger \& Gregory, } 1923 \& \\
\text { Triceratops horridus Marsh, 1889) }\end{array}$ & $\begin{array}{l}\text { Figure } 10 \text { of Morschhauser et } \\
\text { al. (2019) }\end{array}$ \\
\hline Marginocephalia & Sereno, 1986 & minimum-clade & $\begin{array}{l}\min \nabla \text { (Ceratops montanus Marsh, } 1888 \& \\
\text { Pachycephalosaurus wyomingensis [Gilmore, 1931] \& } \\
\text { Triceratops horridus Marsh, 1889) }\end{array}$ & Figure 16 of Han et al. (2018) \\
\hline Nasutoceratopsini & $\begin{array}{l}\text { Ryan et al., } \\
2017\end{array}$ & maximum-clade & $\begin{array}{l}\max \nabla \text { (Nasutoceratops titusi Sampson et al., } 2013 \sim \\
\text { Centrosaurus apertus Lambe, 1905) }\end{array}$ & Figure 9 of Chiba et al. (2018) \\
\hline
\end{tabular}




\begin{tabular}{|c|c|c|c|c|}
\hline Neoceratopsia & Sereno, 1986 & maximum-clade & $\begin{array}{l}\max \nabla \text { (Triceratops horridus Marsh, } 1889 \sim \\
\text { Chaoyangsaurus youngi Zhao et al., } 1999 \& \text { } \\
\text { Psittacosaurus mongoliensis Osborn, 1923) }\end{array}$ & $\begin{array}{l}\text { Figure } 10 \text { of Morschhauser et } \\
\text { al. (2019) }\end{array}$ \\
\hline Neoiguanodontia & Norman, 2014 & minimum-clade & $\begin{array}{l}\min \nabla \text { (Hypselospinus fittoni }[\text { Lydekker, 1889] \& } \\
\text { Iguanodon bernissartensis Boulenger in Beneden, } 1881 \\
\text { \& Parasaurolophus walkeri Parks 1922) }\end{array}$ & Figure 2.26 of Norman (2014) \\
\hline Neornithischia & Cooper, 1985 & maximum-clade & $\begin{array}{l}\max \nabla \text { (Iguanodon bernissartensis Boulenger in } \\
\text { Beneden, } 1881 \text { \& Triceratops horridus Marsh, } 1889 \sim \\
\text { Ankylosaurus magniventris Brown, } 1908 \& \\
\text { Stegosaurus stenops Marsh, 1887) }\end{array}$ & $\begin{array}{l}\text { Figure } 4 \text { of Madzia et al. } \\
\text { (2018) }\end{array}$ \\
\hline Nodosauridae & Marsh, 1890 & maximum-clade & $\begin{array}{l}\max \nabla \text { (Nodosaurus textilis Marsh, } 1889 \sim \\
\text { Ankylosaurus magniventris Brown, 1908) }\end{array}$ & $\begin{array}{l}\text { Figure } 5 \text { of Rivera-Sylva et al. } \\
\text { (2018a) }\end{array}$ \\
\hline Nodosaurinae & Abel, 1919 & maximum-clade & $\begin{array}{l}\max \nabla \text { (Nodosaurus textilis Marsh, } 1889 \sim \\
\text { Hylaeosaurus armatus Mantell, } 1833 \text { \& Mymoorapelta } \\
\text { maysi Kirkland \& Carpenter, } 1994 \text { \& Polacanthus foxii } \\
\text { Owen in Anonymous, 1865) }\end{array}$ & $\begin{array}{l}\text { Figure } 5 \text { of Rivera-Sylva et al. } \\
\text { (2018a) }\end{array}$ \\
\hline Ornithischia & Seeley, 1888 & maximum-clade & $\begin{array}{l}\max \nabla \text { (Iguanodon bernissartensis Boulenger in } \\
\text { Beneden, } 1881 \sim \text { Allosaurus fragilis Marsh, 1877a \& } \\
\text { Camarasaurus supremus Cope, } 1877 \text { ) }\end{array}$ & $\begin{array}{l}\text { Figure } 4 \text { of Madzia et al. } \\
\text { (2018) }\end{array}$ \\
\hline Ornithopoda & Marsh, 1881 & maximum-clade & $\begin{array}{l}\max \nabla \text { (Iguanodon bernissartensis Boulenger in } \\
\text { Beneden, 1881 } \sim \text { Pachycephalosaurus wyomingensis } \\
\text { [Gilmore, 1931] \& Triceratops horridus Marsh, 1889) }\end{array}$ & $\begin{array}{l}\text { Figure } 4 \text { of Madzia et al. } \\
\text { (2018) }\end{array}$ \\
\hline Orodrominae & $\begin{array}{l}\text { Brown et al., } \\
2013\end{array}$ & maximum-clade & $\begin{array}{l}\max \nabla \in \text { Hypsilophodontidae } \vee \text { Thescelosauridae } \\
\text { (Orodromeus makelai Horner \& Weishampel, } 1988 \sim \\
\text { Hypsilophodon foxii Huxley, } 1869 \text { \& Thescelosaurus } \\
\text { neglectus Gilmore, 1913) }\end{array}$ & $\begin{array}{l}\text { Figure } 4 \text { of Madzia et al. } \\
\text { (2018) }\end{array}$ \\
\hline Pachycephalosauria & $\begin{array}{l}\text { Maryańska \& } \\
\text { Osmólska, } \\
1974\end{array}$ & maximum-clade & $\begin{array}{l}\max \nabla \text { (Pachycephalosaurus wyomingensis [Gilmore, } \\
\text { 1931] Ceratops montanus Marsh, } 1888 \& \\
\text { Triceratops horridus Marsh, 1889) }\end{array}$ & $\begin{array}{l}\text { Figure } 27 \text { of Schott \& Evans } \\
\text { (2017) }\end{array}$ \\
\hline
\end{tabular}




\begin{tabular}{|c|c|c|c|c|}
\hline Pachycephalosauridae & $\begin{array}{l}\text { Sternberg, } \\
1945\end{array}$ & minimum-clade & $\begin{array}{l}\min \nabla \text { (Pachycephalosaurus wyomingensis [Gilmore, } \\
\text { 1931] \& Stegoceras validum Lambe, } 1902 \mid \sim \\
\text { Heterodontosaurus tucki Crompton \& Charig, 1962) }\end{array}$ & $\begin{array}{l}\text { Figure } 27 \text { of Schott \& Evans } \\
(2017)\end{array}$ \\
\hline Pachycephalosaurinae & Sereno, 1997 & maximum-clade & $\begin{array}{l}\max \nabla \text { (Pachycephalosaurus wyomingensis [Gilmore, } \\
\text { 1931] Stegoceras validum Lambe, 1902) }\end{array}$ & $\begin{array}{l}\text { Figure } 27 \text { of Schott \& Evans } \\
\text { (2017) }\end{array}$ \\
\hline Pachycephalosaurini & Sullivan, 2003 & maximum-clade & $\begin{array}{l}\max \nabla \text { (Pachycephalosaurus wyomingensis [Gilmore, } \\
\text { 1931] Prenocephale prenes Maryańska \& Osmólska, } \\
1974 \text { \& Sphaerotholus goodwini Williamson \& Carr, } \\
\text { 2003) }\end{array}$ & $\begin{array}{l}\text { Figure } 27 \text { of Schott \& Evans } \\
\text { (2017) }\end{array}$ \\
\hline Pachyrhinosaurini & $\begin{array}{l}\text { Fiorillo \& } \\
\text { Tykoski, } 2012\end{array}$ & maximum-clade & $\begin{array}{l}\max \nabla \text { (Pachyrhinosaurus canadensis Sternberg, } 1950 \\
\sim \text { Centrosaurus apertus Lambe, 1905) }\end{array}$ & Figure 9 of Chiba et al. (2018) \\
\hline Pachyrostra & $\begin{array}{l}\text { Fiorillo \& } \\
\text { Tykoski, } 2012\end{array}$ & minimum-clade & $\begin{array}{l}\min \nabla \text { (Achelousaurus horneri Sampson, } 1995 \& \\
\text { Pachyrhinosaurus canadensis Sternberg, 1950) }\end{array}$ & Figure 9 of Chiba et al. (2018) \\
\hline Panoplosaurini & new & maximum-clade & $\begin{array}{l}\max \nabla \text { (Panoplosaurus mirus Lambe, } 1919 \sim \\
\text { Nodosaurus textilis Marsh, } 1889 \text { \& Struthiosaurus } \\
\text { austriacus Bunzel, 1871) }\end{array}$ & $\begin{array}{l}\text { Figure } 5 \text { of Rivera-Sylva et al. } \\
\text { (2018a) }\end{array}$ \\
\hline Parasaurolophini & Glut, 1997 & maximum-clade & $\begin{array}{l}\max \nabla \text { (Parasaurolophus walkeri Parks, } 1922 \sim \\
\text { Aralosaurus tuberiferus Rozhdestvensky, } 1968 \text { \& } \\
\text { Lambeosaurus lambei Parks, } 1923 \text { \& Tsintaosaurus } \\
\text { spinorhinus Young, 1958) }\end{array}$ & $\begin{array}{l}\text { Figure } 18 \text { of Prieto-Márquez et } \\
\text { al. (2020) }\end{array}$ \\
\hline Polacanthinae & $\begin{array}{l}\text { Lapparent \& } \\
\text { Lavocat, } 1955\end{array}$ & maximum-clade & $\begin{array}{l}\max \nabla \in \text { Ankylosauridae } \vee \text { Nodosauridae (Polacanthus } \\
\text { foxii Owen in Anonymous, } 1865 \sim \text { Ankylosaurus } \\
\text { magniventris Brown, } 1908 \text { \& Nodosaurus textilis } \\
\text { Marsh, 1889) }\end{array}$ & Figure 9 of Yang et al. (2013) \\
\hline Protoceratopsidae & $\begin{array}{l}\text { Granger \& } \\
\text { Gregory, } 1923\end{array}$ & maximum-clade & $\begin{array}{l}\max \nabla \text { (Protoceratops andrewsi Granger \& Gregory, } \\
1923 \sim \text { Ceratops montanus Marsh, } 1888 \text { \& } \\
\text { Leptoceratops gracilis Brown, } 1914 \mathrm{~b} \text { \& Triceratops } \\
\text { horridus Marsh, 1889) }\end{array}$ & $\begin{array}{l}\text { Figure } 10 \text { of Morschhauser et } \\
\text { al. (2019) }\end{array}$ \\
\hline
\end{tabular}




\begin{tabular}{|c|c|c|c|c|}
\hline Rhabdodontidae & $\begin{array}{l}\text { Weishampel et } \\
\text { al., } 2003\end{array}$ & minimum-clade & $\begin{array}{l}\min \nabla(\text { Rhabdodon priscus Matheron, } 1869 \& \\
\text { Zalmoxes robustus [Nopcsa, 1900]) }\end{array}$ & $\begin{array}{l}\text { Figure } 4 \text { of Madzia et al. } \\
(2018)\end{array}$ \\
\hline Rhabdodontomorpha & $\begin{array}{l}\text { Dieudonné et } \\
\text { al., } 2016\end{array}$ & maximum-clade & $\begin{array}{l}\max \nabla \text { (Rhabdodon priscus Matheron, } 1869 \sim \\
\text { Hypsilophodon foxii Huxley, } 1869 \text { \& Iguanodon } \\
\text { bernissartensis Boulenger in Beneden, 1881) }\end{array}$ & $\begin{array}{l}\text { Figure } 2 \text { of Dieudonné et al. } \\
(2020)\end{array}$ \\
\hline Saphornithischia & new & minimum-clade & $\begin{array}{l}\min \nabla \text { (Heterodontosaurus tucki Crompton \& Charig, } \\
1962 \text { \& Iguanodon bernissartensis Boulenger in } \\
\text { Beneden, } 1881 \text { \& Stegosaurus stenops Marsh, } 1887 \text { \& } \\
\text { Triceratops horridus Marsh, 1889) }\end{array}$ & $\begin{array}{l}\text { Figure } 4 \text { of Madzia et al. } \\
\text { (2018) }\end{array}$ \\
\hline Saurolophinae & Brown, 1914a & maximum-clade & $\begin{array}{l}\max \nabla \text { (Saurolophus osborni Brown, } 1912 \sim \\
\text { Lambeosaurus lambei Parks, } 1923 \mid \sim \text { Hadrosaurus } \\
\text { foulkii Leidy, 1858) }\end{array}$ & $\begin{array}{l}\text { Figure } 18 \text { of Prieto-Márquez et } \\
\text { al. (2020) }\end{array}$ \\
\hline Saurolophini & Glut, 1997 & maximum-clade & $\begin{array}{l}\max \nabla \text { (Saurolophus osborni Brown, } 1912 \sim \\
\text { Brachylophosaurus canadensis Sternberg, } 1953 \text { \& } \\
\text { Edmontosaurus regalis Lambe, } 1917 \text { \& Hadrosaurus } \\
\text { foulkii Leidy, } 1858 \text { \& Kritosaurus navajovius Brown, } \\
\text { 1910) }\end{array}$ & $\begin{array}{l}\text { Figure } 18 \text { of Prieto-Márquez et } \\
\text { al. (2020) }\end{array}$ \\
\hline Shamosaurinae & $\begin{array}{l}\text { Tumanova, } \\
1983\end{array}$ & maximum-clade & $\begin{array}{l}\text { max } \nabla \text { (Gobisaurus domoculus Vickaryous et al., } 2001 \\
\text { \& Shamosaurus scutatus Tumanova, 1983 } \\
\text { Ankylosaurus magniventris Brown, 1908) }\end{array}$ & $\begin{array}{l}\text { Figure } 11 \text { of Arbour \& Currie } \\
\text { (2016) }\end{array}$ \\
\hline Stegosauria & Marsh, 1877b & maximum-clade & $\begin{array}{l}\max \nabla \text { (Stegosaurus stenops Marsh, } 1887 \sim \\
\text { Ankylosaurus magniventris Brown, 1908) }\end{array}$ & $\begin{array}{l}\text { Figure } 12 \text { of Maidment et al. } \\
(2020)\end{array}$ \\
\hline Stegosauridae & Marsh, 1880 & maximum-clade & $\begin{array}{l}\max \nabla \text { (Stegosaurus stenops Marsh, } 1887 \sim \\
\text { Huayangosaurus taibaii Dong et al., 1982) }\end{array}$ & $\begin{array}{l}\text { Figure } 12 \text { of Maidment et al. } \\
(2020)\end{array}$ \\
\hline Struthiosaurini & new & maximum-clade & $\begin{array}{l}\max \nabla(\text { Struthiosaurus austriacus } \text { Bunzel, } 1871 \sim \\
\text { Nodosaurus textilis } \text { Marsh, } 1889 \text { \& Panoplosaurus } \\
\text { mirus Lambe, 1919) }\end{array}$ & $\begin{array}{l}\text { Figure } 5 \text { of Rivera-Sylva et al. } \\
\text { (2018a) }\end{array}$ \\
\hline Styracosterna & Sereno, 1986 & maximum-clade & $\begin{array}{l}\max \nabla(\text { Iguanodon bernissartensis Boulenger in } \\
\text { Beneden, } 1881 \sim \text { Camptosaurus dispar [Marsh, } 1879])\end{array}$ & $\begin{array}{l}\text { Figure } 12 \text { of Madzia et al. } \\
(2020)\end{array}$ \\
\hline
\end{tabular}




\begin{tabular}{|c|c|c|c|c|}
\hline Thescelosauridae & $\begin{array}{l}\text { Sternberg, } \\
1937\end{array}$ & maximum-clade & $\begin{array}{l}\max \nabla \text { (Thescelosaurus neglectus Gilmore, } 1913 \sim \\
\text { Iguanodon bernissartensis Boulenger in Beneden, } 1881 \\
\mid \sim \text { Hypsilophodon foxii Huxley, 1869) }\end{array}$ & $\begin{array}{l}\text { Figure } 4 \text { of Madzia et al. } \\
\text { (2018) }\end{array}$ \\
\hline Thescelosaurinae & $\begin{array}{l}\text { Sternberg, } \\
1940\end{array}$ & maximum-clade & $\begin{array}{l}\max \nabla \in \text { Hypsilophodontidae } \vee \text { Thescelosauridae } \\
\text { (Thescelosaurus neglectus Gilmore, } 1913 \sim \\
\text { Hypsilophodon foxii Huxley, } 1869 \text { \& Orodromeus } \\
\text { makelai Horner \& Weishampel, 1988) }\end{array}$ & $\begin{array}{l}\text { Figure } 4 \text { of Madzia et al. } \\
\text { (2018) }\end{array}$ \\
\hline Thyreophora & Nopcsa, 1915 & maximum-clade & $\begin{array}{l}\max \nabla \text { (Ankylosaurus magniventris Brown, } 1908 \& \\
\text { Stegosaurus stenops Marsh, } 1887 \sim \text { Iguanodon } \\
\text { bernissartensis Boulenger in Beneden, } 1881 \& \\
\text { Triceratops horridus Marsh, 1889) }\end{array}$ & Figure 16 of Han et al. (2018) \\
\hline Triceratopsini & $\begin{array}{l}\text { Longrich, } \\
2011\end{array}$ & maximum-clade & $\begin{array}{l}\max \nabla \text { (Triceratops horridus Marsh, } 1889 \sim \\
\text { Anchiceratops ornatus Brown, } 1914 \mathrm{c} \& \\
\text { Arrhinoceratops brachyops Parks, 1925) }\end{array}$ & $\begin{array}{l}\text { Figure 9a of Fowler \& } \\
\text { Freedman Fowler }(2020)\end{array}$ \\
\hline Tsintaosaurini & $\begin{array}{l}\text { Prieto- } \\
\text { Márquez et al., } \\
2013\end{array}$ & maximum-clade & $\begin{array}{l}\text { max } \nabla \text { (Pararhabdodon isonensis Casanovas Cladellas } \\
\text { et al., } 1993 \text { \& Tsintaosaurus spinorhinus Young, } 1958 \\
\sim \text { Aralosaurus tuberiferus Rozhdestvensky, } 1968 \text { \& } \\
\text { Lambeosaurus lambei Parks, } 1923 \text { \& Parasaurolophus } \\
\text { walkeri Parks, 1922) }\end{array}$ & $\begin{array}{l}\text { Figure } 18 \text { of Prieto-Márquez et } \\
\text { al. }(2020)\end{array}$ \\
\hline
\end{tabular}

